\title{
ASSESSING PLANT DESIGN WITH REGARD TO MPC PERFORMANCE
}

\author{
F.A.M. Strutzel ${ }^{1}$, I. David L. Bogle ${ }^{2}$
}

Centre for Process Systems Engineering, Department of Chemical Engineering, University College London, Torrington Place, London WC1E 7JE, UK

\begin{abstract}
Model Predictive Control is ubiquitous in the chemical industry and offers great advantages over traditional controllers. Notwithstanding, new plants are being projected without taking into account how design choices affect the MPC's ability to deliver better control and optimization. Thus a methodology to determine if a certain design option favours or hinders MPC performance would be desirable. This paper presents the economic MPC optimization index whose intended use is to provide a procedure to compare different designs for a given process, assessing how well they can be controlled and optimised by a zone constrained MPC. The index quantifies the economic benefits available and how well the plant performs under MPC control given the plant's controllability properties, requirements and restrictions. The index provides a monetization measure of expected control performance.

This approach assumes the availability of a linear state-space model valid within the control zone defined by the upper and lower bounds of each controlled and manipulated variable. We have used a model derived from simulation step tests as a practical way to use the method. The impact of model uncertainty on the methodology is discussed. An analysis of the effects of disturbances on the index illustrates how they may reduce profitability by restricting the ability of a MPC to reach dynamic equilibrium near process restrictions, which in turn increases product quality giveaway and costs. A case of study consisting of four alternative designs for a realistically sized crude oil atmospheric distillation plant is provided in order to demonstrate the applicability of the index.
\end{abstract}

\footnotetext{
${ }^{1}$ On leave from Petrobras Oil Company, Presidente Bernardes Refinery (RPBC), Cubatão, São Paulo, Brazil.

${ }^{2}$ Corresponding author. Tel.: +44 207679 3803; fax: +44 2073832348.

E-mail address: d.bogle@ucl.ac.uk (I. David L. Bogle)
} 


\section{Keywords}

Integrated Process Design and Control, Model Predictive Control (MPC), Zone Constrained Model Predictive Control, Zone Control, Controllability Analysis, Crude Oil Distillation.

\section{Introduction and Motivation}

The ultimate goal of any chemical plant is to produce products profitably and thus its operation must be stable and optimised. To reach such a goal it is necessary to provide the plant with a correctly engineered control system, which must possess a convenient set of controlled and manipulated variables, clearly defined control objectives, and optimal tuning parameters. MPC control schemes are popular solutions to meet the control requirements of complex chemical processes due to their capacity for dealing with multivariable problems and inverse response, as well as time delayed and highly nonlinear systems. Assuming that the MPC is well engineered, the limitations on its ability to control and optimize chemical plants is related to the plant's own characteristics. The maximum number of controlled variables it can keep at their desired values in the face of disturbances and saturation of control elements is ultimately defined by process dynamics, in turn reflected in the plant's model. Most published works in MPC control theory have been focusing on the development of new algorithms but we believe this field has matured and larger gains may be achieved by switching the focus back to the design of the process.

Controllability and resiliency as judged by many published indices (for example the Disturbance Cost Index (Lewin (1996), Solovyev and Lewin (2002)) are required but by themselves, isolated from process economics analysis, they may be a poor guide for selecting a process design. To maximise profitability a controller's ability to return the process to the original operating region is less important than the MPC's capability to operate close to the controlled variables' restrictions, reducing quality giveaway and energy costs and therefore maximizing operating revenue, and doing this without producing off spec products or compromising safety. As can be seen from figure 1, which presents a simplified scheme to illustrate the economic benefits of MPC, the reduced variability allows the process to operate closer to restrictions, maximizing output. We present an approach that explicitly relates the control effort index to plant operational revenue. 


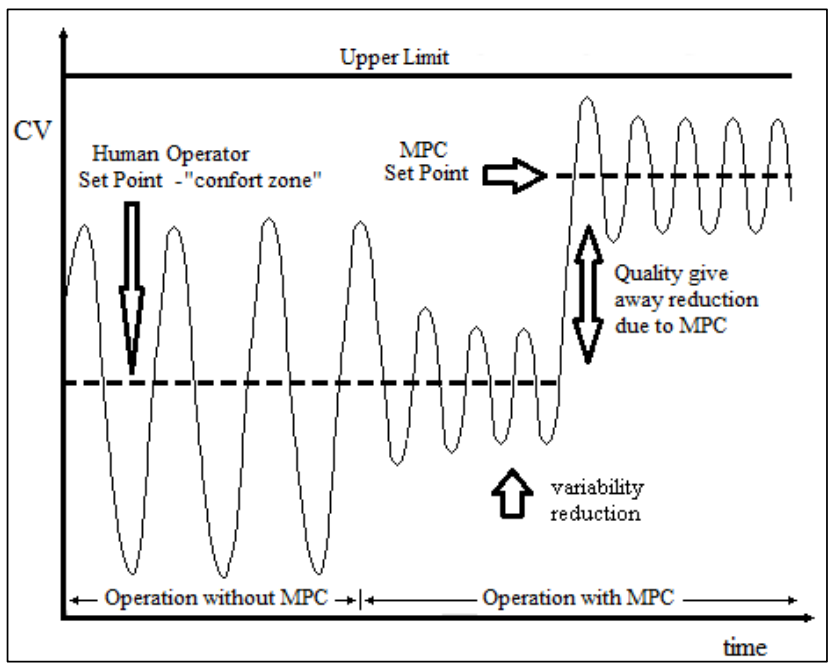

Figure $1-$ MPC reduces quality give away.

Many years of experience with MPC have shown its ability to improve performance in this way, but if we can assume this fact how should this affect the chemical design process? Given a set of controlled and manipulated variables and their bounds, what effect has a certain plant layout modification on MPC performance? Or how should changes in product specifications affect the layout if we assume MPC will be used? The present work proposes an easy new way to assess those impacts that is valid for any zone constrained MPC algorithm.

The problem being addressed in this work may be summarised as follows: within the range of all possible operating points or conditions available for a given plant, which has been defined by the MPC control zones, what is the most profitable? Is the path from an initial state to this desired state feasible or does it violate soft constraints? Can this optimal state be sustained by the plant? If we have a number of different plant designs, how does each plant's optimal operating point compare? In the current work an optimal trajectory for each plant is defined, evaluated and inspected by the control engineer who then proceeds with a comparison between the process candidate designs. The approach presented in this paper is based on the premise that disturbances are known and estimated a priori and follow a given time-dependant profile.

This paper is organized in 5 sections. Section 2 contains a brief presentation of the economic MPC optimization index. Section 3 will feature a case of study concerning the assessment of four possible layouts for a crude oil distillation unit for which the new methodology will be applied. Section 4 will present and discuss the results obtained 
through the employment of the described methodology. Section 5 shows how to consider model uncertainty and conclusions and future work are presented in section 6 .

\subsection{Integrated Process and Control Design Methodologies}

Work on the integration of design and control for chemical processes produced analysis such as those found in Mohideen, Perkins and Pistikopoulos (1996) and Perkins and Walsh (1996), where methods that possess an explicit economic component were presented for optimal plant design involving a classical feedback control structure. This involves attributing a cost to a control performance measure such as the integral error, e.g., ISE or IAE, performing a worst-case design optimization for tuning one or more PID controllers for optimal system response and then varying a number of equipment design parameters. This is repeated until the global solution is found. This avoids equipment oversizing and thus decreases costs. Among the indices developed to assess the resilience of chemical plants subject to disturbances are Skogestad and Morari (1987), who presented a method for obtaining a bound on the magnitude of the worst-case relative gain, and Weitz and Lewin (1996) who provided a procedure that relies on a modelling strategy that makes use of a linear approximation obtained from steady-state flowsheet information.

Sánchez-Sánchez and Ricardez-Sandoval, (2013b) proposed a method which allows the reduction of safety overdesign factors, avoiding an unnecessary level of conservatism that leads to expensive designs. Performing dynamic controllability analysis of the basic regulatory control loops (i.e. PID controllers) reduces the need for intentional equipment oversizing required to guarantee control stability.

A number of researchers have extended the analysis to large scale systems. Alhammadi and Romagnoli (2004) proposed an integrated plantwide framework that incorporates not only the usual metrics such as controllability and economic performance, but also environmental performance and energy integration, resulting in a multi-objective optimisation problem. The method was applied to a large-scale Vinyl Chloride Monomer plant. Bansal et al. (2000) propose an approach for the flexibility analysis and design of linear systems, based on parametric programming, and Bansal et al. (2002) generalise and unify this approach for the flexibility analysis and design of nonlinear systems. Both papers used rigorous binary distillation models as cases for study ( 2 components, 3 PI controllers and 7 subsystems). Ricardez-Sandoval et al. (2011) proposed a methodology 
used to estimate analytical bounds on the worst-case variability of disturbances and parametric model uncertainties suitable for application to large-scale systems (using the Tenessee Eastman Problem which has 8 components, 8 PI controllers and 5 subsystems). Trainor et al. (2013) presents a methodology for the optimal process and control design of large-scale systems under uncertainty that incorporates robust feasibility and stability analyses formulated as convex mathematical problems (applied to a ternary distillation problem with 2 PI controllers and 6 subsystems). Alvarado-Morales et al. (2010) presented an integrated synthesis framework and applied it to two large-scale processes: a bioethanol production plant as well as succinic acid production (with 16 components, 2 PI controllers and 7 subsystems).

While all systems addressed by works above are genuinely large-scale, oil refining processes, such as the set of crude oil distillation plants studied here, presents a particular challenge. The phenomenological models used by the papers presented in this section are adequate for separation processes of mixtures presenting near-ideal behaviour, i.e., where deviation from Raoult's law can be ignored, or mixtures of chemically similar solvents, or non-ideal solutions to which Raoult's law applies and fugacity and activity coefficients can be easily calculated. But difficulties arise when dealing with petroleum fractions: each subsystem has usually dozens of non-ideal hypothetical components; severe operating conditions mean that the behaviour of gases, solutions and mixtures is also nonideal; multiphase flow is very common and hard to model adequately; and equipment designs are intricate. The best simulators for this kind of process do not provide their set of equations, which are closed source intellectual property. For all these reasons, and the time and engineering effort required for rigorous modelling is always very large and, unless models are linearised, even with the optimization solvers and processing power available at the time of writing it is doubtful that a solution could be found in reasonable time. This paper aims to offer an alternative controllability analysis approach that is better suited for the plant-wide design of oil refining processes, and also to include the use of Model Predictive Control as main control strategy.

\subsection{Integrated Process Designs using Model Predictive Control}

Adapting this kind of methodology to deal with MPC is a very challenging task which has been only recently receiving due attention from researchers. Perhaps the first attempt to extend the classical integrated design and control approach using MPC control was carried out by Brengel and Seider (1992). Francisco et al. (2011) presented a 
methodology to provide simultaneously the plant dimensions, the control parameters and a steady state working point using an IHMPC formulation with a terminal penalty including considering model uncertainty for robustness. The optimization problem is a multi-objective nonlinear constrained optimization problem, including capital and operating costs and controllability indices.

A similar method was presented by Bahakim and Ricardez-Sandoval (2014), involving the identification of an internal MPC model and solving an optimization problem at each time step in which the MPC algorithm rejected stochastic worst-case disturbances. The control performance was added to a design cost function that also included the capital costs derived from equipment sizing parameters. The methodology considers how often the worst-case would occur and the level of significance of constraint violations, arguing that it is not reasonable to overdesign the plant with increased costs because of extremely rare situations. Chawankul et al. (2007) presented another method which attributed a variability cost for controlled variables during dynamic operation. The sum of capital and operating costs are combined into a single objective function. Process nonlinearity is represented by the use of a nominal linear model with parameter uncertainty in the MPC internal model. The worst-case variability was quantified and its associated economic cost was calculated and referred to as the robust variability cost. This approach avoided nonlinear dynamic simulations, offering computational advantages.

Sakizlis et al. (2004) presented an extension of the process and control design framework that incorporates parametric model-based predictive controllers. Applying parametric programming for the controller derivation, the authors removed the need for solving an optimization problem on-line by giving rise to a closed-form controller structure. Sánchez-Sánchez and Ricardez-Sandoval (2013a) solve layers of optimisation problems: dynamic flexibility analysis, a robust dynamic feasibility analysis, a nominal stability analysis, and a robust asymptotic stability analysis to determine the optimal design. The methodology incorporates structural decisions in the analysis for the selection of an optimal process flowsheet, while formulating the analysis as convex problem for which efficient numerical algorithms exist. Ricardez-Sandoval et al. (2009) point out that the algorithmic framework involving MPC is computationally demanding even when a small number of process units are considered. 
A different approach to access economic performance is needed to deal with the multivariable "zone control" problem addressed by most commercial MPC packages. This variation of a partial control problem is defined by the existence of "zone constraints" in which every controlled variable is bounded by maximum and minimum desired values so that the control problem is not to keep each one at a fixed set-point but instead to keep all of them bounded. Often the MPC is not able to keep all controlled variables within their control zones due to the lack of degrees of freedom, which may lead to the violation of some restrictions, which are called 'soft constraints'. Every manipulated variable also has its required maximum and minimum values, or 'hard constraints' which must never be violated. It is also a standard feature for industrial control applications to perform simultaneous process control and optimization. Several MPC packages, including Honeywell ${ }^{\text {TM }}$ MPC, Shell-Yokogawa Exa-SMOC ${ }^{\text {TM }}$, Emerson DeltaV ${ }^{\mathrm{TM}}$ Predict and AspenTech DMCplus ${ }^{\mathrm{TM}}$, offer both of these features.

Most recent academic research has been focusing on robust MPC and other schemes with guaranteed stability, which translate into slower control actions, instead of real needs and performance (as noted by Bemporad and Morari (1999) robust MPC control actions may be excessively conservative). Some examples of research concerning zone control are found in González and Odloak (2009), Grosman et al. (2010), Luo et al. (2012) and Zhang et al. (2011). Porfírio and Odloak (2011), Gouvêa and Odloak (1998) and Adetola and Guay (2010) address the integration of economic optimization and MPC control.

The work presented here aims to provide a workable solution to assess alternative designs based on control performance and its economic ramifications for highly complex chemical plants controlled by zone control MPC algorithms. The approach makes use of the most readily available models, which are usually empirical models identified from plant tests, such as step tests. Here a new controllability index is used, the economic MPC optimization index, for assessing process plants for which linear state-space models are available indicating the best achievable performance by a generic MPC controller. This index provides results directly related to process economics, taking into account the required control performance in the face of any given disturbance and process constraints so as to allow comparison between similar process plants. It will be able to provide a measure of how much a plant can be optimized while keeping controlled variables within the bounds of the zone control. 


\section{Some Definitions and Their Use}

Let us now define an index to evaluate just how much room for optimization exists for a given plant when applying a zone constrained model predictive control in the face of disturbances and control bounds. The goal is to define which candidate plant design has the best, most profitable and yet reachable state, enabling the analysis and comparison of slightly different chemical plants in order to find out which one has higher resilience to disturbances, better controllability, lesser product quality giveaway and lower costs. Details of the concept of state reachability can be found in Vidyasagar (2002).

In order to be a worthwhile tool for process design the methodology needs to be carried out independently of other factors such as future choice of MPC algorithm or set of tuning parameters, focusing only on the model response. Minimizing the index for a given plant means finding what out what is the best state that can be reached at the end of the prediction horizon, while subject to zone control. If several chemical plants are being compared with a view to assessing which has better dynamic response, the optimal index is closely related to the best achievable performance any MPC package can achieve.

The success of the control effort made by an MPC controller is its capacity to reject disturbances while optimizing economically the process, and thus the index must account for the eventual economic losses due to the necessary control actions. The methodology presented here will favour solutions which have smooth transitions to the final state and to penalize violations of zone constraints in order to make sure that the dynamic trajectory leading to the optimized steady-state is feasible. Also, restrictions concerning manipulated variables such as their maximum rate of change and maximum and minimum values are incorporated in the analysis.

The index's purpose is to compare different process plants independently of the MPC algorithm and tuning parameters that will be used to control these plants. Considering both the speed of the transient and final values would restrict the validity of the analysis to a certain algorithm and set of tuning parameters, rendering the analysis useless otherwise. Furthermore, introducing criteria such as speed of dynamic response would result in an optimization problem composed of several layers that could be rapidly become intractable for larger systems, which are the main subjects of this work.

The economic MPC optimization index may be used to determine what are the best reachable states for a set of chemical plants subject to disturbances and restrictions 
to inputs and outputs. If these variables, disturbances and restrictions are the same for those plants, the index indicates the better plant from a dynamic behaviour standpoint: the lower the index, the better the state reachability for a given plant. Since commercial MPC packages make use of linear state-space models, which can be identified with relative ease through step tests of the manipulated inputs, here we use a generic linear state-space model to obtain the prediction of process outputs $y$ at the end of the prediction horizon $k+p$ :

$$
\begin{aligned}
& y_{k}=C x_{k} \\
& x_{k+1}=A x_{k}+B \Delta u_{k}+D \Delta d_{k}
\end{aligned}
$$

The model represents a process flowsheet that is assumed to be fixed during the analysis (this approach does not aim to replace early stage process synthesis usually based on steady-state information). The method presents analysis of the most promising flowsheets with regard to zone constrained MPC performance. The goal is to assess which plant is better placed to accommodate disturbances while being optimized by a MPC.

The model defined by equations (1) and (2) makes use of deviation variables. At an arbitrary time instant $k$, it is possible to predict the values of the process outputs using the following procedure:

$$
\begin{aligned}
& y_{k+1}=C x_{k+1}=C A x_{k}+C B \Delta u_{k}+C D \Delta d_{k} \\
& y_{k+2}=C x_{k+2}=C A x_{k+1}+C B \Delta u_{k+1}+C D \Delta d_{k+1} \\
& =C A^{2} x_{k}+\left[\begin{array}{lll}
C A B & C B
\end{array}\right]\left[\begin{array}{c}
\Delta u_{k} \\
\Delta u_{k+1}
\end{array}\right]+[C A D C D]\left[\begin{array}{c}
\Delta d_{k} \\
\Delta d_{k+1}
\end{array}\right] \\
& \vdots \\
& y_{k+2}=C A^{2} x_{k}+[C A B C B]\left[\begin{array}{c}
\Delta u_{k} \\
\Delta u_{k+1}
\end{array}\right]+[C A D C D]\left[\begin{array}{c}
\Delta d_{k} \\
\Delta d_{k+1}
\end{array}\right] \\
& y_{k+p}=C A^{p} x_{k}+\left[C A^{p-1} B C A^{p-2} B \ldots C A^{p-m} B\right]\left[\Delta u_{k} \Delta u_{k+1} \ldots \Delta u_{k+m-1}\right]^{T} \\
& +\left[C A^{p-1} D C A^{p-2} D \ldots C A^{2} D\right]\left[\Delta d_{k} \Delta d_{k+1} \ldots \Delta d_{k+p-3}\right]^{T}
\end{aligned}
$$

where $m$ is the number of time increments of the control horizon, which is also the number of control actions performed. Here we make the assumption that the number of disturbance movements is equal to the prediction horizon. This prediction will later be used to define the economic control resilience index. 


\subsection{Index for Control Bound Violations}

In zone control each controlled variable has a minimum and maximum desired variable but some of these constraints may have more importance than others and, for this reason, when defining the MPC control problem it is common practice to assign each controlled variable a weight value, which establishes the relative priority each bound will have in the solution. For example, constraints relative to process, equipment and environmental safety normally have precedence over those concerning product specifications. Henceforth these weight values will be denominated $W_{i, \text { upper }}$ and $W_{i, \text { lower }}$ meaning respectively the weights for the upper (maximum value) and lower (minimum value) bounds of controlled variable $y_{i}$, where $i=1, \ldots, n y$, and $n y$ is the number of controlled variables. Initially, let us consider the steady-state achieved by the MPC where the plant will operate for much of the time. The questions of smoothness of the transient response shall be dealt with in section 2.4, but for now let us just assume that given enough time the plant will reach steady-state after a series of control and optimization actions. If only the last instant in the prediction is considered, a new problem arises in which the goal is to minimize the sum of the predicted deviations from the control zone for each process output multiplied by its weight. A cost function for this control problem can be defined as follows:

$$
\begin{aligned}
& J_{C V_{k+p}}=\sum_{i=1}^{n y}\left[\left|y_{i, \text { min }}-y_{k+p, i}\right| \cdot W_{i, \text { lower }}+\left|y_{k+p, i-} y_{i, \text { max }}\right| \cdot W_{i, \text { upper }}\right] \\
& \text { if } y_{i, \text { min }} \leq y_{k+p, i} \leq y_{i, \text { max }} \Rightarrow W_{i, \text { upper }}=W_{i, \text { lower }}=0 \\
& \text { if } y_{i, \text { min }}>y_{k+p, i} \Rightarrow W_{i, \text { lower }}>0 \\
& \text { if } y_{k+p, i}>y_{i, \text { max }} \Rightarrow W_{i, \text { upper }}>0
\end{aligned}
$$

where $i=1, \ldots, n y$. In this problem it is of special interest to know if there is a set of manipulated variables, or MVs, that leads the system to a state where all outputs are within their zone constraints at the end of the prediction horizon, and thus $J_{C V_{k+p}}=0$, or alternatively, if there is no ideal solution for equation 4 , what is the final state that minimizes the violation of the control bounds and consequently minimizes $J_{C V_{k+p}}$.

\subsection{An Economic Optimization Index}

MPC controllers found in the chemical industry frequently possess economic optimization functions in addition to the control capabilities. Also recent research has shown that process economics can be optimized directly in the dynamic control problem, which can take advantage of potentially higher profit transients to give superior economic 
performance. Examples of this approach include Amrit et al. (2013) and Strutzel et al. (2013). The optimization is performed by changing the manipulated process inputs when the process finds itself within its control bounds, and degrees of freedom are available to be employed in optimization tasks. In oil refining processes, MVs are often related to process energy cost. For instance, it may be necessary to burn more natural gas in the fired heater in order to increase the temperature of the feed stream to a reactor. If the feed temperature is a MV increasing it has a negative impact on process profitability, which depends on the price of natural gas. Another example would be diesel production in an atmospheric crude oil distillation column. In this process it is often possible to improve the quality of the diesel by reducing its output and, consequently, increasing the atmospheric residue output. However, diesel has a much higher commercial value, so if the flow rate of diesel is a manipulated variable, it is positively correlated to profitability. Other MVs are not strongly correlated to energy costs or product prices and can be altered freely. In inorganic processes the goal is often to maximize chemical reaction conversion and the relation between MV and costs may be less obvious.

It is thus interesting to define for each MV whether it is positively or negatively related to profitability, and to what degree. Let us now define two sets of optimization weights, $V_{j, \min }$ and $V_{j, \max }$, where $j=1, \ldots, n u$, where $n u$ is the number of MVs, which illustrate the optimization direction and relative priority among the various MVs for economic purposes. If a given MV is positively correlated to profitability and at the present moment is not being employed for control actions, it should stay as close as possible to its maximum limit or upper bound. Likewise, if it is negatively related to profitability, it should stay close to its minimum limit or lower bound. In the single layer MPC control scheme, the optimization weights $V_{\min }$ and $V_{\max }$ are very small compared to the control zone weights $W_{i, \text { upper }}$ and $W_{i, \text { lower }}$ in the cost function, and optimization is performed without hindering the control objectives. Ferramosca et al. (2014) provide a formal proof of convergence for such an approach.

Concerning the MVs, it is desired to determine how far they stand from the hard constraints because, depending on the direction of optimization, this distance denotes how much room there is for optimization. A simplified optimization cost function may then be established yielding equation 5, which relates the distance between MVs and their bounds at the prediction's end: 
$J_{M V_{k+p}}=\sum_{j=1}^{n u}\left[\left|u_{k+p, j}-u_{j, \min }\right| \cdot V_{j, \min }+\left|u_{j, \max }-u_{k+p, j}\right| \cdot V_{j, \max }\right]$

Subject to:

$u_{\min } \leq u_{k+p, j} \leq u_{\max }$

where:

$u_{k+p, j}=\sum_{j=1}^{m} \Delta u_{k+j}$

The optimization weights are subject to:

$V_{j, \min } \geq 0, V_{j, \max } \geq 0, V_{j, \min } \cdot V_{j, \max }=0$

where $j=1, \ldots, n u$. The set of restrictions defined by equation 6 was included in order to guarantee that a single economic optimization direction exists for each variable, if there is any: if for a MV of index $j, V_{j, \min }=V_{j, \max }=0$, then the variable doesn't have any optimization direction, being neutral for profitability.

\subsection{Economic MPC Optimization Index}

Adding equations (4) and (5) in a single cost function yields the first form of the economic MPC optimization index:

$$
\begin{aligned}
& J_{k+p}=J_{C V_{k+p}}+J_{M V_{k+p}} \\
& =\sum_{i=1}^{n y}\left[\left|y_{i, \text { min }}-y_{k+p, i}\right| \cdot W_{i, \text { lower }}+\left|y_{k+p, i-} y_{i, \text { max }}\right| \cdot W_{i, \text { upper }}\right] \\
& +\sum_{j=1}^{n u}\left[\left|u_{k+p, j}-u_{j, \text { min }}\right| \cdot V_{j, \text { min }}+\left|u_{j, \max }-u_{k+p, j}\right| \cdot V_{j, \text { max }}\right]
\end{aligned}
$$

Subject to:

$$
\begin{aligned}
& \text { if } y_{i, \text { min }} \leq y_{k+p, i} \leq y_{i, \text { max }} \Rightarrow W_{i, \text { upper }}=W_{i, \text { lower }}=0 \\
& \text { if } y_{i, \text { min }}>y_{k+p, i} \Rightarrow W_{i, \text { lower }}>0 \\
& \text { if } y_{k+p, i}>y_{i, \max } \Rightarrow W_{i, \text { upper }}>0 \\
& V_{j, \text { min }} \geq 0, V_{j, \max } \geq 0, V_{j, \min } \cdot V_{j, \max }=0
\end{aligned}
$$

where $\mathrm{i}=1, \ldots$, ny and $\mathrm{j}=1, \ldots$, nu. The prediction of $y$ at the time instant $k+p$ is given by equation 3 , which can be further simplified by defining the following matrices: 


$$
\begin{aligned}
& \overline{\overline{C_{u}}}=\left[C A^{p-1} B C A^{p-2} B \ldots C A^{p-m} B\right] \quad \overline{\overline{C_{d}}}=\left[C A^{p-1} D C A^{p-2} D \ldots C A^{2} D\right] \\
& \Delta u_{K}=\left[\Delta u_{k} \Delta u_{k+1} \ldots \Delta u_{k+m-1}\right]^{T} \quad \Delta d_{K}=\left[\Delta d_{k} \Delta d_{k+1} \ldots \Delta d_{k+p-3}\right]^{T}
\end{aligned}
$$

Replacing these new terms in equation 3 yields:

$y_{k+p}=C A^{p} x_{k}+\overline{\overline{C_{u}}} \Delta u_{K}+\overline{\overline{C_{d}}} \Delta d_{K}$

The vector $u_{k+p}$ may be calculated as follows:

$u_{k+p}=\sum_{j=1}^{m} \Delta u_{k+j}=I_{m . n u} \cdot \Delta u_{K}$

where:

$$
I_{m . n u}=\underbrace{\left[\begin{array}{cccccccccc}
1 & \cdots & 0 & 1 & \cdots & 0 & & 1 & \cdots & 0 \\
\vdots & \ddots & \vdots & \vdots & \ddots & \vdots & \cdots & \vdots & \ddots & \vdots \\
0 & \cdots & 1 & 0 & \cdots & 1 & & 0 & \cdots & 1
\end{array}\right]}_{m \cdot n u}\} n u
$$

Applying equations 8 and 9 in the cost function 7, and putting the resultant equation into vector form yields a more functional form for the cost function:

$$
\begin{aligned}
& J_{k+p}=\left|y_{\text {min }}-C A^{p} x_{k}-\overline{\overline{C_{u}}} \Delta u_{K}-\overline{\overline{C_{d}}} \Delta d_{K}\right| \cdot W_{\text {lower }} \\
& +\left|C A^{p} x_{k}+\overline{\overline{C_{u}}} \Delta u_{K}+\overline{\overline{C_{d}}} \Delta d_{K}-y_{\text {max }}\right| \cdot W_{\text {upper }} \\
& +\left|I_{\text {m.nu }} \cdot \Delta u_{K}-u_{\text {min }}\right| \cdot V_{\text {min }}+\left|u_{\text {max }}-I_{\text {m.nu }} \cdot \Delta u_{K}\right| \cdot V_{\text {max }}
\end{aligned}
$$

For any system described by a state-space model in the form given by equations 1 and 2, subject to a set of disturbance vectors $\Delta d_{K} \in D_{K}$, the economic MPC optimization index problem is defined by equation 12. The same restrictions as for equation 7 apply.

$$
I_{E_{M P C}}=\min _{\Delta u_{K} \in U}\left(J_{k+p}\right)
$$

\subsection{Ensuring Viable Solutions}

The economic cost function defined in equation 12 guarantees that the final state will be as close as possible to the economic optimal state without violating the MPC constraints but does not consider the transient response. Now we shall modify the cost function to ensure that the transition to the final state is as smooth as possible. In order to achieve this we now introduce two new parameters in the cost function that will 
penalize steep changes in the final predicted values for the states, favouring smooth curves for the controlled variables at the end of the prediction.

These new terms shall be called "soft landing" matrices and will be inversely proportional respectively to the first and to the second order derivatives of the controlled variables at instant $k+p$. By multiplying the economic cost function by the inverses of the soft landing matrices its value will increase proportionally to the slope of the final output prediction. The soft landing matrices will be relevant mostly if $p$, the prediction horizon, is small and the system doesn't have enough time to stabilize. The soft landing matrices for the first and second order derivatives are:

$S L_{1}=I_{n y}-T_{S L 1}\left[\operatorname{diag}\left|y_{k+p}-y_{k+p-1}\right| \cdot \operatorname{diag}\left|y_{\max }-y_{\min }\right|^{-1}\right]$
$S L_{2}=I_{n y}-T_{S L 2}\left[\operatorname{diag}\left|\left[y_{k+p}-y_{k+p-1}\right]-\left[y_{k+p-1}-y_{k+p-2}\right]\right| \operatorname{diag}\left|y_{\max }-y_{\min }\right|^{-1}\right]$

where $T_{S L 1}$ and $T_{S L 2}$ are parameter vectors that define the priority of rejecting sharp moves for each variable. Higher values favour flatter curves at the expense of a more aggressive approach. Values for $T_{S L 1}$ and $T_{S L 2}$ must be assigned so that $S L_{1}$ and $S L_{2}$ are contained in the unit circle. The first order derivative is the difference between the controlled variable's predictions at $k+p$ and $k+p-1$, obtained as follows:

$$
\begin{aligned}
& y_{k+p}-y_{k+p-1}=C A^{p} x_{k}+\left[C A^{p-1} B C A^{p-2} B \ldots C A^{p-m} B\right]\left[\Delta u_{k} \Delta u_{k+1} \ldots \Delta u_{k+m-1}\right]^{T} \\
& +\left[C A^{p-1} D C A^{p-2} D \ldots C A^{2} D\right]\left[\Delta d_{k} \Delta d_{k+1} \ldots \Delta d_{k+p-3}\right]^{T} \\
& -C A^{p-1} x_{k}-\left[C A^{p-2} B C A^{p-3} B \ldots C A^{p-m-1} B\right]\left[\Delta u_{k} \Delta u_{k+1} \ldots \Delta u_{k+m-1}\right]^{T} \\
& -\left[C A^{p-2} D C A^{p-3} D \ldots C A D\right]\left[\Delta d_{k} \Delta d_{k+1} \ldots \Delta d_{k+p-3}\right]^{T}= \\
& {\left[I_{n y}-A^{-1}\right]=\left\{\begin{array}{c}
C A^{p} x_{k}+\left[C A^{p-1} B C A^{p-2} B \ldots C A^{p-m} B\right]\left[\begin{array}{lll}
\Delta u_{k} \Delta u_{k+1} \ldots \Delta u_{k+m-1} & \ldots \\
+ & \left.+C A^{p-1} D C A^{p-2} D \ldots C A^{2} D\right]\left[\Delta d_{k} \Delta d_{k+1} \ldots \Delta d_{k+p-3}\right.
\end{array}\right]^{T}
\end{array}\right\}}
\end{aligned}
$$

Introducing the matrices defined in equation 8 , the derivative given by equation 15 becomes:

$S L_{1}=I_{n y}-$ 


$$
-T_{S L 1}\left[\operatorname{diag}\left|\left[I_{n y}-A^{-1}\right]\left[C A^{p} x_{k}+\overline{\overline{C_{u}}} \Delta u_{K}+\overline{\overline{C_{d}}} \Delta d_{K}\right]\right| \cdot \operatorname{diag}\left|y_{\max }-y_{\min }\right|^{-1}\right]
$$

A similar procedure may be used to obtain the $S L_{2}$, the term related to the second order derivative of the controlled variables at $k+p$, which is the difference between the slope at $k+p$ and the slope at $k+p-1$. The second order derivative may be calculated as follows:

$$
\begin{aligned}
& {\left[y_{k+p}-y_{k+p-1}\right]-\left[y_{k+p-1}-y_{k+p-2}\right]=y_{k+p}-2 y_{k+p-1}+y_{k+p-2}=}
\end{aligned}
$$

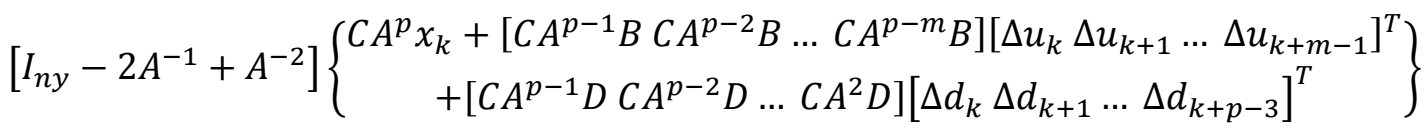

$$
\begin{aligned}
& =\left[I_{n y}-2 A^{-1}+A^{-2}\right]\left[C A^{p} x_{k}+\overline{\overline{C_{u}}} \Delta u_{K}+\overline{\overline{C_{d}}} \Delta d_{K}\right]
\end{aligned}
$$

Then equation 14 becomes:

$$
\begin{aligned}
& S L_{2}=I_{n y}- \\
& -T_{S L 2}\left[\text { diag }\left|\left[I_{n y}-2 A^{-1}+A^{-2}\right]\left[C A^{p} x_{k}+\overline{\overline{C_{u}}} \Delta u_{K}+\overline{\overline{C_{d}}} \Delta d_{K}\right]\right| \text { diag }\left|y_{\max }-y_{\min }\right|^{-1}\right]
\end{aligned}
$$

The soft-landing matrices are incorporated into the cost function, which means that if one or more destabilizing sequences of control actions do exist when under evaluation they would cause the cost function value to explode and thus these sequences would be ignored by the solver. If no smooth solution is available at all the final solution would have a high value that should alert the control engineer.

Besides guaranteeing a smooth transition to steady-state at the prediction's end, we shall now introduce in the cost function a new term that will penalize temporary violations of the control bounds that may occur during the trajectory between the initial state and the optimal final state. This matrix shall be denoted the "error penalization" matrix $(E P)$ and it will increase the cost function value if any of the controlled variables stay out of their control zone:

$$
E P=I_{n y}-
$$

$T_{E P} \sum_{\alpha=k}^{k+p}\left\{\left[\operatorname{diag}\left(p\left|y_{\max }-y_{\min }\right|\right)\right]^{-1} \operatorname{diag}\left(\left|y_{\alpha}-y_{\max }\right| \cdot T_{\text {upper }}+\left|y_{\min }-y_{\alpha}\right| \cdot T_{\text {lower }}\right)\right\}$ where $T_{E P}$ is a parameter vector that defines the priority of rejecting overshooting for each variable, and $\alpha$ is an auxiliary variable that denotes time, whose value varies from $\alpha=k$ (beginning of the prediction) to $\alpha=k+p$ (end of the prediction) in equation 19 . 
So $y_{\alpha}$ is the vector of controlled variables at time alpha, which is being compared to $y_{\max }$ and $y_{\min }$, in order to know if any controlled variable left the control zone during the transient. The sets of parameters $T_{\text {upper }}$ and $T_{\text {lower }}$ must obey the following restrictions:

$$
\begin{aligned}
& \text { if } y_{i, \text { min }} \leq y_{k+p, i} \leq y_{i, \text { max }} \Rightarrow T_{i, \text { upper }}=T_{i, \text { lower }}=0 \\
& \text { if } y_{i, \text { min }}>y_{k+p, i} \Rightarrow T_{i, \text { lower }}>0 \\
& \text { if } y_{k+p, i}>y_{i, \text { max }} \Rightarrow T_{i, \text { upper }}>0
\end{aligned}
$$

where $i=1, \ldots, n y$. These restrictions guarantee that $E P$ will decrease if, during the transient, any controlled variable overshoots. If that happens, the solution will be penalized even if the final state is within its control zone. Matrices $T_{\text {lower }}$ and $T_{\text {upper }}$ indicate how strongly this overshooting will be rejected.

Finally, we can reach the final form for the economic MPC optimization index by multiplying the cost function given by equation 12 by the inverse of the determinants of the soft landing and the error penalization matrices, $S L_{1}, S L_{2}$ and $E P$ :

$$
I_{E_{M P C}}=\min _{\Delta u_{K} \in U}\left(|E P|^{-1}\left|S L_{1}\right|^{-1}\left|S L_{2}\right|^{-1} J_{k+p}\right)
$$

The new cost function will cause the optimization algorithm to discard overshooting solutions that could be otherwise selected if equation 12 was used. Evidently, if $p$ is sufficiently large all control actions and their effects will have taken place at the end of the prediction, $k+p$, and thus, $S L_{1}$ and $S L_{2}$ will be identity matrices. If however, $p$ is small or if the set of control actions results in an unstable response, the index cost function will increase and the solution will be penalized.

The vectors $T_{S L 1}, T_{S L 2}, T_{E P}, T_{\text {upper }}$ and $T_{\text {lower }}$ all require manual tuning. Their values should reflect prioritization among controlled variables as well the desired balance between steady-state optimization and penalization for eventual issues in the transient behaviour. The larger the values, the greater the penalties for lack of a smooth transition to the final state and for violations of the control zones. The exact values that should be assigned depend on the number of variables (a larger number of variables increases their cumulative effect) and the desired penalty. For example, one could tune $T_{E P}, T_{\text {upper }}$ and $T_{\text {lower }}$ in such a way that $|E P|^{-1}=1+1.5 / n y$, if a single variable stays unbounded for $50 \%$ of the transient, and $|E P|^{-1}=1+1.75 \cdot 1.5 / n y$, if additionally another variable is unbounded for $75 \%$ of the transient, and so on. Another possibility is setting $T_{S L 1}$ and 
$T_{S L 2}$ in such a way as to provide soft-landing matrices that follow, approximately, $\left|S L_{1}\right|^{-1}=\prod \sqrt[n y]{1+y_{k+p}^{\prime} / y_{k+p}}$ and $\left|S L_{2}\right|^{-1}=\prod \sqrt[n y]{1+y_{k+p}^{\prime \prime} / y_{k+p}}$. The tuning choices ultimately depend on the control engineer's judgement about the correct balance between steady-state and transient performances, both of which are important elements of the analysis. The concept of "optimal tuning" does not apply here: what is necessary is that the selection of tuning parameters reflects adequately the criteria by which process performance is going to be judged. The parameters must be the same for all flowsheets resulting in the use of the same criterion.

\subsection{Exploring the Relation between the Regulatory Control Layer, MPC Layer and the Economic MPC Optimization Index}

The approach presented in this paper is based on the premise that disturbances are known and estimated a priori and follow a given time-dependant profile. While this may seem restrictive, this choice reflects the nature of the dual layer control strategy used for plant control and also that in many chemical processes often the MPC layer disturbances are known and planned ahead of time by operating staff. Examples of MPC layer disturbances include events such as changes in the feed composition and product specifications, programmed equipment shutdowns, tank switches, and changes in pipeline alignment. Here we compare for each plant how planned one-off occurrences, which are very frequent and impact process profitability. Disturbances that may be modelled stochastically, such as observational noise, are normally dealt with by the regulatory control layer, which is much faster. The control engineer must keep in mind that the regulatory layer is part of the plant which is being evaluated and modifying it changes the model and analysis results.

In the case study provided in section 3 the linear models that define each plant are closed-loop models involving multi-loop feedback controllers. The sample time used for the state-space models was 10 minutes while PID controller sample time was set at 1 second at the simulation. The large difference in speed between MPC and PID variables greatly diminishes the index sensitivity to the regulatory control structure: the PIDs could easily and rapidly bring its CVs back to their set points, should they be disturbed. Also, for all plants those controllers proved themselves easy to tune and had excellent performance. So in this case, the effect of the regulatory control layer was small enough to be ignored. In other processes however, these effects may be larger, and the index may 
vary considerably according to the selection of control schemes and tuning parameters. For example, if the regulatory control is too slow or difficult to tune, or if its actuators run at their saturation limits, then the regulatory layer will have greater impact on the models and, consequently, on the index.

In the following sections the method is applied to candidate flowsheets for an oil distillation process unit. For this approach the candidate flowsheets must have their stability confirmed before applying the economic MPC optimization index. The classical approach is to use the well-known stability theorem which states that a linearly time invariant (LTI) system is stable if all eigenvalues of model matrix A have magnitude less than one, i.e. lie inside the unit circle. According to this definition all flowsheets discussed in sections 3 and 4 are open loop stable. Details concerning MPC and state-space stability can be found in Mayne et al. (2000) and Oliveira et al. (1999).

\section{Case of Study - Economic Disturbance Index for an Oil Distillation Process Unit}

In order to demonstrate how the economic disturbance cost index may be applied to provide solutions to industrial scale problems three possible designs for a crude oil distillation plant shall be presented. Also a common set of controlled and manipulated variables for the MPC control problem will be defined. As the index is related to the best possible solution for this control problem, its value will indicate which plant can be better controlled by a well-tuned MPC controller. In section 7, the index will be evaluated for each of four different scenarios.

\subsection{Describing the Control Problem}

The models of the plants presented here were obtained through dynamic simulation using Honeywell's UniSim ${ }^{\circledR}$ software. They have a very similar design, but present key differences. These differences represent significant design decisions that the project engineers have to make through the process of specifying the layout and dimensions of a chemical plant. The distillation plants are rather simple and have a typical configuration. The problem has 36 components, 8 local PI controllers, 21 subsystems and the column has 29 trays. This is considerable larger than the examples reported above. The base case, or plant 1 , can be seen below in figure 2 . 
The process simulated has a realistically drafted layout for a medium sized crude oil distillation process unit. The distillation column generates 5 different product streams (Naphtha, Kerosene, Light Diesel, Heavy Diesel and Residue). The Kerosene, Light Diesel, Heavy Diesel and Residue product streams are used to preheat the crude oil feed from $25^{\circ} \mathrm{C}$ to about $220^{\circ} \mathrm{C}$ in two series of heat exchangers, yielding high energetic efficiency. After the first series the oil reaches an adequate temperature to enter the desalter drum where salt is removed from the oil. After passing through the second series of exchangers, the pre-heated oil enters a fired heater where its temperature is increased to $320-380{ }^{\circ} \mathrm{C}$. The hot crude is then fed to the distillation column where the product streams are obtained. The cold light diesel and cold heavy diesel streams are mixed together to generate the "Pool Diesel" stream, whose properties will be used to evaluate the cost index.

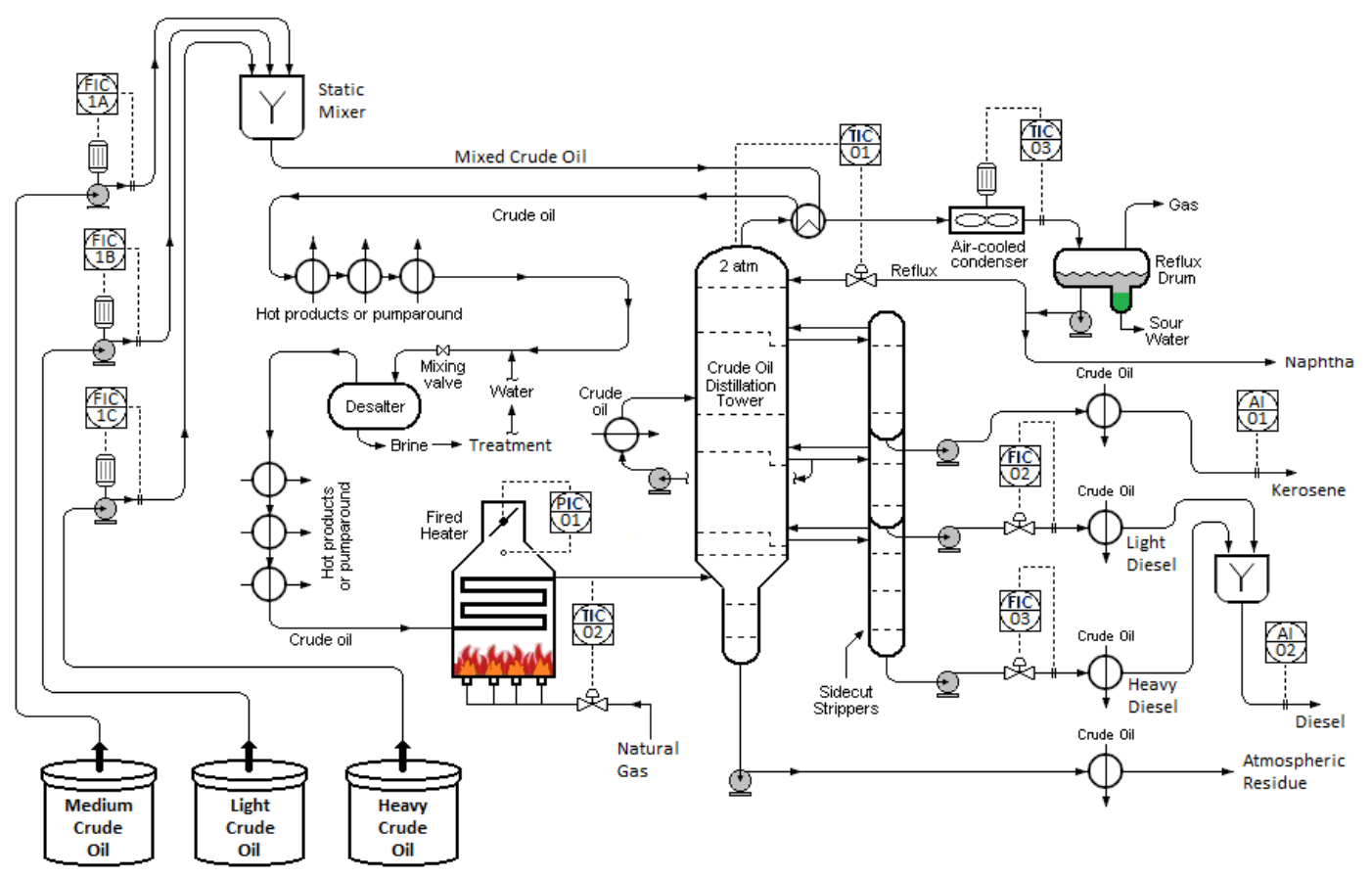

Figure 2 - Plant 1 - Simplified Process Flow Sheet

There are three different types of crude oil available for processing in the three distillations units: medium $\left(29.0^{\circ} \mathrm{API}\right)$, light $\left(32.3^{\circ} \mathrm{API}\right)$ and heavy $\left(26.2^{\circ} \mathrm{API}\right)$ crude oils. The light oil provides better yields of the more valuable naphtha, diesel and kerosene and lower yield of the less desired atmospheric residue. However, it is the most expensive. The heavy oil provides poor yields of the lighter, more valuable products but on the other hand it is considerably cheaper. Table 1 provides example costs for the crude oils and the prices for the products, which will be used in the simulation to calculate the profitability 
of the process. Those values are based on the average prices for the Brazilian market in 2015, which can be found on ANP (National Agency of Petroleum and Natural Gas) website.

\begin{tabular}{|c|c|c|}
\hline Medium Crude Oil & $\mathrm{US} \$ / \mathrm{m}^{3}$ & 452.69 \\
\hline Heavy Crude Oil & $\mathrm{US} \$ / \mathrm{m}^{3}$ & 419.17 \\
\hline Light Crude Oil & $\mathrm{US} \$ / \mathrm{m}^{3}$ & 496.76 \\
\hline Naphtha & $\mathrm{US} \$ / \mathrm{m}^{3}$ & 484.84 \\
\hline Kerosene & $\mathrm{US} \$ / \mathrm{m}^{3}$ & 557.83 \\
\hline Diesel & $\mathrm{US} \$ / \mathrm{m}^{3}$ & 551.21 \\
\hline Residue & $\mathrm{US} \$ / \mathrm{m}^{3}$ & 470.17 \\
\hline
\end{tabular}

Table 1 - Crude Oil Costs and Product Prices

The optimization problem for Plant 1, which will be exactly the same for Plants 2, 3 and 4, consists of maximizing the share of Heavy Crude Oil in the feed while minimizing the share of Light Crude Oil, bringing costs down, and at the same time increasing the yield of higher priced Diesel and Kerosene in the products. The product specifications, which act as restrictions for profit maximization, are shown in table 2:

\begin{tabular}{ccccc}
\hline & Controlled Variables Description & Unit & Maximum & Minimum \\
\hline $\boldsymbol{y}_{\mathbf{1}}$ & Cetane Index DIESEL & & - & 42 \\
$\boldsymbol{y}_{\mathbf{2}}$ & Flash Point DIESEL & $\mathrm{C}$ & - & 55 \\
$\boldsymbol{y}_{\mathbf{3}}$ & ASTM D86 DIESEL 65\% & $\mathrm{C}$ & - & 250 \\
$\boldsymbol{y}_{\mathbf{4}}$ & ASTM D86 DIESEL 85\% & $\mathrm{C}$ & 350 & - \\
$\boldsymbol{y}_{\mathbf{5}}$ & ASTM D86 DIESEL 95\% & $\mathrm{C}$ & 370 & - \\
$\boldsymbol{y}_{\mathbf{6}}$ & Freezing Point DIESEL & $\mathrm{C}$ & -15 & - \\
$\boldsymbol{y}_{\mathbf{7}}$ & Density (15 C) DIESEL & $\mathrm{kg} / \mathrm{m}^{3}$ & 860 & 820 \\
$\boldsymbol{y}_{\mathbf{8}}$ & ASTM D86 KEROSENE 100\% & $\mathrm{C}$ & 300 & - \\
$\boldsymbol{y}_{\mathbf{9}}$ & Flash Point KEROSENE & $\mathrm{C}$ & - & 38 \\
$\boldsymbol{y}_{\mathbf{1 0}}$ & Density (15 C) KEROSENE & $\mathrm{kg} / \mathrm{m}^{3}$ & 840 & 775 \\
$\boldsymbol{y}_{\mathbf{1 1}}$ & Freezing Point KEROSENE & $\mathrm{C}$ & -47 & - \\
\hline
\end{tabular}

Table 2 - Description and limits for the controlled variables.

It is also interesting to control Kerosene's properties. Table 2 below provides a list of the controlled variables whose limits must be enforced by an MPC controller and must be taken into account while evaluating the economic disturbance cost index. The values below are true specifications for the fuels marketed in the European Union.

The manipulated variables available to the MPC controller and their limits can be found in table 3. The plant has a number of PID feedback controllers and the plant statespace model is a closed loop model. In a classic two layer control framework, the MPC manipulated variables are the PID controllers' set points. 


\begin{tabular}{ccccc}
\hline & Manipulated Variables Description & Unit & Maximum & Minimum \\
\hline $\boldsymbol{u}_{\mathbf{1}}$ & Temperature 01 tray TIC01.SP & $\mathrm{C}$ & 70 & 40 \\
$\boldsymbol{u}_{\mathbf{2}}$ & Temperature Fired Heater TIC02(B).SP & $\mathrm{C}$ & 380 & 320 \\
$\boldsymbol{u}_{\mathbf{3}}$ & Light Diesel Output FC02.SP & $\mathrm{m}^{3} / \mathrm{h}$ & $270(*)$ & 0 \\
$\boldsymbol{u}_{\mathbf{4}}$ & Heavy Diesel Output FC03.SP & $\mathrm{m}^{3} / \mathrm{h}$ & $65(*)$ & 0 \\
$\boldsymbol{u}_{\mathbf{5}}$ & Medium Crude Flow Rate FC01A.SP & $\mathrm{m}^{3} / \mathrm{h}$ & $800(* *)$ & 0 \\
$\boldsymbol{u}_{\mathbf{6}}$ & Light Crude Flow Rate FC01B.SP & $\mathrm{m}^{3} / \mathrm{h}$ & $800(* *)$ & 0 \\
$\boldsymbol{u}_{\mathbf{7}}$ & Heavy Crude Flow Rate FC01C.SP & $\mathrm{m}^{3} / \mathrm{h}$ & $800(* *)$ & 0 \\
\hline
\end{tabular}

Table 3 - Description and limits for the manipulated variables. *Total diesel production (sum of $u_{3}$ and $u_{4}$ ) must be at least $85 \mathrm{~m}^{3} / \mathrm{h}$. **The sum of $u_{5}, u_{6}$ and $u_{7}$ must be equal to $800 \mathrm{~m}^{3} / \mathrm{h}$, keeping the total feed flow constant.

A slop recycle will be used as a measured disturbance. Processes such as atmospheric or vacuum distillation produce several main cuts as well as slop cuts. Slop oil is the collective term for mixtures of heavy fractions of oil, chemicals and water derived from a wide variety of sources in refineries or oil fields, often forming emulsions. For example, in a vacuum distillation unit the slop oil and water are separated by gravity in the vacuum drum. It is also formed when tank wagons and oil tanks are cleaned and during maintenance work or in unforeseen oil accidents. Slop oil formation can be reduced but cannot be avoided and the need to dispose of it results in one of the largest challenges in the everyday operation of an oil refinery.

The slop cuts produced during the operation of oil refining are conventionally stored in large oil lagoons or tanks to receive chemical treatment so as to enable them to be recycled to process units such as fluid catalytic cracking or, very often, atmospheric distillation units. Therefore, slop oil must be incorporated into the process feed from time to time. In the distillation unit simulated in this work, it is possible to treat the recycle of slop oil as a disturbance and measure the impact of changes in its flow rate in the controlled variables. The same set of variables was defined for all three plants, and a state space model for every pair of input and output has been identified through step tests carried out by dynamic simulation. These models are shown in the appendix.

Plant 2 is essentially the same process as plant 1 , with the exception of the presence of two product tanks which collect respectively the kerosene and pool diesel output streams. The kerosene tank is $616 \mathrm{~m}^{3}$ and the diesel tank is $1692 \mathrm{~m}^{3}$, which implies a residence time of 10 hours for both the kerosene and diesel streams if flow rates remain at their steady state values. In plant 2, instead of being concerned about the properties of distillation column side streams of diesel and kerosene as in plant 1 , it is desired to control 
the properties of the diesel and kerosene streams exiting the product tanks. Plant 2 is presented in figure 4 . The virtual analysers AI01 and AI02 are placed in different positions compared to figure 3 , i.e., after the diesel and kerosene product tanks instead of after the column.

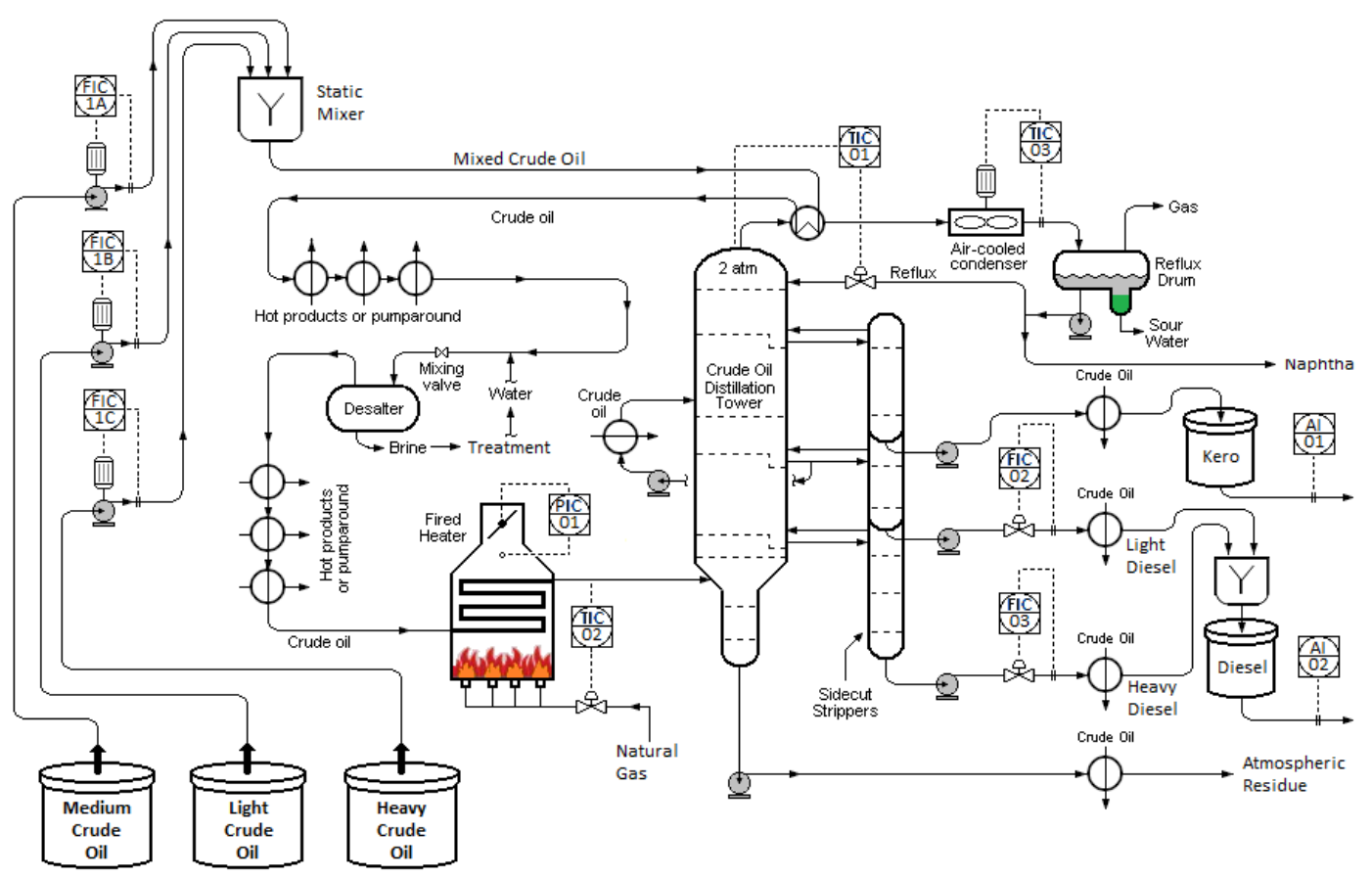

Figure 3 - Plant 2 - Simplified Process Flow Sheet - Plant with Product Tanks.

Plant 3 and 4 are also very similar to plant 1 , but with distillations columns of remarkably different dimensions. Plant 3's column is of increased size compared to plants 1 and 2's, while Plant 4's has a smaller column accompanied of a pre-flash drum that removes the lighter fractions such as $\mathrm{C} 1-\mathrm{C} 4$ gases and light naphtha and an extra fired heater. This new fired heater ensures the feed has an appropriate relation between gas and liquid phases. Plant 3's column may be considered to be slightly oversized for the nominal feed flow rate of $800 \mathrm{~m}^{3} / \mathrm{h}$, and the interesting point here is the slower dynamic response provided by a larger column. Plant 4's pre-flash drum has a volume of $12.56 \mathrm{~m}^{3}$. In section 5 it shall be discussed how these differences may affect the process controllability by a MPC controller. The differences in the sizing parameters of the columns in plant 1,2,3 and 4 (column height and number of trays are the same) are shown in table 4:

\begin{tabular}{cccc}
\hline & Plant 1 and 2 & Plant 3 & Plant 4 \\
\hline Column Diameter $(\mathbf{m})$ & 13.7 & 15 & 11.62 \\
Tray Space $(\mathbf{m})$ & 0.60 & 0.70 & 0.51 \\
Tray Volume $\left(\mathbf{m}^{\mathbf{3}}\right)$ & 88.45 & 123.7 & 52.1 \\
\hline
\end{tabular}




\begin{tabular}{cccc}
\hline Weir Height $(\mathbf{m m})$ & 50 & 65 & 42.40 \\
Weir Length $(\mathbf{m})$ & 10.0 & 14.0 & 7.9 \\
Downcomer Volume $\left.\mathbf{( m}^{\mathbf{3}}\right)$ & 0.08836 & 0.1 & 0.08836 \\
Internal Type & Sieve & Bubble Cap & Sieve \\
\hline
\end{tabular}

Table 4 - Column Sizing Parameters for Plants 1, 2, 3 and 4.

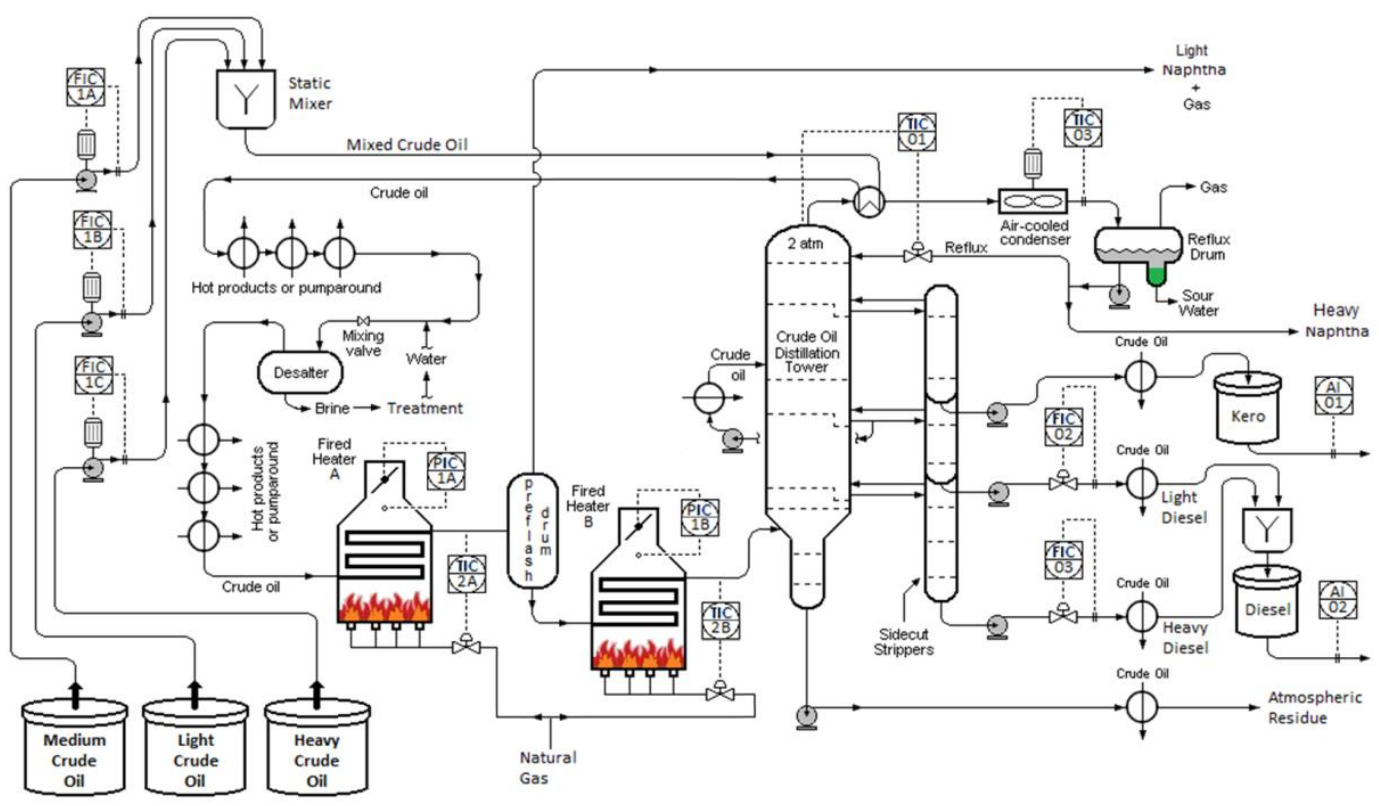

Figure 4 - Plant 4 - Simplified Process Flow Sheet - Plant with Pre-flash Drum.

\subsection{State-Space Model Formulation}

The state-space formulation for this plant is presented in Strutzel et al. (2013) and is built upon the transfer functions that can be obtained from the analytical form of the step response of the system, a convenient and common way of obtaining the models of a chemical plant. Therefore, this formulation was selected for use in the case study discussed in this section. More details and further developments about this kind of model representation, designated output prediction oriented model (OPOM), which was first proposed by Rodrigues and Odloak (2000), can be found in Martins et al. (2013) and González et al. (2007).

\subsection{Measuring the Economic Impact of the Control Effort}

In the case of an oil distillation unit, it is necessary to burn more natural gas in the fired heater in order to increase the temperature of the feed stream to the column. Therefore, since the feed temperature is a manipulated variable increasing it has a negative impact on process profitability, which depends on the price of natural gas. Thus, $u_{2}$ is negatively correlated to profitability. 
The MPC controller also should be able to maintain the diesel output at the maximum value that guarantees a specified product, without reducing output unnecessarily. The commercial value of the residue stream is much lower than that of the diesel, thus transferring hydrocarbons from the diesel to residue decreases revenue. Therefore, $u_{4}$ is positively correlated to profitability.

Finally, the composition of the feed is also defined by the MPC controller. It is able to manipulate the flow rate of Light, Medium and Heavy oil crudes in order to keep specifications within constraints, decreasing the volume of heavy oil and increasing light oil when necessary. Once more, there is a trade-off between profitability and product specifications, because lighter crudes usually generate better products but also cost more. The medium crude will make the bulk of the feed and has properties in between those of heavy and light oil.

\subsection{Defining Parameters for measuring Economic Control Cost}

As discussed in section 2, each of the process inputs and outputs requires a weighting parameter to which the analysis is highly sensitive. In this section adequate values for these shall be defined using market prices for the crude oil feed, product streams, energy costs and data from the simulation.

\section{$u_{1}-$ Temperature 01 tray (TIC01.SP)}

Concerning the distillation column top temperature control, there is an energetic cost to decreasing it due to the fact that more cooling water will be spent in order to increase the reflux flow rate. Calculating the cost of generating cooling water is a complex task, but it can all nevertheless be assumed that this cost is insignificant compared the other costs involved and therefore will be considered equal to zero. Thus, it is assumed that $V_{1, \min }=V_{1, \max }=0$.

\section{$u_{2}-$ Temperature Fired Heater (TIC02.SP / TIC02B.SP)}

It is possible to establish a relation between the crude oil temperature at the fired heater outlet and the heat duty for plant 1,2 and 3 . At a fixed feed flow of $800 \mathrm{~m}^{3} / \mathrm{h}$, the simulation provides the values found in table 5 below:

\begin{tabular}{cc}
\hline $\mathbf{T}(\mathbf{C})$ & $\mathbf{Q}($ Mcal/h) \\
\hline $\mathbf{3 3 0 . 0 7}$ & 61,066 \\
$\mathbf{3 3 4 . 1 1}$ & 62,406 \\
$\mathbf{3 3 5 . 1 3}$ & 62,676 \\
$\mathbf{3 3 6 . 1 0}$ & 62,966 \\
\hline
\end{tabular}




\section{$\mathbf{3 4 0 . 0 0} 64,332$ \\ Table 5 - Energy consumption by the fired heater}

where $\mathrm{T}$ is the fired heater outlet temperature and $\mathrm{Q}$ is the heat duty. From the values above we obtain the increase in energy consumption relative to the increase in outlet temperature, as shown in table 6:

\begin{tabular}{ccc}
\hline$\Delta \mathbf{T}$ & $\begin{array}{c}\Delta \mathbf{Q} \\
(\mathbf{C})\end{array}$ & $\begin{array}{c}\Delta \mathbf{Q} / \Delta \mathbf{T} \\
(\mathbf{M c a l} / \mathbf{h})\end{array}$ \\
\hline $\mathbf{4 . 0 4}$ & $1,339.8$ & 331.4 \\
$\mathbf{1 . 0 2}$ & 270.8 & 265.5 \\
$\mathbf{0 . 9 7}$ & 289.8 & 299.2 \\
$\mathbf{3 . 9 0}$ & $1,365.5$ & 349.9 \\
\hline
\end{tabular}

Table 6 - Increase in energy consumption by the fired heater

Clearly, the energy cost is dependent on the starting temperature and the relationship is nonlinear. It also depends on the feed flow to the column. However, for control purposes the values are close enough and an average value can be used without any compromises to control performance. The average of $\Delta \mathrm{Q} / \Delta \mathrm{T}=311.5 \mathrm{Mcal} /(\mathrm{C} . \mathrm{h})$ shall be considered at any feed flow rate. Considering the Lower Heating Value (LHV) of natural gas equal to $8.747 \mathrm{Mcal} / \mathrm{m}^{3}$ and a natural gas price of $0.33 \mathrm{US} \$ / \mathrm{m}^{3}$, the cost related to $u_{2}$ can be calculated as follows:

$$
\begin{aligned}
& V_{2, \text { min }}=\frac{\Delta Q / \Delta T}{L H V} P_{N G}=\frac{311.5 \mathrm{Mcal} / \mathrm{C} . h}{8.747 \mathrm{Mcal} / \mathrm{m}^{3}} 0.33 \frac{U S \$}{\mathrm{~m}^{3}}=11.75 \frac{U S \$}{\mathrm{C} . h} \\
& V_{2, \text { max }}=0
\end{aligned}
$$

For plant $4 u_{2}$ is defined as the temperature at the fired heater B outlet. Because the light components are separated from the feed at the pre-flash drum, the feed properties and, thus the heat exchange coefficients, are slightly different. However, for the sake of simplicity this difference will be ignored since its effects are very small.

\section{$u_{3} / u_{4}-$ Light Diesel Output (FC02.SP) and Heavy Diesel Output (FC03.SP)}

In this example the light and heavy diesel streams are combined to produce the "pool diesel". The kerosene output and diesel output are placed sequentially in the boiling point curve and therefore transferring hydrocarbons between these streams is part of normal of everyday operations. However, the UNISIM simulation used in this example considers a fixed kerosene output in order for the simulator to be able to solve the fluid flow dynamic equations. However there is a variable flux between the diesel output and 
the residue output and thus the optimization gain is defined as the price difference between these streams:

$$
\begin{aligned}
& V_{3, \text { max }}=V_{4, \text { max }}=P_{\text {diesel }}-P_{\text {residue }}=551.21-470.17=81.04 \frac{U S \$}{m^{3}} \\
& V_{3, \text { min }}=V_{4, \text { min }}=0
\end{aligned}
$$

As discussed at the beginning of this section, increasing heavy diesel output reduces the residue output and thus increases profitability. In this case study the separation between light and heavy diesel output has no commercial importance but in most refineries these hydrocarbon streams have different destinations, such as being fed to different hydrotreating or hydrocracking process units, and therefore they may have different commercial values. These possibilities are not considered here.

\section{$u_{5}-$ Medium Crude Oil Feed Flow Rate (FC01A.SP)}

Given a set of flow rate values for each of the crude oils that compose the feed to the distillation column, at any given time the average price of the oil processed is given by:

$$
P_{\text {ave }}=\frac{452.69 u_{5}+496.76 u_{6}+419.17 u_{7}}{u_{5}+u_{6}+u_{7}} \frac{U S \$}{m^{3}}
$$

The difference between average oil price and the medium crude will provide the optimization coefficient.

$$
\Delta P_{\text {medium }}=P_{\text {medium }}-P_{\text {ave }}
$$

Therefore the following rule may be defined to obtain $V_{5, \min }$ and $V_{5, \max }$ :

$$
\begin{aligned}
& \text { if } \Delta P_{\text {medium }}>0 \Rightarrow V_{5, \text { max }}=0, \quad V_{5, \text { min }}=\Delta P_{\text {medium }} \\
& \text { if } \Delta P_{\text {medium }}<0 \Rightarrow V_{5, \text { max }}=-\Delta P_{\text {medium }}, V_{5, \text { min }}=0 \\
& \text { if } \Delta P_{\text {medium }}=0 \Rightarrow V_{5, \text { max }}=V_{5, \text { min }}=0
\end{aligned}
$$

In the third case, all feed is already entirely composed of medium crude and average oil price doesn't change with changes in $u_{5}$.

\section{$u_{6}-$ Light Crude Oil Feed Flow Rate (FC01B.SP)}

In a similar manner to the approach used for $u_{5}$, the difference between average oil price and the medium crude will provide the optimization coefficient $V_{6, \min }$, and since the light oil is the most expensive, the maximization coefficient will always be zero. 


$$
V_{6, \text { min }}=\Delta P_{\text {light }}=P_{\text {light }}-P_{\text {ave }} \quad V_{6, \text { max }}=0
$$

\section{$u_{7}-$ Heavy Crude Oil Feed Flow Rate (FC01C.SP)}

In a similar manner to the approach used for $u_{5}$ and $u_{6}$, the difference between an average oil price and the medium crude will provide the optimization coefficient $V_{7, \max }$, and since the light oil is the most expensive, the minimization coefficient will always be zero:

$$
V_{7, \text { max }}=\Delta P_{\text {heavy }}=P_{\text {ave }}-P_{\text {heavy }} \quad V_{7, \text { min }}=0
$$

\section{$d$ - Disturbance slop recycle}

While in operating refineries slop streams require an expensive previous treatment in order to be incorporated into the feed and reprocessed, in this study this element of process economics will be ignored. It will not change the analysis since the MPC is not able to determine slop flow rate in first place. Being a disturbance, the slop will not have a price tag in the optimization problem, and thus may be considered a feed component whose hydrocarbons are available for "free" for they do not need to be acquired and, once these slop hydrocarbons replace crude oil in the feed and are recovered and incorporated in the product cuts, they cause the overall index to decrease. At the same time, since slop is composed mostly of very heavy, difficult to process oil cuts it diminishes the maximum quantity of cheap heavy oil that can be processed and increases the energy consumption, and in its turn these effects increase the index. The absolute values are not consequential: the goal is to evaluate the relative performance of each plant which is going to behave differently due to the presence of slop in the feed.

\section{Controlled Variables}

To avoid having controlled variables out of their control zones due to the optimization efforts, the parameters $W_{\text {upper }}$ and $W_{\text {lower }}$ must have high enough values that the cost generated by one or more process outputs outside their control zone is much higher than the cost due to optimization. From an economic perspective, since product specifications are requirements for the product to be saleable, the optimization weight for each controlled value can be defined as the value of the relevant product stream as provided in Table 1 . Since $y_{1}$ to $y_{7}$ are related to diesel specifications, the cost of violating their restrictions will be equal to the diesel price multiplied by diesel output, which is defined as the sum of $u_{3}$ and $u_{4}$. In this case study the possibility of selling diesel and 
kerosene as fuel oil is not being considered, and such a procedure is also very unlikely in industrial operations.

$$
551.21 \frac{U S \$}{m^{3}}\left(u_{3}+u_{4}\right) \frac{m^{3}}{(10 \mathrm{~min})}=551.21\left(u_{3}+u_{4}\right) \frac{U S \$}{(10 \mathrm{~min})}
$$

Similarly, $y_{8}$ to $y_{11}$ are related to kerosene specifications, and their weight in the optimization problem will be equal to the kerosene price multiplied by kerosene output, which is kept fixed at $61.29 \mathrm{~m}^{3} / \mathrm{h}$, or $10.215 \mathrm{~m}^{3} /(10 \mathrm{~min})$.

$$
557.83 \frac{U S \$}{m^{3}} 10.215 \frac{m^{3}}{(10 \mathrm{~min})}=5698.23 \frac{U S \$}{(10 \mathrm{~min})}
$$

Hence, the set of rules below was adopted for defining the weights of each controlled variable in the cost function:

$$
\begin{aligned}
& \text { if } y_{i, \min } \leq y_{k+p, i} \leq y_{i, \max } \Rightarrow W_{i, \text { lower }}=W_{i, \text { upper }}=0 \\
& \text { if } y_{i, \min }>y_{k+p, i} \Rightarrow W_{i, \text { lower }}=551.21\left(u_{3}+u_{4}\right), \quad i=1,2,3,4,5,6,7 \\
& \text { if } y_{k+p, i}>y_{i, \max } \Rightarrow W_{i, \text { upper }}=551.21\left(u_{3}+u_{4}\right), \quad i=1,2,3,4,5,6,7 \\
& \text { if } y_{i, \min }>y_{k+p, i} \Rightarrow W_{i, \text { lower }}=5698.23, \quad i=8,9,10,11 \\
& \text { if } y_{k+p, i}>y_{i, \max } \Rightarrow W_{i, \text { upper }}=5698.23, \quad i=8,9,10,11
\end{aligned}
$$

\section{Results and Discussion}

In this section the results obtained through the application of the economic MPC optimization index to assessing alternative distillation plant designs are presented. The optimization problem was solved using the interior-point routine available in the optimization toolbox in Matlab. It is also worth noting that the problem has nonlinear constraints and required a solver able to deal with disjunctive programming. However, in some cases the results proved to be sensitive to the initial point chosen, and the optimization algorithm sometimes reached a local minimum instead of a global one. To avoid this shortcoming an iterative "genetic" algorithm strategy was implemented consisting of using the previous solution the new starting point, but randomly modifying it so as to implement "mutations", then evaluating the cost function and storing the new solution if it was better than the previous one. The random mutations were constrained to be within $\pm 25 \%$ of the acceptable range for each manipulated variable and 100 iterations were permitted using this methodology in each case. While we believe the solutions presented are global, no formal proof will be provided. 


\subsection{Scenario 1 - Simultaneous Control and Optimization without Disturbance}

The four different plants were given the same starting point, i.e. the same initial values for the controlled and manipulated variables. Given this set of values, it is necessary to obtain the initial state vector, $x_{k}$ for each plant. For the state-space model employed, a completely null state vector $\left(x_{k}=0\right)$ is the model's origin, or the equivalent of having the plant at the exact same steady state used during the step test model identification. To test a different starting points equation 21 gives initial states:

$$
y_{k}=C x_{k} \Rightarrow x_{k}=C^{-1} y_{k}
$$

but there are an infinite number of potential solutions to equation 21 and future states may also be affected the choice of $x_{k}$, as can be seen by equation 3 . To make sure a "coeteris paribus" comparison is possible, it is convenient to implement equation 21 as a restriction that ensures the selection of a stable state, which satisfies equation 21 while having no impact on future states $x_{k+1}, x_{k+2}, \ldots, x_{k+p}$. The restriction defined by equation 22 satisfies these criteria:

$$
0=\sum_{\mathrm{z}=0}^{\mathrm{p}}\left|\mathrm{y}_{\mathrm{k}}-\mathrm{CA}^{\mathrm{z}} \mathrm{x}_{\mathrm{k}}\right|
$$

For all the plants studied it was possible to obtain $x_{k}$ that satisfied equations 21 and 22. The same values for the controlled and manipulated variables resulted in distinct vectors $x_{k}$ since each plant has its own model. Although we recommend several distinct starting points to be tested for thorough analysis, due to lack of space our analysis will be carried out using the single one given in table 7 :

\begin{tabular}{cccccccccccc}
\hline & $\mathbf{y}_{\mathbf{1}}$ & $\boldsymbol{y}_{\mathbf{2}}$ & $\boldsymbol{y}_{\mathbf{3}}$ & $\boldsymbol{y}_{\mathbf{4}}$ & $\mathbf{y}_{\mathbf{5}}$ & $\mathbf{y}_{\mathbf{6}}$ & $\boldsymbol{y}_{\mathbf{7}}$ & $\boldsymbol{y}_{\mathbf{8}}$ & $\boldsymbol{y}_{\mathbf{9}}$ & $\mathbf{y}_{\mathbf{1 0}}$ & $\mathbf{y}_{\mathbf{1 1}}$ \\
\hline $\begin{array}{c}\text { System } \\
\text { origin }\end{array}$ & 44.00 & 81.76 & 288.82 & 319.42 & 341.42 & -25.47 & 826.79 & 251.11 & 49.79 & 812.88 & -68.38 \\
$\begin{array}{c}\text { Starting } \\
\text { point }\end{array}$ & 43.28 & 83.95 & 292.39 & 318.69 & 341.70 & -25.60 & 829.33 & 251.48 & 50.82 & 815.75 & -68.62 \\
\hline
\end{tabular}

Table 7 - New initial values for process outputs.

The assigned value for controlled variable $y_{1}$, the Cetane Index, is set lower than its required value of 46 . This requires control actions to bring $y_{1}$ back to its control zone and this control effort may limit the room for optimization. In this first scenario, which may serve as a basis of comparison with other cases, the processes are at steady-state and there is no disturbance. Optimization was carried out with the restriction of constant total feed flow and the set of parameters introduced in section 4 for plants 1,2, 3 and 4, and 
using a control horizon $m=6$, a prediction horizon $p=60$ (10 hours) and $T_{S L 1}$ and $T_{S L 2}$ equal to unitary vectors multiplied by scalar 5 . The weights $T_{\text {lower }}$ and $T_{\text {upper }}$ are unitary vectors when variables are outside their control zones and null when they are within. The MVs in table 8 (only final values are shown) were found to be the best available for each plant:

\begin{tabular}{|c|c|c|c|c|c|c|c|c|c|}
\hline \multirow{2}{*}{$u_{1}$} & \multirow{2}{*}{$\begin{array}{c}\text { Manipulated Variables } \\
\text { Description }\end{array}$} & \multirow[t]{2}{*}{ Unit } & \multirow{2}{*}{$\begin{array}{l}\text { Max. } \\
\text { Value }\end{array}$} & \multirow{2}{*}{$\begin{array}{l}\text { Min. } \\
\text { Value }\end{array}$} & \multirow{2}{*}{$\begin{array}{l}\text { Initial } \\
\text { Value }\end{array}$} & \multicolumn{4}{|c|}{ Final Value } \\
\hline & & & & & & $\begin{array}{c}\text { Plant } \\
1\end{array}$ & $\begin{array}{c}\text { Plant } \\
2\end{array}$ & $\begin{array}{c}\text { Plant } \\
3\end{array}$ & $\begin{array}{c}\text { Plant } \\
4\end{array}$ \\
\hline$u_{2}$ & Temperature Fired Heater TIC02.SP & $\mathrm{C}$ & 380.0 & 320.0 & 335.13 & 379.99 & 320.00 & 380.00 & 346.06 \\
\hline$u_{3}$ & Light Diesel Output FC02.SP & $\mathrm{m} 3 / \mathrm{h}$ & $270.0(*)$ & 0.0 & 135.75 & 62.00 & 145.03 & 58.69 & 61.22 \\
\hline$u_{5}$ & Medium Crude Flow Rate FC01A.SP & $\mathrm{m} 3 / \mathrm{h}$ & $800(* *)$ & 0.0 & 550.00 & 112.09 & 288.70 & 1.36 & 753.47 \\
\hline$u_{6}$ & Light Crude Flow Rate FC01B.SP & $\mathrm{m} 3 / \mathrm{h}$ & 800 (**) & 0.0 & 250.00 & 0.11 & 511.29 & 11.17 & 0.07 \\
\hline$u_{7}$ & Heavy Crude Flow Rate FC01C.SP & $\mathrm{m} 3 / \mathrm{h}$ & $800(* *)$ & 0.0 & 0.00 & 687.81 & 0.01 & 787.46 & 46.47 \\
\hline
\end{tabular}

Table 8 - Inputs for case without disturbance. $* u_{3}+u_{4} \geq 85 * * u_{5}+u_{6}+u_{7}=800$.

For these sets of process inputs, the cost function values for each plant are provided in table 9. Lower values are better and thus plant 3 presented the best performance, closely followed by plant 1 , while plants 2 and 4 had worse results. The lower values of the index result from larger amount of cheaper heavy crude oil that could be processed in plant 3 and 1. Comparing plants 2 and 4, the latter performed better since it did not require a large proportion of light oil in order to avoid producing off-spec products.

\begin{tabular}{ccccc}
\hline & Plant 1 & Plant 2 & Plant 3 & Plant 4 \\
\hline $\begin{array}{c}\text { Objective } \\
\text { Function Value }\end{array}$ & 5639.5 & 12693.0 & 5516.2 & 8920.14 \\
\hline Table 9 - Cost Function Values for each Plant & - Case 1.
\end{tabular}

Table 10 provides values for the controlled outputs for each plant. In all three cases the algorithm was able to define a set of inputs that guarantee that no output value violated its zone bounds during the transient or at their final values. The highlighted values show which boundary conditions were active for each plant, which were the quality requirements that restricted profitability. 


\begin{tabular}{lccccccccc}
\hline & Controlled Variables Description & \multirow{2}{*}{ Unit } & \multirow{2}{*}{$\begin{array}{c}\text { Min. } \\
\text { Value }\end{array}$} & Max. & Value & Initial & \multicolumn{4}{c}{ Final Value } \\
\cline { 6 - 10 } & & & & Plant & Plant & Plant & Plant \\
$\boldsymbol{y}_{\mathbf{1}}$ & Cetane Index DIESEL & & 46.0 & - & 44.00 & $\mathbf{4 6 . 0 0}$ & $\mathbf{4 6 . 0 0}$ & $\mathbf{4 6 . 0 0}$ & $\mathbf{4 6 . 0 0}$ \\
$\boldsymbol{y}_{\mathbf{2}}$ & Flash Point DIESEL & $\mathrm{C}$ & 55.0 & - & 81.76 & 145.66 & 89.31 & 86.48 & 95.57 \\
$\boldsymbol{y}_{\mathbf{3}}$ & ASTM D86 DIESEL 65\% & $\mathrm{C}$ & 250.0 & - & 288.81 & 309.46 & 301.96 & 307.47 & 305.82 \\
$\boldsymbol{y}_{\mathbf{4}}$ & ASTM D86 DIESEL 85\% & $\mathrm{C}$ & - & 350.0 & 319.42 & 334.71 & 346.00 & 322.23 & 348.18 \\
$\boldsymbol{y}_{\mathbf{5}}$ & ASTM D86 DIESEL 95\% & $\mathrm{C}$ & - & 370.0 & 341.63 & 355.07 & $\mathbf{3 6 9 . 9 4}$ & 339.75 & 355.70 \\
$\boldsymbol{y}_{\mathbf{6}}$ & Freezing Point DIESEL & $\mathrm{C}$ & - & -15.0 & -25.47 & $\mathbf{- 1 5 . 0 0}$ & -18.75 & $\mathbf{- 1 5 . 0 0}$ & $\mathbf{- 1 5 . 0 0}$ \\
$\boldsymbol{y}_{\mathbf{7}}$ & Density (15 C) DIESEL & $\mathrm{kg} / \mathrm{m}^{3}$ & 820.0 & 860.0 & 826.79 & $\mathbf{8 6 0 . 0 0}$ & 822.51 & $\mathbf{8 5 9 . 9 3}$ & 859.11 \\
$\boldsymbol{y}_{\mathbf{8}}$ & ASTM D86 KEROSENE 100\% & $\mathrm{C}$ & - & 300.0 & 251.11 & 242.67 & 253.33 & 243.74 & 292.88 \\
\hline $\boldsymbol{y}_{\mathbf{9}}$ & Flash Point KEROSENE & $\mathrm{C}$ & 38.0 & - & 49.79 & 123.76 & 58.09 & 129.20 & 95.41 \\
$\boldsymbol{y}_{\mathbf{1 0}}$ & Density (15 C) KEROSENE & $\mathrm{kg} / \mathrm{m}^{3}$ & 775.0 & 840.0 & 812.88 & 813.65 & 816.11 & 808.40 & 822.84 \\
$\boldsymbol{y}_{\mathbf{1 1}}$ & Freezing Point KEROSENE & $\mathrm{C}$ & - & -47.0 & -68.38 & -67.26 & -64.10 & -68.95 & -70.63 \\
\hline
\end{tabular}

Table 10 - Output predictions for scenario without disturbance.

\subsection{Scenario 2 - Handling a Measured Disturbance}

In this scenario the process simulations start at the same steady-state and all parameters were kept at the same values as in case 1. However, the slop recycling flow rate, which acts as a measured disturbance, was increased from zero to $90 \mathrm{~m}^{3} / \mathrm{h}$ at time instant $k$, and kept at this value and until $k+p$. The total crude oil flow rate, composed of light, medium and heavy crudes, was decreased from $800 \mathrm{~m}^{3} / \mathrm{h}$ to $710 \mathrm{~m}^{3} / \mathrm{h}$ in order to accommodate the flow of slop, while keeping total volumetric flow constant. As in case 1, the minimum limit for the Cetane Index, controlled variable $y_{1}$, is 46 and the initial value is lower than required. Table 11 presents the inputs for each plant:

\begin{tabular}{|c|c|c|c|c|c|c|c|c|c|}
\hline \multirow[b]{3}{*}{$u_{1}$} & \multirow[b]{2}{*}{$\begin{array}{c}\text { Manipulated Variables } \\
\text { Description }\end{array}$} & \multirow[b]{2}{*}{ Unit } & \multirow[b]{2}{*}{$\begin{array}{l}\text { Max. } \\
\text { Value }\end{array}$} & \multirow[b]{2}{*}{$\begin{array}{l}\text { Min. } \\
\text { Value }\end{array}$} & \multirow[b]{2}{*}{$\begin{array}{l}\text { Initial } \\
\text { Value }\end{array}$} & \multicolumn{4}{|c|}{ Final Value } \\
\hline & & & & & & $\begin{array}{c}\text { Plant } \\
1\end{array}$ & $\begin{array}{c}\text { Plant } \\
2\end{array}$ & $\begin{array}{c}\text { Plant } \\
3\end{array}$ & $\begin{array}{c}\text { Plant } \\
4\end{array}$ \\
\hline & Temperature 01 tray TIC01.SP & $\mathrm{C}$ & 70.0 & 40.0 & 42.64 & 40.00 & 40.00 & 69.99 & 40.00 \\
\hline$u_{2}$ & Temperature Fired Heater TIC02.SP & $\mathrm{C}$ & 380.0 & 320.0 & 335.13 & 379.98 & 320.00 & 380.00 & 332.93 \\
\hline $\boldsymbol{u}_{3}$ & Light Diesel Output FC02.SP & $\mathrm{m}^{3} / \mathrm{h}$ & $270.0(*)$ & 0.0 & 135.75 & 68.31 & 154.92 & 69.10 & 140.21 \\
\hline $\boldsymbol{u}_{4}$ & Heavy Diesel Output FC03.SP & $\mathrm{m}^{3} / \mathrm{h}$ & $65.0(*)$ & 0.0 & 25.71 & 46.70 & 0.03 & 26.56 & 65.00 \\
\hline$u_{5}$ & Medium Crude Flow Rate FC01A.SP & $\mathrm{m}^{3} / \mathrm{h}$ & $800(* *)$ & 0.0 & 550.00 & 0.01 & 23.59 & 92.24 & 603.23 \\
\hline$u_{6}$ & Light Crude Flow Rate FC01B.SP & $\mathrm{m}^{3} / \mathrm{h}$ & $800(* *)$ & 0.0 & 250.00 & 146.46 & 686.41 & 94.96 & 0.03 \\
\hline $\boldsymbol{u}_{7}$ & Heavy Crude Flow Rate FC01C.SP & $\mathrm{m}^{3} / \mathrm{h}$ & $800(* *)$ & 0.0 & 0.00 & 563.53 & $\mathbf{0 . 0 0}$ & 522.81 & 196.73 \\
\hline$d_{1}$ & Slop Oil Feed Recycling Flow Rate & $\mathrm{m}^{3} / \mathrm{h}$ & 0.00 & 0.00 & 0.00 & 90.00 & 90.00 & 90.00 & 90.00 \\
\hline
\end{tabular}

Table 11 - Inputs for specification change case. $* u_{3}+u_{4} \geq 85 * * u_{5}+u_{6}+u_{7}=800$.

The index values for this scenario can be found in table 12. This time plant 4 had the best performance and plant 1 and 3 followed quite closely. Plant 2 had the worst result yet again, with significantly higher index due to its slower dynamics which prevented it from producing properly specified diesel (see table 13). Compared to plants 1 and 3, plant 4 could produce more diesel and for this reason had a better index. 


\begin{tabular}{ccccc}
\hline & Plant 1 & Plant 2 & Plant 3 & Plant 4 \\
\hline $\begin{array}{c}\text { Objective } \\
\text { Function Value }\end{array}$ & 7576.9 & 34718.4 & 7690.1 & 6987.23 \\
\hline Table 12 - Cost Function Values for & each Plant & Case 2
\end{tabular}

Table 12 - Cost Function Values for each Plant - Case 2 .

Direct comparison of index values between cases 1 and 2 should take into account that case 2 involves lower crude oil flow rates. Compared to case 1, the index was $34.35 \%$ higher for plant 1 . Plant 2 had an index $173.52 \%$ higher; plant 3, 39.41\% higher and plant 4, a $21.66 \%$ lower index. Therefore, the performance degradation due to the disturbance was relatively small for plants 1 and 3 , and very significant for plant 2 . The fact that those plants had lower indices confirms the expected decrease in profitability due to the need to meet quality requirements in the presence of slop in the oil feed, leading to higher crude oil acquisition costs (increased demand for expensive light and medium oils). However, plant 4 made good use of the "free" raw material provided by the slop, and its performance actually improved. Concerning the outputs, we can observe in table 13 that the final cetane value, $y_{1}$, for plant 2 was not met even with almost all the feed composed of light oil. This signifies that plant layout 2 cannot handle a slop recycle of $90 \mathrm{~m}^{3} / \mathrm{h}$ without compromising product quality.

\begin{tabular}{|c|c|c|c|c|c|c|c|c|c|}
\hline \multirow[b]{3}{*}{$y_{1}$} & \multirow{3}{*}{$\begin{array}{c}\text { Controlled Variables Description } \\
\text { Cetane Index DIESEL }\end{array}$} & \multirow{3}{*}{ Unit } & \multirow{3}{*}{$\begin{array}{c}\text { Min. } \\
\text { Value } \\
46.0\end{array}$} & \multirow{3}{*}{$\begin{array}{l}\text { Max. } \\
\text { Value } \\
-\end{array}$} & \multirow{3}{*}{$\begin{array}{c}\text { Initial } \\
\text { Value } \\
44.00\end{array}$} & \multicolumn{4}{|c|}{ Final Value } \\
\hline & & & & & & \multirow{2}{*}{$\begin{array}{c}\text { Plant } \\
1 \\
46.02\end{array}$} & \multirow{2}{*}{$\begin{array}{c}\text { Plant } \\
2 \\
45.78\end{array}$} & \multirow{2}{*}{\begin{tabular}{c|} 
Plant \\
$\mathbf{3}$ \\
46.39 \\
\end{tabular}} & \multirow{2}{*}{$\begin{array}{c}\text { Plant } \\
4 \\
46.00\end{array}$} \\
\hline & & & & & & & & & \\
\hline$y_{2}$ & Flash Point DIESEL & $\mathrm{C}$ & 55.0 & - & 81.76 & 115.16 & 85.75 & 89.13 & 89.24 \\
\hline$y_{3}$ & ASTM D86 DIESEL $65 \%$ & $\mathrm{C}$ & 250.0 & - & 288.81 & 309.91 & 280.16 & 308.18 & 330.93 \\
\hline$y_{4}$ & ASTM D86 DIESEL 85\% & $\mathrm{C}$ & - & 350.0 & 319.42 & 335.51 & 345.97 & 324.00 & 350.00 \\
\hline$y_{5}$ & ASTM D86 DIESEL 95\% & $\mathrm{C}$ & - & 370.0 & 341.63 & 359.02 & 370.00 & 345.48 & 339.34 \\
\hline$y_{6}$ & Freezing Point DIESEL & $\mathrm{C}$ & - & -15.0 & -25.47 & -15.00 & -15.00 & -15.00 & -15.16 \\
\hline$y_{7}$ & Density (15 C) DIESEL & $\mathrm{kg} / \mathrm{m}^{3}$ & 820.0 & 860.0 & 826.79 & 859.56 & 821.35 & 858.32 & 840.17 \\
\hline$y_{8}$ & ASTM D86 KEROSENE $100 \%$ & $\mathrm{C}$ & - & 300.0 & 251.11 & 245.71 & 253.36 & 252.58 & 251.60 \\
\hline$y_{9}$ & Flash Point KEROSENE & $\mathrm{C}$ & 38.0 & - & 49.79 & 117.02 & 59.29 & 110.37 & 68.56 \\
\hline$y_{10}$ & Density (15 C) KEROSENE & $\mathrm{kg} / \mathrm{m}^{3}$ & 775.0 & 840.0 & 812.88 & 816.13 & 818.29 & 810.28 & 819.23 \\
\hline$y_{11}$ & Freezing Point KEROSENE & $\mathrm{C}$ & - & -47.0 & -68.38 & -68.31 & -66.62 & -69.55 & -63.76 \\
\hline
\end{tabular}

Table 13 - Results for specification change case.

\subsection{Selecting the Best Plant}

The results obtained in both scenarios must be considered together in order to draw a consistent conclusion about which plant has the better characteristics when it comes to MPC zone control. Thus an average index will now be defined. It isn't necessary that all components have the same weight in the averaged index. If for instance the chosen 
plant will be frequently required to recycle slop, case 2 should be given greater prominence in the decision process. In table 14, where the average index is presented, case 2 was given double the weight in comparison to case 1 . The average index used in this section was defined as the geometric mean of each plant's indices multiplied by the weight for each scenario.

\begin{tabular}{cccccc}
\hline & Plant 1 & Plant 2 & Plant 3 & Plant 4 & Case Weight \\
\hline Case 1 & 5639.5 & 12693.0 & 5516.2 & 8920.14 & 1 \\
Case 2 & 7576.9 & 34718.4 & 7690.1 & 6987.23 & 2 \\
Geometric Mean & 6866.6 & 24825.3 & 6883.9 & 7579.85 & \\
\hline
\end{tabular}

Table 14 - Average index for each plant.

As can be seen in table 14, plant 1 had the best overall results in this application of the Economic Disturbance Cost Index and should provide better MPC controllability than other. However plant 3's result was very close and it is possible that, in a comprehensive analysis including capital and maintenance costs, either of them may prove a better choice. Plant 2 however is an inadequate design that clearly does not meet requirements due to the very poor choice for the instrument locations which had an adverse impact on model dynamics and control performance. Plant 4 , which is a design that also results in slower dynamics because of its pre-flash drum and additional fired heater, should be avoided as well since it is highly likely to have increased capital costs as well lower performance.

\subsection{Effect of soft landing matrices and error penalization matrices}

The influence of the soft landing matrices in the results, as can be seen from table 15 which presents the rate by which they increase the index, was small.

\begin{tabular}{ccccc}
\hline$\left|\mathbf{S L}_{\mathbf{1}}\right|^{-\mathbf{1}}\left|\mathbf{S L}_{\mathbf{2}}\right|^{\mathbf{- 1}}$ & Plant 1 & Plant 2 & Plant 3 & Plant 4 \\
\hline Case 1 & 1.0067 & 1.0048 & 1.0074 & 1.0070 \\
Case 2 & 1.0069 & 1.0048 & 1.0074 & 1.0072 \\
\hline
\end{tabular}

Table 15 - Effect of SL matrices

This is as expected because the system is stable and the prediction horizon is sufficiently large. However, if not enough time is given to the plant to settle, the impact of $\mathrm{SL}_{1}$ and $\mathrm{SL}_{2}$ may be significant and the solutions will be greatly penalized. Figure 5 shows the index increase for smaller values of $p$ : 


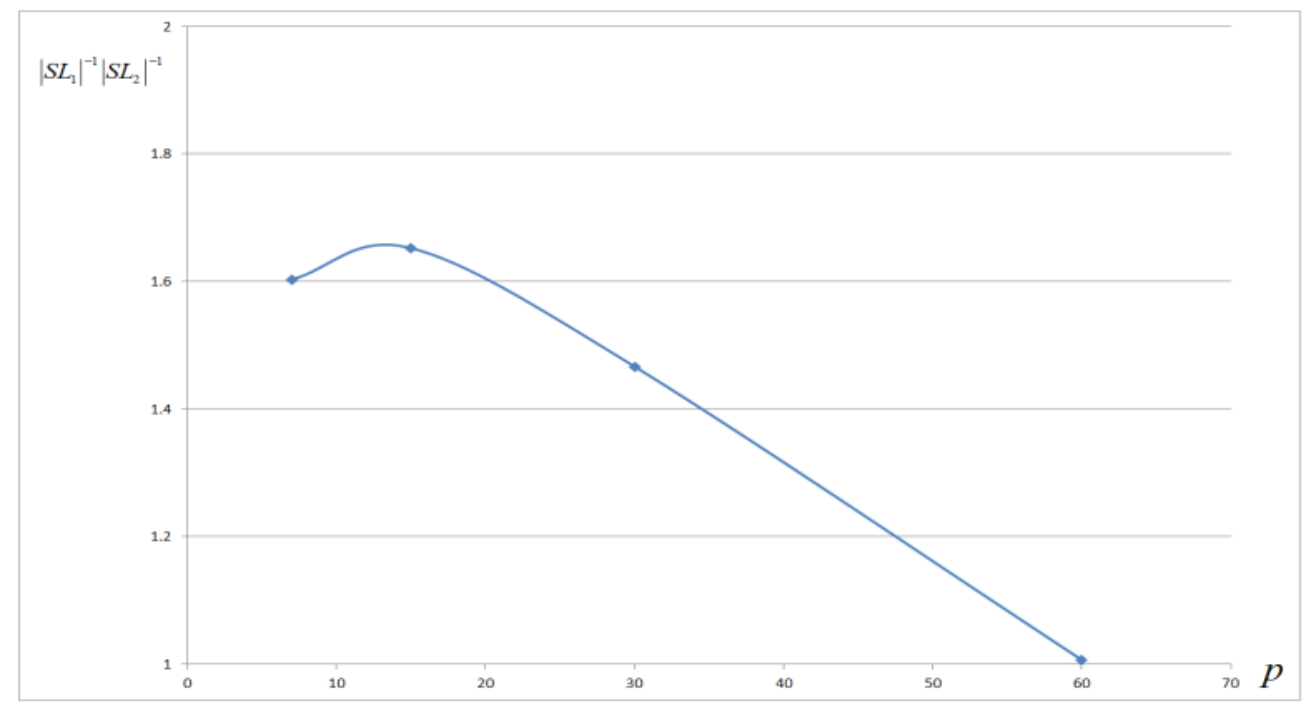

Figure $5-\left|\mathrm{SL}_{1}\right|^{-1}\left|\mathrm{SL}_{2}\right|^{-1}$ for plant 1 , scenario 1 , for various values of $p$.

Since this work is a steady-state focused analysis, the inclusion of SL matrices can be considered a cautionary measure to ensure validity of the results and force the optimization algorithm to disregard oscillatory or overshooting solutions if possible. However, in some design cases the speed of dynamic response may be key and thus it may be necessary to assess the system thoroughly by testing several different values for $p$.

The identity matrix was always used for the error penalization matrix - which means no bound violations are permitted during the transients - with the exception of plant 2 in scenario 2 where $|E P|^{-1}=1.0104$ to allow $y_{1}<y_{\min , 1}$ over the whole predicted range.

\section{Quantifying the Effects of Model Uncertainty}

When designing control systems for robust performance, it is necessary to address model uncertainty. Similarly, when performing controllability analysis the evaluation of plant performance should be robust to uncertainty in the model parameters. If the flowsheets being assessed have very different uncertainty levels in their models, conducting integrated design and control analysis using only the nominal model will eventually lead to wrong conclusions.

In this section model uncertainty is embedded in the Economic MPC Optimisation Index. As discussed in section 3.2 and also in the appendix, the state-space model used in this paper is derived from transfer function models identified from process simulation 
data. Thus, the state-space model of each CV/MV pair inherited the same level of uncertainty of the transfer function from which it is derived.

We shall now define a model uncertainty measure suitable to represent process nonlinearity. Since simulation is the source of process data in the current work, and for this reason there are no sensor related issues, model nonlinearity is the major source of modelling error (numerical error being the remaining possibility). Since step tests are the chosen method of system identification, we are interested in knowing the magnitude of uncertainty in the steady-state output change in response to a control action, as shown in figure 6:

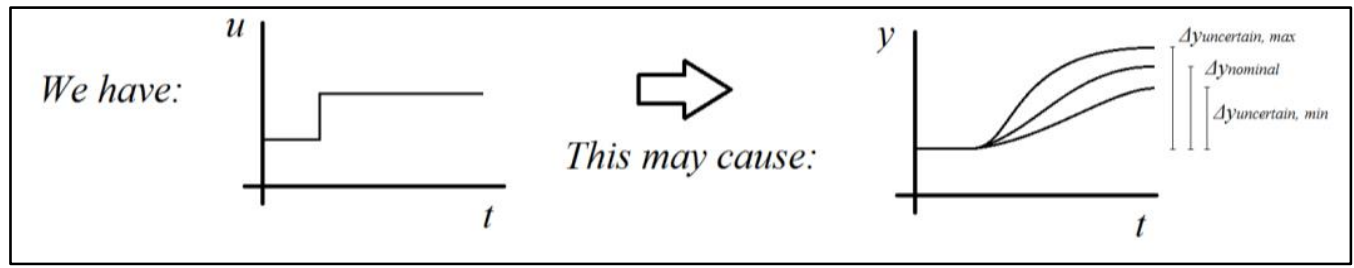

Figure 6 - Uncertainty in the magnitude of the output steady-state change, given a certain input change.

Let us assume that, for each input/output pair, the test used to identify the linear model consists of a series of $n=1, \ldots, N_{s}$ steps. After the nominal model is identified, we may validate it by comparing its response to the identification data, thereby obtaining the model mismatch relative to each one of the steps performed. The model mismatch value can be positive or negative, and so can step response amplitude. The relative model mismatch of the $n^{\text {th }}$ step, $\varphi_{n}$, which relates absolute mismatch to response amplitude, can be obtained using equation 23 :

$$
\varphi_{n}=\left(\Delta y_{s_{n}}-\Delta y_{n o n, s_{n}}\right) / \Delta y_{s_{n}}=e_{s_{n}} / \Delta y_{s_{n}}
$$

where $\Delta y_{s_{n}}$ is the steady-state response amplitude of plant data relative to the $n^{\text {th }}$ step, $\Delta y_{\text {non }, s_{n}}$ is the steady-state response amplitude of the nominal model relative to the $n^{\text {th }}$ step, and $e_{s_{i}}$ is the steady-state absolute value of model mismatch, also relative to the $n^{\text {th }}$ step. Figure 7 illustrates this concept for a model identification test consisting of $N_{s}=$ 


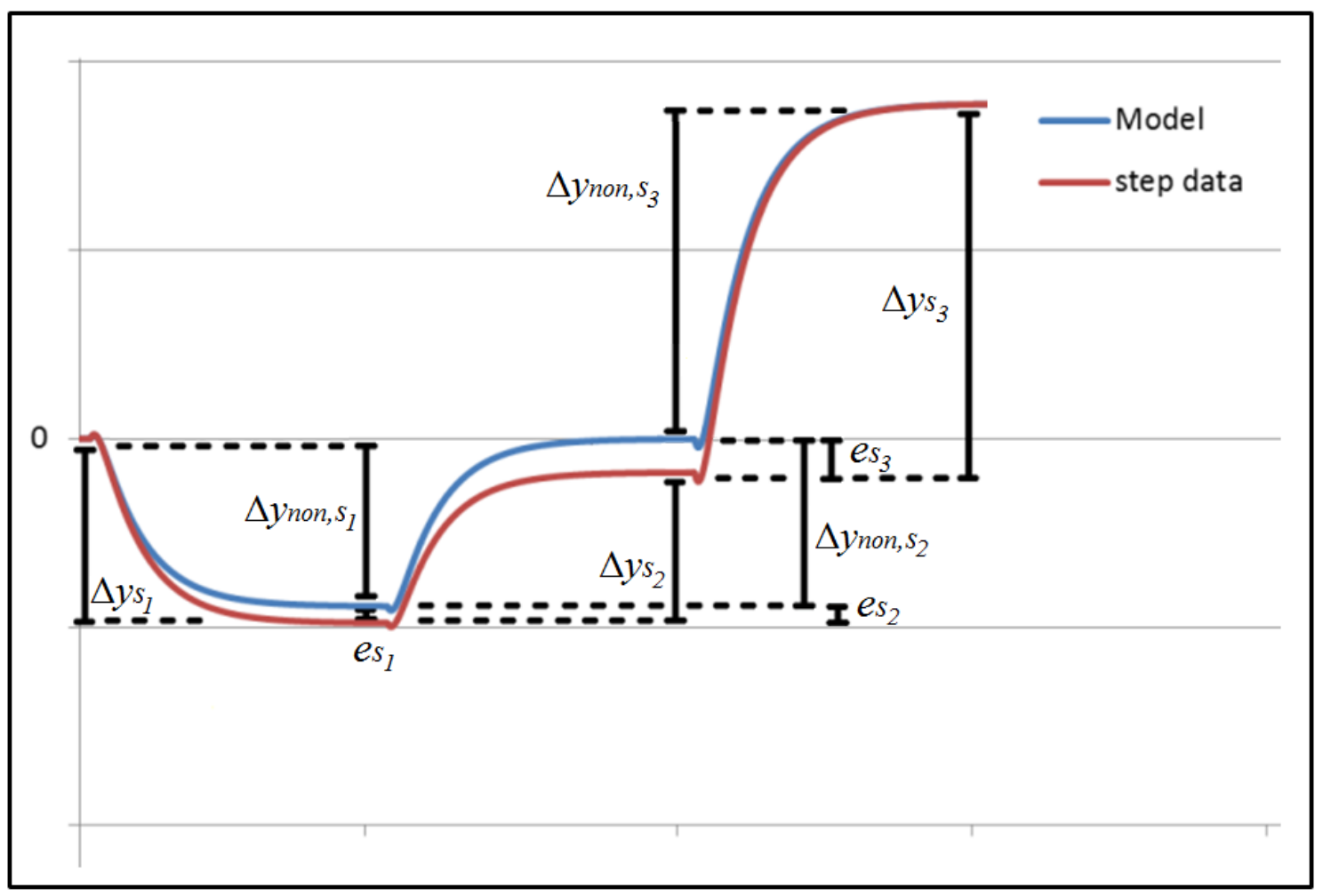

Figure 7 - Representation of model mismatch for a step test.

It is important to consider that, if the output/input pair is weakly related, the denominator in equation 23 is small and $\varphi$ might become too large $(>1)$. In this case the control engineer should ignore uncertainty for this $y / u$ pair and set $\varphi_{n}=0$ for $n=$ $1, \ldots, N_{s}$. It is important to notice that models can be biased to a particular direction, e.g., providing always smaller changes in output prediction than plant data, consistently resulting in negative model mismatch. Or, alternatively, prediction error may be randomly distributed, with a similar number of steps with positive and negative model mismatch. For this reason, equations 24 and 25 define $\beta_{y_{i}, u_{j}}^{-}$and $\beta_{y_{i}, u_{j}}^{+}$, respectively the minimum negative relative model mismatch and the maximum positive relative model mismatch between $y_{i}$ and $u_{j}$ :

$$
\begin{aligned}
& \beta_{y_{i}, u_{j}}^{-}=\min _{n=1, \ldots, N_{s}} \mu_{n} \varphi_{n}, \quad i=1, \ldots, n y, j=1, \ldots, n u \\
& \beta_{y_{i}, u_{j}}^{+}=\max _{n=1, \ldots, N_{s}} \vartheta_{n} \varphi_{n}, \quad i=1, \ldots, n y, j=1, \ldots, n u
\end{aligned}
$$

where $\mu_{n}=1$ and $\vartheta_{n}=0$ if $\varphi_{n}<0, \mu_{n}=0$ and $\vartheta_{n}=1$ if $\varphi_{n}>0, N_{s}$ is again the number of steps, and $n y$ and $n u$ are respectively the number of CVs and MVs. Parameters $\beta_{y_{i}, u_{j}}^{-}$and $\beta_{y_{i}, u_{j}}^{+}$may be understood as fractions of the nominal model response. Similarly, $\beta_{\mathrm{y}_{\mathrm{i}}, \mathrm{d}_{\mathrm{j}}}^{-}$and $\beta_{\mathrm{y}_{\mathrm{i}}, \mathrm{d}_{\mathrm{j}}}^{+}$are respectively the minimum negative and the 
maximum positive relative model mismatch between $y_{i}$ and the disturbance $d_{j}$, as defined in equations 26 and 27 :

$$
\begin{aligned}
& \beta_{y_{i}, d_{j}}^{-}=\min _{n=1, \ldots, N_{s}} \mu_{n} \varphi_{n}, \quad i=1, \ldots, n y, j=1, \ldots, n d \\
& \beta_{y_{i}, d_{j}}^{+}=\max _{n=1, \ldots, N_{s}} \vartheta_{n} \varphi_{n}, \quad i=1, \ldots, n y, j=1, \ldots, n d
\end{aligned}
$$

where $n d$ is the number of disturbances. The control engineer should test several operating points throughout the control zone and in the vicinity using different step amplitudes in order to obtain reliable models and also to ensure that the magnitude of the resulting uncertainty parameter is representative of nonlinearity effects. Let us define a set of matrices in equation $28, B_{u}^{+}, B_{u}^{-}, B_{d}^{+}$and $B_{d}^{-}$, which are going to store all model mismatch data from step tests obtained using equations 24 to 27 for each possible coupling of $y / u$ and $y / d$ :

$B_{u}^{+}=\left[\begin{array}{ccc}\beta_{y_{1}, u_{1}}^{+} & \cdots & \beta_{y_{n y}, u_{1}}^{+} \\ \vdots & \ddots & \vdots \\ \beta_{y_{1}, u_{n u}}^{+} & \cdots & \beta_{y_{n y}, u_{n u}}^{+}\end{array}\right], \quad B_{u}^{-}=\left[\begin{array}{ccc}\beta_{y_{1}, u_{1}}^{-} & \cdots & \beta_{y_{n y}, u_{1}}^{-} \\ \vdots & \ddots & \vdots \\ \beta_{y_{1}, u_{n u}}^{-} & \cdots & \beta_{y_{n y}, u_{n u}}^{-}\end{array}\right]$,

$B_{d}^{+}=\left[\begin{array}{ccc}\beta_{y_{1}, d_{1}}^{+} & \cdots & \beta_{y_{n y}, d_{1}}^{+} \\ \vdots & \ddots & \vdots \\ \beta_{y_{1}, d_{n d}}^{+} & \cdots & \beta_{y_{n y}, d_{n d}}^{+}\end{array}\right], B_{d}^{-}=\left[\begin{array}{ccc}\beta_{y_{1}, d_{1}}^{-} & \cdots & \beta_{y_{n y}, d_{1}}^{-} \\ \vdots & \ddots & \vdots \\ \beta_{y_{1}, d_{n d}}^{-} & \cdots & \beta_{y_{n y}, d_{n d}}^{-}\end{array}\right]$

The uncertainty related to an output change, $\Delta y_{i}$, which arises due to an input movement, $\Delta u_{j}$, may be characterised by the model mismatch. The steady-state output change prediction of the uncertain model, $\Delta y_{i}^{\prime}$, is contained inside the interval defined by the nominal model prediction, $\Delta y_{i}$, multiplied by $1+\beta_{y_{i}, u_{j}}^{-}$and $1+\beta_{y_{i}, u_{j}}^{+}$, as shown in equation 29:

$$
\Delta y_{i}^{u} \in\left[\Delta y_{i} \cdot\left(1+\beta_{y_{i}, u_{j}}^{-}\right), \Delta y_{i} \cdot\left(1+\beta_{y_{i}, u_{j}}^{+}\right)\right], i=1, \ldots, n y, j=1, \ldots, n u
$$

\section{Likewise, we have for disturbances:}

$$
\Delta y_{i}^{d} \in\left[\Delta y_{i} \cdot\left(1+\beta_{y_{i}, d_{j}}^{-}\right), \Delta y_{i} \cdot\left(1+\beta_{y_{i}, d_{j}}^{+}\right)\right], i=1, \ldots, n y, j=1, \ldots, \text { nd }
$$

For the calculation procedure presented in the section, it is also useful to define the uncertainty related to input changes. For instance, imagine we are able to measure plant output and we observe a certain dynamic response, but for some reason the input causing the change in the process cannot be measured. We may use the nominal plant model to estimate the unknown magnitude of the MV (or disturbance) change, which will 
be contained in the value interval $\Delta u_{j}^{\prime}$ (or $\Delta d_{j}^{\prime}$ ), in a similar way to $\Delta y_{i}^{\prime}$. This concept is shown in figure 8:

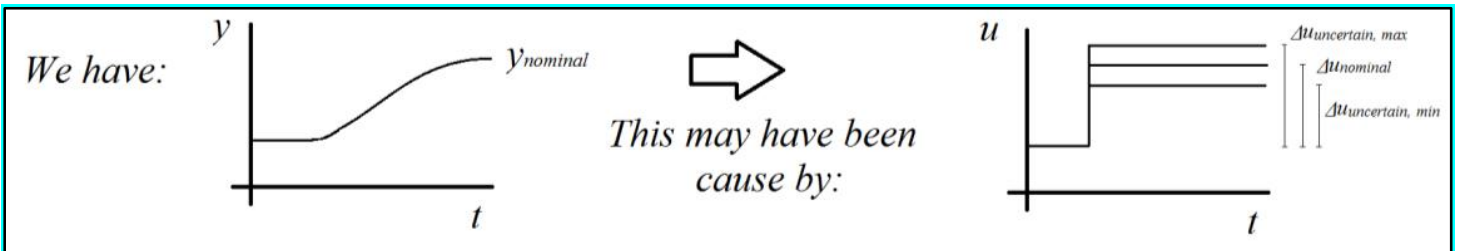

Figure 8 - Uncertainty in the magnitude of an unknown input change, given a certain output change.

Now this concept of uncertainty is clearly defined and we have the understanding that it describes input and output rates of change and not absolute values. Given this uncertainty definition, if a linear continuous-time state-space model is being used, there is a relation of proportionality between $\Delta \mathrm{y}_{\mathrm{i}}^{\mathrm{u}} / \Delta \mathrm{y}_{\mathrm{i}}$ and $\Delta \mathrm{u}_{\mathrm{j}}^{\prime} / \Delta \mathrm{u}_{\mathrm{j}}$, and also between $\Delta \mathrm{y}_{\mathrm{i}}^{\mathrm{d}} / \Delta \mathrm{y}_{\mathrm{i}}$ and $\Delta \mathrm{d}_{\mathrm{j}}^{\prime} / \Delta \mathrm{d}_{\mathrm{j}}$.

This proportionality also applies to the bound definition of uncertainty intervals (equations 29 and 30). Due to model linearity, if we multiply an MV change, $\Delta u_{j}$, by any real number contained in $\left[1+\beta_{y_{i}, u_{j}}^{-}, 1+\beta_{y_{i}, u_{j}}^{+}\right]$, the resulting output change will be contained inside $\Delta y_{i}^{u}$. Similarly, multiplying a disturbance change, $\Delta d_{j}$, by any real number contained in $\left[1+\beta_{y_{i}, d_{j}}^{-}, 1+\beta_{y_{i}, d_{j}}^{+}\right]$, the resulting output change will be contained inside $\Delta y_{i}^{d}$. We use this effect to obtain equations 31 and 32, which are respectively the interval of control action magnitude of the uncertain model, $\Delta u_{j}^{\prime}$, and the interval of disturbance magnitude of the uncertain model, $\Delta d_{j}^{\prime}$ :

$$
\begin{aligned}
& \Delta u_{j}^{\prime} \in\left[\Delta u_{j} \cdot\left(1+\beta_{y_{i}, u_{j}}^{-}\right), \Delta u_{j} \cdot\left(1+\beta_{y_{i}, u_{j}}^{+}\right)\right], i=1, \ldots, n y, j=1, \ldots, n u \\
& \Delta d_{j}^{\prime} \in\left[\Delta d_{j} \cdot\left(1+\beta_{y_{i}, d_{j}}^{-}\right), \Delta d_{i} \cdot\left(1+\beta_{y_{i}, d_{j}}^{+}\right)\right], i=1, \ldots, n y, j=1, \ldots, n d
\end{aligned}
$$

Let us define two new variables, the uncertainty parameters $\gamma_{y_{i}, u_{j}}$ and $\gamma_{y_{i}, d_{j}}$, which are real numbers that can assume any value inside the limits defined by, respectively, $\left[1+\beta_{y_{i}, u_{j}}^{-}, 1+\beta_{y_{i}, u_{j}}^{+}\right]$and $\left[1+\beta_{y_{i}, d_{j}}^{-}, 1+\beta_{y_{i}, d_{j}}^{+}\right]$, as shown in equations 33 and 34 :

$$
\begin{aligned}
& \gamma_{y_{i} u_{j}} \in\left[1+\beta_{y_{i}, u_{j}}^{-}, 1+\beta_{y_{i}, u_{j}}^{+}\right] \\
& \gamma_{y_{i} d_{j}} \in\left[1+\beta_{y_{i}, d_{j}}^{-}, 1+\beta_{y_{i}, d_{j}}^{+}\right]
\end{aligned}
$$


Equation 3, which calculates the output prediction of the nominal model, can be modified to incorporate these new variables, obtaining new output trajectories as the uncertainty parameters assume different values. In order to do that, instead of applying the original MV change $\Delta u_{k}$ at time $k$, we apply $\gamma_{y_{i} u_{j}} \Delta u_{k}$, where $i=1, \ldots n y, j=1, \ldots n u$. At time $\mathrm{k}+1$ we apply $\gamma_{\mathrm{y}_{\mathrm{i}} \mathrm{u}_{\mathrm{j}}} \Delta \mathrm{u}_{\mathrm{k}+1}$ instead of $\Delta \mathrm{u}_{\mathrm{k}+1}$, and so forth until the end of the control horizon, $\mathrm{k}+\mathrm{m}$. The same approach can be used for disturbances. We replace $\Delta \mathrm{d}_{\mathrm{k}}$ for $\gamma_{\mathrm{y}_{\mathrm{i}} \mathrm{d}_{\mathrm{j}}} \Delta \mathrm{d}_{\mathrm{k}}$ at time $\mathrm{k}$, where $\mathrm{i}=1, \ldots \mathrm{ny}, \mathrm{j}=1, \ldots \mathrm{nd}$, and then replace $\Delta \mathrm{d}_{\mathrm{k}+1}$ for $\gamma_{\mathrm{y}_{\mathrm{i}} \mathrm{d}_{\mathrm{j}}} \Delta \mathrm{d}_{\mathrm{k}+1}$ at time $\mathrm{k}+1$, and proceed likewise all the way until the end of the prediction horizon, $\mathrm{k}+\mathrm{p}$. These substitutions yield equation 35 , enabling us to calculate a new output prediction of the uncertain model, $\mathrm{y}^{\prime}$, where $\mathrm{y}^{\prime} \in\left(\Delta \mathrm{y}^{\mathrm{u}} \cup \Delta \mathrm{y}^{\mathrm{d}}\right)$.

$$
\begin{aligned}
& y_{k+1}^{\prime}=C x_{k+1}=C A x_{k}+\operatorname{diag}\left(C B \Gamma_{\Delta u_{k}}\right)+\operatorname{diag}\left(C B \Gamma_{\Delta d_{k}}\right) \\
& y_{k+2}^{\prime}=C x_{k+2}=C A x_{k+1}+\operatorname{diag}\left(C B \Gamma_{\Delta u_{k+1}}\right)+\operatorname{diag}\left(C B \Gamma_{\Delta d_{k+1}}\right) \\
& =C A^{2} x_{k}+\operatorname{diag}\left(\left[\begin{array}{ll}
C A B & C B
\end{array}\right]\left[\begin{array}{c}
\Gamma_{\Delta u_{k}} \\
\Gamma_{\Delta u_{k+1}}
\end{array}\right]\right)+\operatorname{diag}\left(\left[\begin{array}{lll}
C A D & C D
\end{array}\right]\left[\begin{array}{c}
\Gamma_{\Delta d_{k}} \\
\Gamma_{\Delta d_{k+1}}
\end{array}\right]\right) \\
& y_{k+p}^{\prime}=C A^{p} x_{k}+\operatorname{diag}\left(\left[\begin{array}{lllll}
C A^{p-1} B & C A^{p-2} B & \ldots & C A^{p-m} B
\end{array}\right]\left[\begin{array}{lll}
\Gamma_{\Delta u_{k}} & \Gamma_{\Delta u_{k+1}} \ldots \Gamma_{\Delta u_{k+m-1}}
\end{array}\right]^{T}\right) \\
& +\operatorname{diag}\left(\left[\begin{array}{lllll}
C A^{p-1} D & C A^{p-2} D & \ldots & C A^{2} D
\end{array}\right]\left[\begin{array}{llll}
\Gamma_{\Delta d_{k}} & \Gamma_{\Delta d_{k+1}} & \ldots & \Gamma_{\Delta d_{k+p-3}}
\end{array}\right]^{T}\right)
\end{aligned}
$$

where:

$$
\begin{aligned}
\Gamma_{\Delta u_{k+\sigma}, \sigma=0, \ldots, m-1} & =\left[\begin{array}{cccc}
\gamma_{y_{1} u_{1}} \Delta u_{k+\sigma, 1} & \gamma_{y_{2} u_{1}} \Delta u_{k+\sigma, 1} & \cdots & \gamma_{y_{n y} u_{1}} \Delta u_{k+\sigma, 1} \\
\gamma_{y_{1} u_{2}} \Delta u_{k+\sigma, 2} & \gamma_{y_{2} u_{2}} \Delta u_{k+\sigma, 2} & \cdots & \gamma_{y_{n y} u_{2}} \Delta u_{k+\sigma, 2} \\
\vdots & \vdots & \ddots & \vdots \\
\gamma_{y_{1} u_{n u}} \Delta u_{k+\sigma, n u} & \gamma_{y_{2} u_{n u}} \Delta u_{k+\sigma, n u} & \cdots & \gamma_{y_{n y} u_{n u}} \Delta u_{k+\sigma, n u}
\end{array}\right] \\
\Gamma_{\Delta d_{k+\sigma}, \sigma=0, \ldots, p-3} & {\left[\begin{array}{cccc}
\gamma_{y_{1} d_{1}} \Delta d_{k+\sigma, 1} & \gamma_{y_{2} d_{1}} \Delta d_{k+\sigma, 1} & \cdots & \gamma_{y_{n y} d_{1}} \Delta d_{k+\sigma, 1} \\
\gamma_{y_{1} d_{2}} \Delta d_{k+\sigma, 2} & \gamma_{y_{2} d_{2}} \Delta d_{k+\sigma, 2} & \cdots & \gamma_{y_{n y} d_{2}} \Delta d_{k+\sigma, 2} \\
\vdots & \vdots & \ddots & \vdots \\
\gamma_{y_{1} d_{n d}} \Delta d_{k+\sigma, n d} & \gamma_{y_{2} d_{n d}} \Delta d_{k+\sigma, n d} & \cdots & \gamma_{y_{n y} d_{n d}} \Delta d_{k+\sigma, n d}
\end{array}\right] }
\end{aligned}
$$

The Economic MPC Optimisation Index procedure will be now updated to incorporate model uncertainty. Since equation 35 may be used to obtain the feasible intervals of predictions, the optimisation problem can be modified to provide a multilayer optimisation problem, enabling us to calculate the "worst" and "best" possible model indices. The first step of the new procedure is to solve the nominal model problem 
described by equation 20, obtaining the optimised output prediction provided by the nominal model. The second step is to use equation 35 to solve two new optimisation problems in which the model uncertainty parameters vary within their intervals (equations 33 and 34) in order to maximise (worst case) or minimise (best case) the index cost function, while keeping the same set of optimised MVs from the first step, thus obtaining new CV values, $y^{\prime}$. Let us define the difference between the uncertain (best and worst cases) and nominal model's prediction as $\Delta y_{B M}^{\prime}=y_{B M}^{\prime}-y$ and $\quad \Delta y_{W M}^{\prime}=y_{W M}^{\prime}-y$, which represents the bounded uncertainty of plant response related to process nonlinearity. The third and final step consists in twice solving the nominal problem again but with two new starting points defined by equation 36 :

$$
y_{k}^{B M}=y_{k}+\Delta y_{B M, k+p}^{\prime}, \quad y_{k}^{W M}=y_{k}+\Delta y_{W M, k+p}^{\prime}
$$

The initial states must also be updated by solving equation 37 :

$$
0=\sum_{z=0}^{p}\left|y_{k}^{B M}-C A^{Z} x_{k}^{B M}\right|, \quad 0=\sum_{z=0}^{p}\left|y_{k}^{W M}-C A^{Z} x_{k}^{W M}\right|
$$

One of the starting states will be more favourable than the one found using equation 20, leading to a lower index value, while the other less favourable, and thus leading to a higher index value. The control engineer may interpret this third step as being the necessary correction in the control actions a MPC would take when perceiving the mismatch between expected and real plant behaviour. The final form of the uncertain model economic MPC optimisation index using this 3 step procedure is given by equations 38 and 39:

$$
\begin{aligned}
& I_{E_{M P C-W M}}=\min _{\Delta u_{K 3}, y_{k}^{W M}}\left\{\max _{\gamma_{y, u}, \gamma_{y, d}, y^{\prime}}\left[\min _{\Delta u_{K 1}, y}\left(|E P|^{-1}\left|S L_{1}\right|^{-1}\left|S L_{2}\right|^{-1} J_{k+p}\right)\right]\right\} \\
& I_{E_{M P C-B M}}=\min _{\Delta u_{K 3}, y_{k}^{B M}}\left\{\min _{\gamma_{y, u^{\prime}}, \gamma_{y, d}, y^{\prime}}\left[\min _{\Delta u_{K 1}, y}\left(|E P|^{-1}\left|S L_{1}\right|^{-1}\left|S L_{2}\right|^{-1} J_{k+p}\right)\right]\right\}
\end{aligned}
$$

where $\Delta u_{K 1} \in U, \Delta u_{K 3} \in U, \gamma_{y, u} \in\left[I_{n y, n u}+\beta_{y, u}^{-}, I_{n y, n u}+\beta_{y, u}^{+}\right], \gamma_{y, d} \in\left[I_{n y, n d}+\right.$ $\left.\beta_{y, d}^{-}, I_{n y, n d}+\beta_{y, d}^{+}\right]$. The difference between the nominal model index value, found at step 1 , and the value determined using equation 38 represents the increased control effort required for the worst model case. The lower control effort required by the best model case, provided by equation 39, reduces the index since the new starting point provides additional degrees of freedom for economic optimisation. The nominal model value is expected to be contained within the interval established by the best and worst cases which 
are the limiting cases representing the largest possible performance deviation from the nominal model. While the shape of the distribution function is not known for the model parameters, it is likely that the real model is much closer to the nominal model than to the extreme best and worst case models. By incorporating model uncertainty, the analysis now provides for each flowsheet an index interval instead of a single value, bounding the expected MPC and optimisation performances of each plant.

Since model uncertainty and nonlinearity are closely related, equation 38 provides the worst case scenario which predicts the maximum damage that model nonlinearity effects can cause to the process. This worst case may be either due to a poor plant response or the impossibility of meeting specifications. Similarly, equation 39 provides the maximum eventual benefits that could be brought by nonlinearity effects. The nonlinearity of plant behaviour is bounded if this extended method is used.

Let us use as example plant 2, scenario 2, which had the worst result (due to space limitations model uncertainty will not be evaluated for all cases and flowsheets). The uncertainty matrices $B_{u}^{+}, B_{u}^{-}, B_{d}^{+}$and $B_{d}^{-}$for this flowsheet can be found in the appendix (tables 27 to 30). Applying the method described in this section it is possible to obtain an index interval inside which the real flowsheet will be contained. Tables 16, 17 and 18 presents the results provided by best and worst flowsheets within model uncertainty limits and how they compare to the nominal model.

\begin{tabular}{cccccc}
\hline Plant 2 & Step 1 & \multicolumn{2}{c}{ Step 2 } & \multicolumn{2}{c}{ Step 3 } \\
\cline { 2 - 6 } Case 2 & Nominal & Worst & Best & Worst & Best \\
& Model & Case & Case & Case & Case \\
$\boldsymbol{y}_{\mathbf{1}}$ & $\mathbf{4 5 . 7 8}$ & $\mathbf{4 3 . 7 6}$ & 47.66 & $\mathbf{4 1 . 6 9}$ & $\mathbf{4 6 . 0 3}$ \\
$\boldsymbol{y}_{\mathbf{2}}$ & 85.75 & 85.82 & 85.84 & 87.27 & 85.73 \\
$\boldsymbol{y}_{\mathbf{3}}$ & 280.16 & 281.29 & 279.46 & 278.01 & 288.35 \\
$\boldsymbol{y}_{\mathbf{4}}$ & 345.97 & $\mathbf{3 6 1 . 8 9}$ & 344.65 & 341.52 & 323.93 \\
$\boldsymbol{y}_{\mathbf{5}}$ & $\mathbf{3 7 0}$ & $\mathbf{3 9 2 . 0 2}$ & $\mathbf{3 7 0 . 0 0}$ & $\mathbf{3 7 0 . 0 0}$ & $\mathbf{3 6 9 . 9 9}$ \\
$\boldsymbol{y}_{\mathbf{6}}$ & $\mathbf{- 1 5}$ & $\mathbf{- 1 1 . 5 0}$ & $\mathbf{- 1 5 . 0 1}$ & $\mathbf{- 1 5 . 0 0}$ & -17.18 \\
$\boldsymbol{y}_{\mathbf{7}}$ & 821.35 & 821.33 & 821.81 & $\mathbf{8 2 0 . 0 9}$ & $\mathbf{8 5 9 . 7 1}$ \\
$\boldsymbol{y}_{\mathbf{8}}$ & 253.36 & 255.44 & 255.18 & 255.08 & 252.17 \\
$\boldsymbol{y}_{\mathbf{9}}$ & 59.29 & 56.27 & 57.58 & 56.38 & 51.00 \\
$\boldsymbol{y}_{\mathbf{1 0}}$ & 818.29 & 818.18 & 818.20 & 821.05 & 820.43 \\
$\boldsymbol{y}_{\mathbf{1 1}}$ & -66.62 & -67.29 & -67.09 & -68.13 & -76.91 \\
\hline \multicolumn{2}{|c}{ Table $16-$ Uncertain models' CVs. }
\end{tabular}




\begin{tabular}{cccc}
\hline Plant 2 & Step 1/2 & \multicolumn{2}{c}{ Step 3 } \\
\cline { 2 - 4 } Case 2 & $\begin{array}{c}\text { Nominal } \\
\text { Model }\end{array}$ & $\begin{array}{c}\text { Worst } \\
\text { Case }\end{array}$ & $\begin{array}{c}\text { Best } \\
\text { Case }\end{array}$ \\
$\boldsymbol{u}_{\mathbf{1}}$ & $\mathbf{4 0 . 0 0}$ & $\mathbf{4 0 . 0 0}$ & 67.94 \\
$\boldsymbol{u}_{\mathbf{2}}$ & $\mathbf{3 2 0 . 0 0}$ & $\mathbf{3 2 0 . 0 0}$ & 332.24 \\
$\boldsymbol{u}_{\mathbf{3}}$ & 154.92 & 170.02 & 72.77 \\
$\boldsymbol{u}_{\mathbf{4}}$ & $\mathbf{0 . 0 3}$ & 12.29 & 59.91 \\
$\boldsymbol{u}_{\mathbf{5}}$ & 23.59 & 52.76 & 261.59 \\
$\boldsymbol{u}_{\mathbf{6}}$ & 686.41 & 657.24 & 434.29 \\
$\boldsymbol{u}_{\mathbf{7}}$ & $\mathbf{0 . 0 0}$ & $\mathbf{0 . 0 0}$ & 14.11 \\
$\boldsymbol{d}_{\mathbf{1}}$ & $\mathbf{9 0 . 0 0}$ & $\mathbf{9 0 . 0 0}$ & $\mathbf{9 0 . 0 0}$ \\
\hline
\end{tabular}

Table 17 - Uncertain models' MVs

\begin{tabular}{cccc}
\hline Plant 2 & $\begin{array}{c}\text { Nominal } \\
\text { Model }\end{array}$ & $\begin{array}{c}\text { Best } \\
\text { Model }\end{array}$ & $\begin{array}{c}\text { Worst } \\
\text { Model }\end{array}$ \\
\hline Case 2 - Step 3 & $34,718.4$ & $16,744.0$ & $4,342,402.4$ \\
\hline
\end{tabular}

Table 18 - Uncertain models' indices

These tables show that inside the range of uncertainty parameters obtained through model identification for plant 2 , there is a best possible model that would be capable of producing diesel within specification, something that the nominal plant 2 is not capable of accomplishing. The worst case plant is much further away from acceptable performance than the nominal plant. For the worst-case plant 2, there is no opportunity for acceptable MPC performance - a MPC package capable of delivering good performance does not exist since it is not achievable for these conditions.

The best and worst case models are situated at the extremes of model uncertainty and thus are highly unlikely, if not impossible, to be true. If the user is willing to adopt a less rigorous approach in order to obtain a more meaningful index interval, one should consider using the standard deviation of negative and positive relative model mismatches to calculate parameters $\beta_{y, u}^{+}, \beta_{y, u}^{-}, \beta_{y, d}^{+}$and $\beta_{y, d}^{-}$instead of respectively minimum and maximum values. The interval thus obtained might enable better comparison between plants.

\section{Conclusions and Future work}

The work presented a new method to assess the expected controllability, control resilience and profitability of chemical plants where zone constrained or traditional model predictive control is to be used. Here we have used models derived from simulation step 
tests to aid practical implementation and hence encourage its use. A new economic cost index which included elements of controller performance has been developed which enables us to compare designs. The index includes constraint violations, enforcing for hard constraints but allowing some leniency where necessary for soft constraints, and smoothness of response. Best and worst case bounds can be obtained to allow for nonlinearity of the system. The approach has been demonstrated on a problem of considerable complexity, a crude distillation system.

When making use of linear models it is assumed that the process is nearly linear, at least locally, otherwise the model would be invalid when the present values for the variables are too distant from the base case where the model parameters were obtained. Some error due to linearisation can be tolerated but it is necessary to assess whether or not it compromises the whole analysis. If the assumption of a linear model derived from process simulation is reasonably valid within the zone constraints of the control problem, it won't compromise the use of the economic MPC optimization index as presented here. However, we have provided a method for quantifying the effects of model nonlinearity and uncertainty on the index and obtaining an index interval that bounds plant performance.

Nonlinearity effects tend to affect the dynamic response of different flowsheets in a similar way. In particular, the final steady state usually moves in the same direction. For instance consider figure 6 where we compare the diesel flash point $\left(y_{2}\right)$ dynamic response for the base and pre-flash plants, following an increase in medium crude oil flow rate $\left(\Delta u_{5}=+20 \mathrm{~m}^{3}\right)$. In case 1 , both plants were processing a heavy mix of crude oil at a high furnace outlet temperature, whereas in case 2 both plants operated with a much lighter oil mix at low outlet temperature. Due to process nonlinearity, the significant change in operating starting point in effect modified by some degree the size of decrease in $y_{2}$. However the changes were in the same direction (a lower decrease in case 2 relative to case 1 for both plants). Since all plants are similarly affected by changes in operating point and the effects of nonlinearity, they produce smaller changes in the final results and the analysis done with linear models should remain valid unless differences in the index's scores are very small. MPC packages in industrial applications make successful use of linear models, showing that most of the time these effects can be ignored for control purposes. 

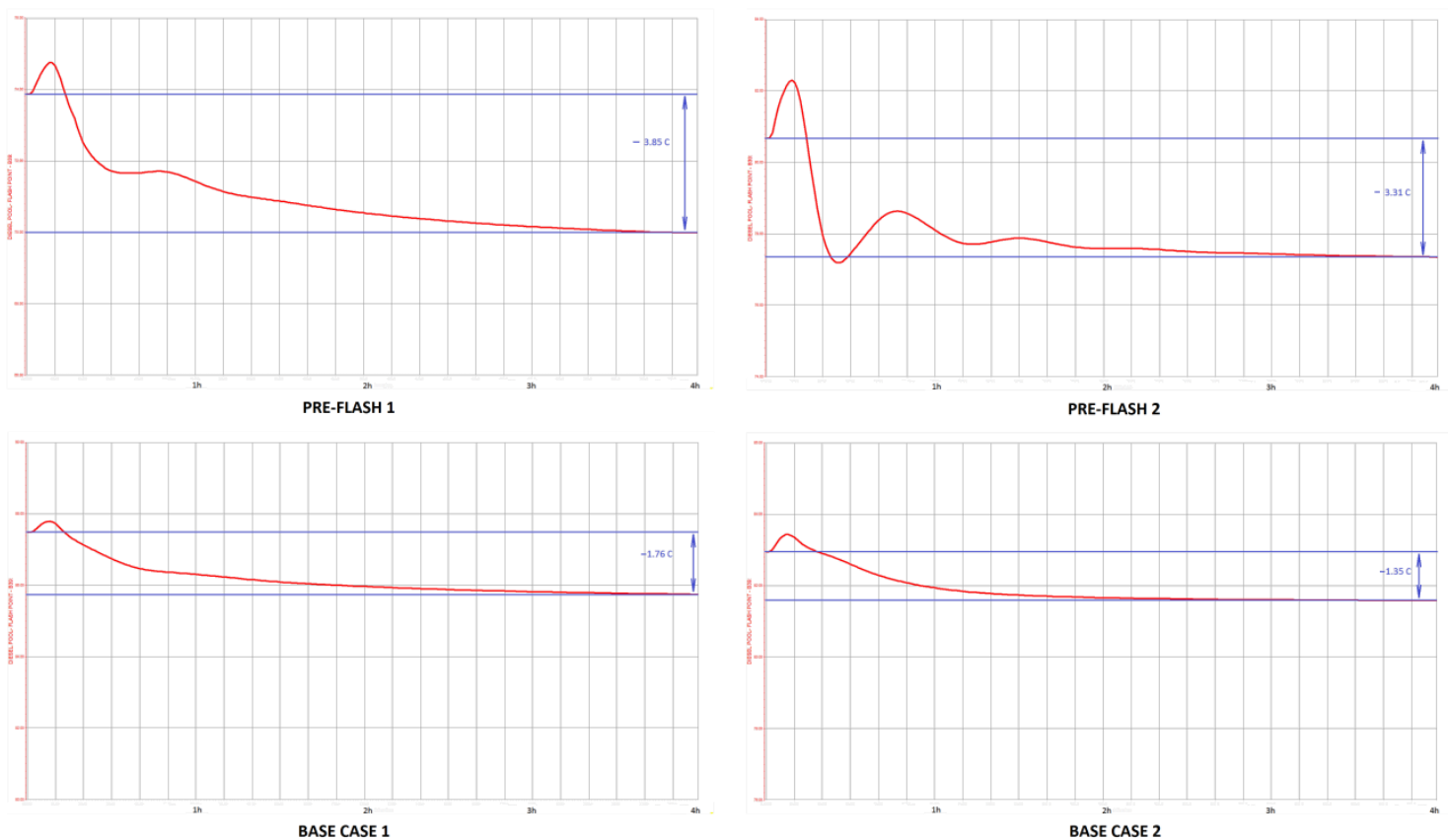

Figure 6 - Comparing nonlinearity for 2 different plants.

Future work will develop a multi-model approach where multiple linear models with different validity zones are used. The use of nonlinear state-space models based on phenomenological models and infinite prediction horizon will also be explored. The downside of these alternatives would likely be the increased computational costs which will reduce the range of processes for which the approach can be applied.

At the time of writing, even when linear models are used, solving the economic MPC optimisation index involves significant computational cost when dealing with complex systems. For example, the problem described in section 3 and 4 was solved using MATLAB® R2014a running on a 3GHz quad-core Intel Xeon E5-1607 CPU, of which $50 \%$ of its capacity was available to the solver. Each iteration takes between 25 and 45 minutes. The computational demand can be much reduced by using smaller control or prediction horizons, $m$ and $p$. Using $m=1$ reduces the computing time necessary to each iteration to only 3-5 minutes. However it is recommend to set $m$ as high as possible, enabling additional degrees of freedom for the algorithm, which in turn leads to less conservative control actions. On the other hand, $k$ only needs to be high enough to compute the whole transient prediction.

\section{Acknowledgement}


The authors thank CAPES and Brazil's Ministry of Education for the financial support and scholarship, and Petrobras and University College London for the encouragement received.

\section{References}

Adetola V, Guay M. Integration of real time optimization and model predictive control. Journal of Process Control 2010;20:125-33.

Alhammadi H, Romagnoli J. Process Design and Operation: Incorporating Environmental, Profitability, Heat Integration and Controllability Considerations. The Integration of Process Design and Control 2004;17:264-306.

Alvarado-Morales M, Abd Hamid MK, Sin G, Gernaey KV, Woodley JM, Gani R. A model-based methodology for simultaneous design and control of a bioethanol production process. Comput Chen Eng 2010;34:2043-61.

Amrit R, Rawlings JB, Biegler LT. Optimizing process economics online using model predictive control. Comput Chem Eng 2013;58:334-43.

Bahakim SS, Ricardez-Sandoval LA. Simultaneous design and MPC-based control for dynamic systems under uncertainty: A stochastic approach. Comput Chem Eng 2014;63:66-81.

Bansal V, Perkins JD, Pistikopoulos EN. A Case Study in Simultaneous Design and Control Using Rigorous, Mixed-Integer Dynamic Optimization Models. Ind Eng Chem Res 2002;41:760-78.

Bansal V, Ross R, Perkins JD, Pistikopoulos EN. The interactions of design and control: double-effect distillation. J Process Contr 2000;10:219-27.

Bemporad A, Morari M. Robust model predictive control: A survey, Lecture Notes in Control and Information Sciences 1999;245:207-226.

Brengel DD, Seider W. Coordinated design and control optimization of nonlinear processes, Chem Eng Com 1992;16:861-86.

Chawankul N, Ricardez-Sandoval LA, Budman H, Douglas PL. Integration of Design and Control: A Robust Control Approach Using MPC. The Canadian Journal of Chemical Engineering 2007;85(4):433-46.

Ferramosca A, Gonzalez AH, Limon D, Odloak D. One-layer robust MPC: a multimodel approach. Preprints of the 19th World Congress, The International Federation of Automatic Control, Cape Town, South Africa. August 24-29, 2014.

Francisco M, Vega P, Alvarez H. Robust integrated design of process with terminal penalty model predictive controllers. Ind Eng Chem Res 2011;89:1011-24.

González AH, Marchetti JL, Odloak D. Extended Robust Model Predictive Control of Integrating Systems. AIChE J 2007;53(7):1758-69.

González AH, Odloak D. A stable MPC with zone control. Journal of Process Control 2009;19(1):110-122.

Gouvêa MT, Odloak D. One-layer real time optimization of LPG production in the FCC unit: Procedure, advantages and disadvantages. Comput Chem Eng 1998;22:191-98. 
Grosman B, Dassau E, Zisser HC, Jovanovič L, Doyle III FJ. Zone Model Predictive Control: A Strategy to Minimize Hyper- and Hypoglycemic Events. Journal of Diabetes Science and Technology 2010;4(4).

Lewin DR. A Simple Tool for Disturbance Resiliency Diagnosis and Feedforward Control Design. Comput Chem Eng 1996; 20:13.

Luo X, Zhou X, Wang S, Xu F. Zone Control with Model Predictive Control for Much Constrained States Process. Proceedings of the 31st Chinese Control Conference, July 25-27, 2012, Hefei, China.

Martins MAF, Yamashita AS, Santoro BF, Odloak D. Robust model predictive control of integrating time delay processes. Journal of Process Control 2013;23:917-32.

Mayne DQ, Rawlings JB, Rao CV, Scokaert POM. Constrained Model Predictive Control: Stability and Optimality. Automatica 2000; 36:789.

Mohideen MJ, Perkins JD, Pistikopoulos EN. Optimal design of dynamic systems under uncertainty. AIChE Journal 1996;42(8):2251-72.

Oliveira MC, Bernussou J, Geromel JC. A new discrete-time robust stability condition. Systems \& Control Letters 1999;37:261-65.

Perkins JD, Walsh SPK. Optimization as a tool for design/control integration. Comput Chem Eng 1996;20(4):315-23.

Porfírio CR, Odloak D. Optimizing model predictive control of an industrial distillation column, Control Engineering Practice 2011;19(10):1137-46.

Ricardez-Sandoval LA, Budman H, Douglas P. Integration of Design and Control for Chemical Processes: A Review of the Literature and Some Recent Results. Annual Reviews in Control 2009;33(2):158-71.

Ricardez-Sandoval LA, Douglas PL, Budman HM. A methodology for the simultaneous design and control of large-scale systems under process parameter uncertainty. Comput Chen Eng 2011;35:307-18.

Rodrigues MA, Odloak D. Output feedback MPC with guaranteed robust stability. Journal of Process Control 2000;10:557-72.

Sakizlis V, Perkins JD, Pistikopoulos EN. Recent advances in optimization-based simultaneous process and control design. Comput Chem Eng 2004;28:2069-86.

Sánchez-Sánchez KB, Ricardez-Sandoval LA. Simultaneous design and control under uncertainty using model predictive control. Ind Eng Chem Res 2013; 52:4815-33.

Sánchez-Sánchez KB, Ricardez-Sandoval LA. Simultaneous process synthesis and control design under uncertainty: A worst-case performance approach. AIChE J 2013;59(7):2497-2514.

Skogestad S, Morari M. Implications of large RGA-elements on control performance. Ind Eng Chem Res 1987:26(11):2323-30.

Solovyev BM, Lewin DR. A Steady-state Process Resiliency Index for Non-linear Processes: 1. Analysis, W. L. Luyben Edition of Ind Eng Chem Res 2002.

Strutzel FAM, Odloak D, Zanin AC. Economic MPC of an Industrial Diesel Hydrotreating Plant. Proceedings of Intelligent Systems and Control (PE 2013), November 11 - 13, 2013, Marina del Rey, USA. 
Trainor M, Giannakeas V, Kiss C, Ricardez-Sandoval, LA. Optimal process and control design under uncertainty: A methodology with robust feasibility and stability analyses. Chem Eng Sci 2013;104:1065-80.

Vidyasagar M. Nonlinear Systems Analysis 2nd edition. Society for Industrial and Applied Mathematics 2002.

Weitz O, Lewin DR. Dynamic controllability and resiliency diagnosis using steady state process flowsheet data. Comput Chem Eng 1996;20(4):325-35.

Yuan Z, Chen B, Zhao J. An overview on controllability analysis of chemical processes. AIChE Journal 2011; 57(5):1185-1201.

Zhang X, Luo X, Wang S. The Expression of Control Law for Zone Constraints Predictive Control. Artificial Intelligence and Computational Intelligence, Second International Conference, AICI 2011, Taiyuan, China, September 24-25, 2011. 


\section{Appendix}

\section{Continuous-Time Transfer Functions for the Crude Oil Distillation Process}

As discussed in section 3.2, this work made use of the output prediction oriented model (OPOM), a state-space formulation that is built upon the transfer functions that define the interaction between each pair of manipulated/disturbance and controlled variable. In order to identify these models, a series of step tests was carried using the dynamic simulations of the four different designs of the crude oil distillation process presented in section 4 . Tables from 14 to 21 display parameters to be substituted in equation 19 as a means to represent each model as a $5^{\text {th }}$ order transfer function.

$$
G_{i, j}(s)=\frac{b_{i, j, 5} s^{5}+b_{i, j, 4} s^{4}+b_{i, j, 3} s^{3}+b_{i, j, 2} s^{2}+b_{i, j, 1} s+b_{i, j, 0}}{a_{i, j, 5} s^{5}+a_{i, j, 4} s^{4}+a_{i, j, 3} s^{3}+a_{i, j, 2} s^{2}+a_{i, j, 1} s+a_{i, j, 0}}
$$


Table 19 - Plant 1 - Transfer Function Numerator Parameters

\begin{tabular}{|c|c|c|c|c|c|c|c|c|c|}
\hline P1 & $\mathrm{N}$ & $\mathrm{u}_{1}$ & $\mathrm{u}_{2}$ & $\mathrm{u}_{3}$ & $\mathrm{u}_{4}$ & $\mathrm{u}_{5}$ & $\mathrm{u}_{6}$ & $\mathrm{u}_{7}$ & $\mathrm{~d}_{1}$ \\
\hline \multirow{6}{*}{$\mathrm{y}_{1}$} & b5 & -0.00105818 & 0.00042034 & 0 & 0 & 0 & 0 & 0 & 0 \\
\hline & b4 & -0.00056843 & -0.00039612 & 0 & 0 & 0 & 0 & 0 & 0 \\
\hline & b3 & 0.00108421 & -0.00003100 & -0.00055424 & 0 & 0 & 0 & 0 & 0 \\
\hline & b2 & 0.00006603 & 0.00010802 & -0.00896867 & 0 & 0 & 0 & 0 & 0 \\
\hline & b1 & 0.00000054 & 0.00000079 & -0.00003071 & 0.02139236 & 0.00121968 & 0.00176262 & 0.00108316 & -0.00034149 \\
\hline & b0 & 0 & 0 & 0.00001280 & 0.00035320 & -0.00012844 & -0.00017392 & -0.00011947 & -0.00000220 \\
\hline \multirow{6}{*}{$y_{2}$} & b5 & 0.00679037 & 0 & 0 & 0 & 0 & 0 & 0 & 0 \\
\hline & b4 & -0.00603944 & 0 & 0 & 0 & 0 & 0 & 0 & 0 \\
\hline & b3 & 0.00170123 & 0.01504257 & 0 & 0 & 0 & 0 & 0 & 0 \\
\hline & b2 & 0.00048806 & -0.01420358 & 0 & 0 & 0 & 0 & 0 & 0 \\
\hline & b1 & 0.00001045 & 0.00442552 & 0.00248820 & 0.03528191 & -0.00224608 & -0.00275145 & -0.00181754 & -0.00179053 \\
\hline & b0 & 0.00000002 & 0.00001929 & 0.00001545 & 0.00050681 & -0.00000431 & -0.00000197 & -0.00000513 & -0.00000782 \\
\hline \multirow{6}{*}{$\mathrm{y}_{3}$} & b5 & 0 & 0 & 0 & 0 & 0 & 0 & 0 & 0 \\
\hline & b4 & 0 & 0 & 0 & -0.00040067 & 0 & 0 & 0 & 0 \\
\hline & b3 & 0.01553926 & 0.02977826 & 0 & 6.86898674 & 0 & 0 & 0 & 0 \\
\hline & b2 & -0.02796074 & -0.02257607 & 0 & 4.24330364 & 0 & 0 & 0 & 0 \\
\hline & b1 & 0.00831718 & 0.00640430 & -0.09726105 & 0.54925112 & 0.02443288 & 0.03043380 & 0.01918137 & -0.00480119 \\
\hline & b0 & 0.00013150 & -0.00006168 & 0.01421374 & 0.00673292 & -0.00236608 & -0.00287664 & -0.00189252 & -0.00001670 \\
\hline \multirow{6}{*}{$\mathrm{y}_{4}$} & b5 & 0 & 0 & 0 & 0 & 0 & 0 & 0 & 0 \\
\hline & b4 & 0.00781357 & 0 & 0 & 0 & 0 & 0 & 0 & 0 \\
\hline & b3 & -0.00926354 & 0.02105311 & 0 & 0 & 0 & 0 & 0 & 0 \\
\hline & b2 & 0.00214191 & -0.01587539 & 0 & 0 & 0 & 0 & 0 & 0 \\
\hline & b1 & 0.00024485 & 0.00425530 & -0.10991325 & 0.15626595 & 0.01542513 & 0.01904798 & 0.01229491 & -0.00371796 \\
\hline & b0 & 0.00000197 & -0.00005474 & 0.01070043 & 0.00297900 & -0.00176067 & -0.00208362 & -0.00143810 & -0.00001546 \\
\hline \multirow{6}{*}{$\mathrm{y}_{5}$} & b5 & 0 & 0 & 0 & 0 & 0 & 0 & 0 & 0 \\
\hline & b4 & 0 & 0 & 0 & 0 & 0 & 0 & 0 & 0 \\
\hline & b3 & 0 & 0 & 0 & 0 & 0 & 0 & 0 & 0 \\
\hline & b2 & -0.00245307 & 0 & 0 & 0 & 0 & 0 & 0 & 0 \\
\hline & b1 & 0.00024504 & 0.01313890 & -0.03147818 & 0.12605222 & 0.00626059 & 0.00755384 & 0.00499517 & -0.00196314 \\
\hline & b0 & 0.00017616 & -0.00018301 & 0.00264057 & 0.00388943 & -0.00061168 & -0.00070769 & -0.00050397 & -0.00013213 \\
\hline \multirow{6}{*}{$\mathrm{y}_{6}$} & b5 & 0.00362844 & 0 & 0 & 0 & 0 & 0 & 0 & 0 \\
\hline & b4 & 0.00391296 & 0 & 0 & 0 & 0 & 0 & 0 & 0 \\
\hline & b3 & -0.00518816 & 0.01556868 & 0 & 0 & 0 & 0 & 0 & 0 \\
\hline & b2 & 0.00187936 & -0.01177573 & 0 & 0 & 0 & 0 & 0 & 0 \\
\hline & b1 & 0.00002980 & 0.00320614 & -0.03571791 & 0.09379727 & 0.01108018 & 0.01385702 & 0.00860859 & -0.00212006 \\
\hline & b0 & 0 & -0.00002768 & 0.00425902 & 0.00710740 & -0.00099387 & -0.00121532 & -0.00078800 & -0.00000685 \\
\hline \multirow{6}{*}{$\mathrm{y}_{7}$} & b5 & 0 & 0 & 0 & 0 & 0 & 0 & 0 & 0 \\
\hline & b4 & 0 & 0 & 0 & 0 & 0 & 0 & 0 & 0 \\
\hline & b3 & 0 & 0.00561809 & 0 & 0 & 0 & 0 & 0 & 0 \\
\hline & b2 & 0 & -0.00410249 & 0 & 0 & 0 & 0 & 0 & 0 \\
\hline & b1 & 0.00315502 & 0.00103698 & -0.26102578 & 0.03456394 & 0.02435825 & 0.02265670 & 0.02866951 & 0.13951032 \\
\hline & b0 & 0.00003672 & -0.00001600 & -0.00244054 & 0.00064642 & -0.00062013 & -0.00069358 & -0.00057060 & -0.00098788 \\
\hline \multirow{6}{*}{$\mathrm{y}_{8}$} & b5 & 0 & 0 & 0 & 0 & 0 & 0 & 0 & 0 \\
\hline & b4 & 0 & 0 & 0 & 0 & 0 & 0 & 0 & 0 \\
\hline & b3 & -0.01984206 & 0 & 0 & 0 & 0 & 0 & 0 & 0 \\
\hline & b2 & 0.01032112 & 0 & 0 & 0 & 0 & 0 & 0 & 0 \\
\hline & b1 & 0.00000003 & 0.00773138 & -0.00014980 & -0.00014347 & 0.00832288 & 0.00964860 & 0.00631374 & -0.00260981 \\
\hline & b0 & 0.00000009 & -0.00015699 & 0.00013360 & 0.00000367 & -0.00087961 & -0.00105437 & -0.00065952 & -0.00001146 \\
\hline \multirow{6}{*}{$\mathrm{y}_{9}$} & b5 & 0 & -0.00059149 & 0 & 0 & 0 & 0 & 0 & 0 \\
\hline & b4 & 0 & 0.00049619 & 0 & 0 & 0 & 0 & 0 & 0 \\
\hline & b3 & -0.00716071 & -0.00026858 & 0 & 0 & 0 & 0 & 0 & 0 \\
\hline & b2 & 0.00377624 & 0.00013675 & 0 & 0 & 0 & 0 & 0 & 0 \\
\hline & b1 & 0.00032277 & -0.00000042 & 0.00008754 & -0.00003582 & -0.00070503 & -0.00092152 & -0.00055914 & -0.00078310 \\
\hline & b0 & 0.00000234 & -0.00000001 & 0.00000182 & 0.00000118 & -0.00000049 & -0.00000018 & -0.00000189 & -0.00000523 \\
\hline \multirow{6}{*}{$\mathrm{y}_{10}$} & b5 & 0 & 0 & 0 & 0 & 0 & 0 & 0 & 0 \\
\hline & b4 & 0 & 0 & 0 & 0 & 0 & 0 & 0 & 0 \\
\hline & b3 & 0 & 0 & 0 & 0 & 0 & 0 & 0 & 0 \\
\hline & b2 & 0 & 0 & 0 & 0 & 0 & 0 & 0 & 0 \\
\hline & b1 & 0.00396175 & 0.00276120 & 0.00005984 & 0.00000856 & 0.00987724 & 0.01017977 & 0.00916876 & -0.00026726 \\
\hline & b0 & 0.00002691 & -0.00006181 & -0.00000001 & -0.00000002 & -0.00071999 & -0.00084555 & -0.00054283 & -0.00000528 \\
\hline \multirow{6}{*}{$\mathrm{y}_{11}$} & b5 & 0 & 0 & 0 & 0 & 0 & 0 & 0 & 0 \\
\hline & b4 & 0 & 0.00015430 & 0 & 0 & 0 & 0 & 0 & 0 \\
\hline & b3 & -0.00179741 & -0.00010968 & 0 & 0 & 0 & 0 & 0 & 0 \\
\hline & b2 & 0.00339504 & 0.00013629 & 0 & 0 & 0 & 0 & 0 & 0 \\
\hline & b1 & 0.00028796 & -0.00000261 & 0.00004086 & -0.00005324 & 0.00287173 & 0.00397110 & 0.00223878 & -0.00077358 \\
\hline & b0 & 0.00000205 & -0.00000006 & 0 & 0.00000205 & -0.00037071 & -0.00051908 & -0.00027936 & -0.00000293 \\
\hline
\end{tabular}


Table 20 - Plant 1 - Transfer Function Denominator Parameters

\begin{tabular}{|c|c|c|c|c|c|c|c|c|c|}
\hline $\mathrm{P} 1$ & D & $u_{1}$ & $u_{2}$ & $u_{3}$ & $u_{4}$ & $u_{5}$ & $u_{6}$ & $u_{7}$ & $d_{1}$ \\
\hline \multirow{6}{*}{$y_{1}$} & a5 & 1 & 1 & 0 & 0 & 0 & 0 & 0 & 0 \\
\hline & $\mathrm{a} 4$ & 1.36366801 & 0.26264684 & 0 & 0 & 0 & 0 & 0 & 0 \\
\hline & a3 & 0.09234154 & 0.04216460 & 1 & 0 & 0 & 0 & 0 & 0 \\
\hline & $\mathrm{a} 2$ & 0.00147621 & 0.00312024 & 0.68167658 & 1 & 1 & 1 & 1 & 1 \\
\hline & a1 & 0.00000680 & 0.00005366 & 0.03560013 & 0.37340324 & 0.31033466 & 0.34452964 & 0.35176847 & 0.02193683 \\
\hline & $\mathrm{a} 0$ & 0 & 0.00000026 & 0.00030782 & 0.00407043 & 0.00322648 & 0.00354395 & 0.00370116 & 0.00007177 \\
\hline \multirow{6}{*}{$y_{2}$} & a5 & 1 & 0 & 0 & 0 & 0 & 0 & 0 & 0 \\
\hline & $\mathrm{a} 4$ & 0.29949751 & 0 & 0 & 0 & 0 & 0 & 0 & 0 \\
\hline & a3 & 0.06488160 & 1 & 0 & 0 & 0 & 0 & 0 & 0 \\
\hline & $\mathrm{a} 2$ & 0.00306087 & 0.14054801 & 1 & 1 & 1 & 1 & 1 & 1 \\
\hline & a1 & 0.00002895 & 0.01763127 & 0.03620743 & 0.43866775 & 0.01528766 & 0.01367732 & 0.01666919 & 0.02230786 \\
\hline & $\mathrm{a} 0$ & 0.00000005 & 0.00022272 & 0.00017480 & 0.00526192 & 0.00002434 & 0.00000847 & 0.00003723 & 0.00005905 \\
\hline \multirow{6}{*}{$y_{3}$} & a5 & 0 & 0 & 0 & 0 & 0 & 0 & 0 & 0 \\
\hline & $\mathrm{a} 4$ & 0 & 0 & 0 & 1 & 0 & 0 & 0 & 0 \\
\hline & a3 & 1 & 1 & 0 & 46.03110338 & 0 & 0 & 0 & 0 \\
\hline & $\mathrm{a} 2$ & 0.37554760 & 0.13570009 & 1 & 17.58709116 & 1 & 1 & 1 & 1 \\
\hline & a1 & 0.02516782 & 0.01664963 & 1.21089344 & 1.56310701 & 0.30891927 & 0.30998539 & 0.30139694 & 0.02815219 \\
\hline & $\mathrm{a} 0$ & 0.00021989 & 0.00035331 & 0.03459826 & 0.01624211 & 0.00785270 & 0.00778083 & 0.00785004 & 0.00007089 \\
\hline \multirow{6}{*}{$y_{4}$} & a5 & 0 & 0 & 0 & 0 & 0 & 0 & 0 & 0 \\
\hline & $\mathrm{a} 4$ & 1 & 0 & 0 & 0 & 0 & 0 & 0 & 0 \\
\hline & a3 & 0.22287789 & 1 & 0 & 0 & 0 & 0 & 0 & 0 \\
\hline & $\mathrm{a} 2$ & 0.02825536 & 0.12988569 & 1 & 1 & 1 & 1 & 1 & 1 \\
\hline & a1 & 0.00057221 & 0.01387728 & 1.13116353 & 0.38330062 & 0.34581491 & 0.33921567 & 0.34447801 & 0.01855660 \\
\hline & $\mathrm{a} 0$ & 0.00000268 & 0.00016161 & 0.01587600 & 0.00436194 & 0.00437167 & 0.00425213 & 0.00442939 & 0.00004917 \\
\hline \multirow{6}{*}{$y_{5}$} & a5 & 0 & 0 & 0 & 0 & 0 & 0 & 0 & 0 \\
\hline & $\mathrm{a} 4$ & 0 & 0 & 0 & 0 & 0 & 0 & 0 & 0 \\
\hline & a3 & 1 & 0 & 0 & 0 & 0 & 0 & 0 & 0 \\
\hline & $\mathrm{a} 2$ & 0.18435735 & 1 & 1 & 1 & 1 & 1 & 1 & 1 \\
\hline & a1 & 0.03079164 & 0.05226808 & 0.36874216 & 0.53383295 & 0.17203977 & 0.16586337 & 0.17312671 & 0.06442452 \\
\hline & $\mathrm{a} 0$ & 0.00024846 & 0.00043811 & 0.00357386 & 0.00508517 & 0.00150286 & 0.00143274 & 0.00153275 & 0.00041912 \\
\hline \multirow{6}{*}{$y_{6}$} & a5 & 1 & 0 & 0 & 0 & 0 & 0 & 0 & 0 \\
\hline & $\mathrm{a} 4$ & 0.37737981 & 0 & 0 & 0 & 0 & 0 & 0 & 0 \\
\hline & a3 & 0.19790672 & 1 & 0 & 0 & 0 & 0 & 0 & 0 \\
\hline & $\mathrm{a} 2$ & 0.01217853 & 0.14981125 & 1 & 1 & 1 & 1 & 1 & 1 \\
\hline & a1 & 0.00012641 & 0.01813079 & 0.93067637 & 0.80316627 & 0.25988499 & 0.26252973 & 0.25255726 & 0.03198192 \\
\hline & $\mathrm{a} 0$ & 0 & 0.00046621 & 0.03645834 & 0.03933381 & 0.00865629 & 0.00860474 & 0.00864419 & 0.00007701 \\
\hline \multirow{6}{*}{$y_{7}$} & a5 & 0 & 0 & 0 & 0 & 0 & 0 & 0 & 0 \\
\hline & $\mathrm{a} 4$ & 0 & 0 & 0 & 0 & 0 & 0 & 0 & 0 \\
\hline & a3 & 0 & 1 & 0 & 0 & 0 & 0 & 0 & 0 \\
\hline & $\mathrm{a} 2$ & 1 & 0.10820676 & 1 & 1 & 1 & 1 & 1 & 1 \\
\hline & a1 & 0.03860094 & 0.01385937 & 0.76285742 & 0.38462990 & 0.62609412 & 0.53592590 & 0.79156012 & 3.68800514 \\
\hline & $\mathrm{a} 0$ & 0.00026153 & 0.00020083 & 0.01086380 & 0.00454312 & 0.01119549 & 0.00949914 & 0.01428156 & 0.04281448 \\
\hline \multirow{6}{*}{$y_{8}$} & a5 & 0 & 0 & 0 & 0 & 0 & 0 & 0 & 0 \\
\hline & a4 & 0 & 0 & 0 & 0 & 0 & 0 & 0 & 0 \\
\hline & a3 & 1 & 0 & 0 & 0 & 0 & 0 & 0 & 0 \\
\hline & $\mathrm{a} 2$ & 0.01125625 & 1 & 1 & 1 & 1 & 1 & 1 & 1 \\
\hline & a1 & 0.00000908 & 0.06213082 & 0.53419340 & 0.04302812 & 0.21492739 & 0.21375936 & 0.21397409 & 0.01718509 \\
\hline & $\mathrm{a} 0$ & 0.00000010 & 0.00063973 & 0.00510207 & 0.00034629 & 0.00225498 & 0.00221746 & 0.00225901 & 0.00004192 \\
\hline \multirow{6}{*}{$y_{9}$} & a5 & 0 & 1 & 0 & 0 & 0 & 0 & 0 & 0 \\
\hline & $\mathrm{a} 4$ & 0 & 0.37650807 & 0 & 0 & 0 & 0 & 0 & 0 \\
\hline & a3 & 1 & 0.06332451 & 0 & 0 & 0 & 0 & 0 & 0 \\
\hline & $\mathrm{a} 2$ & 0.12833073 & 0.00623091 & 1 & 1 & 1 & 1 & 1 & 1 \\
\hline & a1 & 0.00206372 & 0.00012364 & 0.08671226 & 0.16918886 & 0.00749110 & 0.00715718 & 0.01098416 & 0.02361598 \\
\hline & $\mathrm{a} 0$ & 0.00000679 & 0.00000045 & 0.00046283 & 0.00081333 & 0.00000412 & 0.00000056 & 0.00002285 & 0.00006049 \\
\hline \multirow{6}{*}{$y_{10}$} & a5 & 0 & 0 & 0 & 0 & 0 & 0 & 0 & 0 \\
\hline & $\mathrm{a} 4$ & 0 & 0 & 0 & 0 & 0 & 0 & 0 & 0 \\
\hline & a3 & 0 & 0 & 0 & 0 & 0 & 0 & 0 & 0 \\
\hline & $\mathrm{a} 2$ & 1 & 1 & 1 & 1 & 1 & 1 & 1 & 1 \\
\hline & a1 & 0.02159885 & 0.09590390 & 0.01255660 & 0.00102691 & 0.71893254 & 0.66024677 & 0.73703103 & 0.01790781 \\
\hline & $\mathrm{a} 0$ & 0.00008608 & 0.00120329 & 0 & 0 & 0.00755526 & 0.00696963 & 0.00779396 & 0.00007437 \\
\hline \multirow{6}{*}{$y_{11}$} & a5 & 0 & 1 & 0 & 0 & 0 & 0 & 0 & 0 \\
\hline & $\mathrm{a} 4$ & 0 & 0.40106484 & 0 & 0 & 0 & 0 & 0 & 0 \\
\hline & a3 & 1 & 0.06563132 & 0 & 0 & 0 & 0 & 0 & 0 \\
\hline & $\mathrm{a} 2$ & 0.09943133 & 0.00682578 & 1 & 1 & 1 & 1 & 1 & 1 \\
\hline & a1 & 0.00206009 & 0.00019807 & 0.01363191 & 0.08976900 & 0.33146117 & 0.36683151 & 0.32300744 & 0.02239869 \\
\hline & $\mathrm{a} 0$ & 0.00001011 & 0.00000152 & 0.00000111 & 0.00125016 & 0.00542167 & 0.00584097 & 0.00535404 & 0.00005439 \\
\hline
\end{tabular}


Table 21 - Plant 2 - Transfer Function Numerator Parameters

\begin{tabular}{|c|c|c|c|c|c|c|c|c|c|}
\hline P2 & $\mathrm{N}$ & $u_{I}$ & $u_{2}$ & $u_{3}$ & $u_{4}$ & $u_{5}$ & $u_{6}$ & $u_{7}$ & $d_{1}$ \\
\hline \multirow{6}{*}{$y_{1}$} & b5 & -0.00018774 & 0.00147645 & 0 & 0 & 0 & 0 & 0 & 0 \\
\hline & b4 & -0.00000214 & 0.00014399 & 0 & 0 & 0 & 0 & 0 & 0 \\
\hline & b3 & 0.00000166 & 0.00001765 & 0.00006663 & 0 & 0 & 0 & 0 & 0 \\
\hline & b2 & 0 & 0.00000027 & -0.00001160 & 0 & 0 & 0 & 0 & 0 \\
\hline & b1 & 0 & 0 & 0.00000046 & 0.00004259 & 0.00000418 & 0.00000595 & 0.00000450 & -0.00002998 \\
\hline & b0 & 0 & 0 & 0.00000008 & 0.00000087 & -0.00000075 & -0.00000091 & -0.00000060 & -0.00000008 \\
\hline \multirow{6}{*}{$y_{2}$} & b5 & -0.00604111 & 0 & 0 & 0 & 0 & 0 & 0 & 0 \\
\hline & b4 & -0.00055015 & 0 & 0 & 0 & 0 & 0 & 0 & 0 \\
\hline & b3 & -0.00000014 & 0.00058977 & 0 & 0 & 0 & 0 & 0 & 0 \\
\hline & b2 & 0.00000048 & -0.00030489 & 0 & 0 & 0 & 0 & 0 & 0 \\
\hline & b1 & 0.00000001 & 0.00004494 & -0.00001157 & 0.00007577 & -0.00000032 & 0.00000321 & 0.00000323 & -0.00002018 \\
\hline & b0 & 0 & 0.00000065 & 0.00000230 & 0.00000001 & -0.00000181 & -0.00000218 & -0.00000147 & 0.00000004 \\
\hline \multirow{6}{*}{$y_{3}$} & b5 & 0 & 0 & 0 & 0 & 0 & 0 & 0 & 0 \\
\hline & b4 & 0 & 0 & 0 & -0.00343149 & 0 & 0 & 0 & 0 \\
\hline & b3 & 0.00207776 & -0.00543070 & 0 & 0.00049288 & 0 & 0 & 0 & 0 \\
\hline & b2 & 0.00005860 & 0.00058305 & 0 & 0.00003009 & 0 & 0 & 0 & 0 \\
\hline & b1 & 0.00003830 & 0.00000057 & -0.00006850 & 0.00000019 & 0.00007767 & 0.00008932 & 0.00008184 & -0.00062480 \\
\hline & b0 & 0.00000054 & -0.00000007 & 0.00001848 & 0 & -0.00001248 & -0.00001503 & -0.00001028 & -0.00000336 \\
\hline \multirow{6}{*}{$y_{4}$} & b5 & 0 & 0 & 0 & 0 & 0 & 0 & 0 & 0 \\
\hline & b4 & 0 & 0 & 0 & 0 & 0 & 0 & 0 & 0 \\
\hline & b3 & 0 & -0.00132885 & 0 & 0 & 0 & 0 & 0 & 0 \\
\hline & b2 & 0.00268940 & -0.00018634 & 0 & 0 & 0 & 0 & 0 & 0 \\
\hline & b1 & 0.00014403 & 0.00006126 & -0.00013679 & 0.00068009 & 0.00007461 & 0.00008422 & 0.00007217 & -0.00021301 \\
\hline & b0 & 0.00001479 & -0.00000082 & 0.00001653 & 0.00001510 & -0.00000910 & -0.00001096 & -0.00000746 & -0.00000280 \\
\hline \multirow{6}{*}{$y_{5}$} & b5 & 0 & 0 & 0 & 0 & 0 & 0 & 0 & 0 \\
\hline & b4 & 0 & 0 & 0 & 0 & 0 & 0 & 0 & 0 \\
\hline & b3 & 0 & 0 & 0 & 0 & 0 & 0 & 0 & 0 \\
\hline & b2 & -0.00047125 & 0 & 0 & 0 & 0 & 0 & 0 & 0 \\
\hline & b1 & 0.00001740 & 0.00044177 & -0.00015009 & 0.00037935 & 0.00012115 & 0.00012678 & 0.00010285 & -0.00014484 \\
\hline & b0 & 0 & -0.00000577 & 0.00001293 & 0.00001324 & -0.00000646 & -0.00000780 & -0.00000531 & -0.00000265 \\
\hline \multirow{6}{*}{$y_{6}$} & b5 & -0.00571228 & 0 & 0 & 0 & 0 & 0 & 0 & 0 \\
\hline & b4 & -0.00032045 & 0 & 0 & 0 & 0 & 0 & 0 & 0 \\
\hline & b3 & 0.00002251 & 0.00052189 & 0 & 0 & 0 & 0 & 0 & 0 \\
\hline & b2 & 0.00000087 & -0.00031259 & 0 & 0 & 0 & 0 & 0 & 0 \\
\hline & b1 & 0.00000001 & 0.00004551 & -0.00010539 & 0.00004944 & 0.00008004 & 0.00009605 & 0.00007625 & 0.00001400 \\
\hline & b0 & 0 & -0.00000040 & 0.00001275 & 0.00004390 & -0.00000760 & -0.00000914 & -0.00000628 & -0.00003246 \\
\hline \multirow{6}{*}{$y_{7}$} & b5 & 0 & 0 & 0 & 0 & 0 & 0 & 0 & 0 \\
\hline & b4 & 0 & 0 & 0 & 0 & 0 & 0 & 0 & 0 \\
\hline & b3 & 0 & 0.00567759 & 0 & 0 & 0 & 0 & 0 & -0.00286620 \\
\hline & b2 & 0 & -0.00410884 & 0 & 0 & 0 & 0 & 0 & 0.06918149 \\
\hline & b1 & 0.00315703 & 0.00103824 & -0.26074940 & 0.03467888 & 0.02423986 & 0.02263684 & 0.02888788 & -0.00009883 \\
\hline & b0 & 0.00003633 & -0.00001601 & -0.00243858 & 0.00064345 & -0.00061709 & -0.00069309 & -0.00057488 & -0.00000185 \\
\hline \multirow{6}{*}{$y_{8}$} & b5 & 0 & 0 & 0 & 0 & 0 & 0 & 0 & 0 \\
\hline & b4 & 0 & 0 & 0 & 0 & 0 & 0 & 0 & 0 \\
\hline & b3 & -0.01982822 & 0 & 0 & 0 & 0 & 0 & 0 & 0 \\
\hline & b2 & 0.01031364 & 0 & 0 & 0 & 0 & 0 & 0 & 0 \\
\hline & b1 & 0.00000003 & 0.00773426 & -0.00014768 & -0.00014429 & 0.00832686 & 0.00965079 & 0.00631434 & -0.00292617 \\
\hline & b0 & 0.00000010 & -0.00015699 & 0.00013260 & 0.00000367 & -0.00087983 & -0.00105450 & -0.00065968 & -0.00001558 \\
\hline \multirow{6}{*}{$y_{9}$} & b5 & 0 & 0.00006324 & 0 & 0 & 0 & 0 & 0 & 0 \\
\hline & b4 & 0 & -0.00001981 & 0 & 0 & 0 & 0 & 0 & 0 \\
\hline & b3 & -0.00552608 & 0.00000592 & 0 & 0 & 0 & 0 & 0 & 0 \\
\hline & b2 & 0.00003670 & -0.00000004 & 0 & 0 & 0 & 0 & 0 & 0 \\
\hline & b1 & 0.00000400 & 0 & 0.00000103 & 0.00000114 & -0.00000837 & -0.00000779 & -0.00000414 & -0.00005138 \\
\hline & b0 & 0 & 0 & 0.00000005 & 0 & -0.00000131 & -0.00000163 & -0.00000094 & -0.00000037 \\
\hline \multirow{6}{*}{$y_{10}$} & b5 & 0 & 0 & 0 & 0 & 0 & 0 & 0 & 0 \\
\hline & b4 & 0 & 0 & 0 & 0 & 0 & 0 & 0 & 0 \\
\hline & b3 & 0 & 0 & 0 & 0 & 0 & 0 & 0 & 0 \\
\hline & b2 & 0 & 0 & 0 & 0 & 0 & 0 & 0 & 0 \\
\hline & b1 & 0.00396161 & 0.00276129 & 0.00005970 & 0.00000854 & 0.00987424 & 0.01017849 & 0.00922054 & -0.00024472 \\
\hline & b0 & 0.00002689 & -0.00006180 & -0.00000001 & -0.00000002 & -0.00071961 & -0.00084560 & -0.00054567 & -0.00000796 \\
\hline \multirow{6}{*}{$y_{11}$} & b5 & 0 & 0 & 0 & 0 & 0 & 0 & 0 & 0 \\
\hline & b4 & 0 & 0.00002798 & 0 & 0 & 0 & 0 & 0 & 0 \\
\hline & b3 & 0.00109664 & -0.00000455 & 0 & 0 & 0 & 0 & 0 & 0 \\
\hline & b2 & 0.00006766 & 0.00000192 & 0 & 0 & 0 & 0 & 0 & 0 \\
\hline & b1 & 0.00000707 & -0.00000005 & 0.00000012 & 0.00000106 & 0.00003534 & 0.00002000 & 0.00001609 & -0.00001741 \\
\hline & b0 & 0.00000023 & 0 & 0.00000007 & 0.00000003 & -0.00000207 & -0.00000248 & -0.00000152 & 0.00000005 \\
\hline
\end{tabular}


Table 22 - Plant 2 - Transfer Function Denominator Parameters

\begin{tabular}{|c|c|c|c|c|c|c|c|c|c|}
\hline P2 & D & $u_{l}$ & $u_{2}$ & $u_{3}$ & $u_{4}$ & $u_{5}$ & $u_{6}$ & $u_{7}$ & $d_{l}$ \\
\hline \multirow{6}{*}{$y_{1}$} & a5 & 1 & 1 & 0 & 0 & 0 & 0 & 0 & 0 \\
\hline & a4 & 0.01714772 & 0.11294159 & 0 & 0 & 0 & 0 & 0 & 0 \\
\hline & a3 & 0.00005084 & 0.01412991 & 1 & 0 & 0 & 0 & 0 & 0 \\
\hline & $\mathrm{a} 2$ & 0.00000050 & 0.00006624 & 0.10680783 & 1 & 1 & 1 & 1 & 1 \\
\hline & a1 & 0 & 0.00000004 & 0.00130945 & 0.01303862 & 0.01284120 & 0.01292737 & 0.01302207 & 0.00251888 \\
\hline & $\mathrm{a} 0$ & 0 & 0 & 0.00000193 & 0.00001958 & 0.00001892 & 0.00001897 & 0.00001944 & 0.00000282 \\
\hline \multirow{6}{*}{$y_{2}$} & a5 & 1 & 0 & 0 & 0 & 0 & 0 & 0 & 0 \\
\hline & $\mathrm{a} 4$ & 0.12412928 & 0 & 0 & 0 & 0 & 0 & 0 & 0 \\
\hline & a3 & 0.00391192 & 1 & 0 & 0 & 0 & 0 & 0 & 0 \\
\hline & a2 & 0.00004761 & 0.14063439 & 1 & 1 & 1 & 1 & 1 & 1 \\
\hline & a1 & 0.00000007 & 0.00253530 & 0.04425476 & 0.00183187 & 0.01788072 & 0.01741509 & 0.01830251 & 0 \\
\hline & $\mathrm{a} 0$ & 0 & 0.00000411 & 0.00007389 & 0.00000013 & 0.00002620 & 0.00002571 & 0.00002731 & 0.00000026 \\
\hline \multirow{6}{*}{$y_{3}$} & a5 & 0 & 0 & 0 & 0 & 0 & 0 & 0 & 0 \\
\hline & a4 & 0 & 0 & 0 & 1 & 0 & 0 & 0 & 0 \\
\hline & a3 & 1 & 1 & 0 & 0.04797087 & 0 & 0 & 0 & 0 \\
\hline & $\mathrm{a} 2$ & 0.06203264 & 0.03188063 & 1 & 0.00033828 & 1 & 1 & 1 & 1 \\
\hline & a1 & 0.00062186 & 0.00027210 & 0.02741094 & 0.00000053 & 0.02559482 & 0.02515364 & 0.02624496 & 0.01041877 \\
\hline & $\mathrm{a} 0$ & 0.00000089 & 0.00000037 & 0.00004411 & 0 & 0.00003960 & 0.00003883 & 0.00004113 & 0.00000349 \\
\hline \multirow{6}{*}{$y_{4}$} & a5 & 0 & 0 & 0 & 0 & 0 & 0 & 0 & 0 \\
\hline & a4 & 0 & 0 & 0 & 0 & 0 & 0 & 0 & 0 \\
\hline & a3 & 0 & 1 & 0 & 0 & 0 & 0 & 0 & 0 \\
\hline & $\mathrm{a} 2$ & 1 & 0.12310097 & 1 & 1 & 1 & 1 & 1 & 1 \\
\hline & a1 & 0.01341609 & 0.00159427 & 0.01573947 & 0.01439703 & 0.01481716 & 0.01466346 & 0.01500543 & 0.00671748 \\
\hline & $\mathrm{a} 0$ & 0.00001956 & 0.00000240 & 0.00002407 & 0.00002200 & 0.00002201 & 0.00002171 & 0.00002255 & 0.00000885 \\
\hline \multirow{6}{*}{$y_{5}$} & a5 & 0 & 0 & 0 & 0 & 0 & 0 & 0 & 0 \\
\hline & a4 & 0 & 0 & 0 & 0 & 0 & 0 & 0 & 0 \\
\hline & a3 & 1 & 0 & 0 & 0 & 0 & 0 & 0 & 0 \\
\hline & a2 & 0.01705386 & 1 & 1 & 1 & 1 & 1 & 1 & 1 \\
\hline & a1 & 0.00002035 & 0.00915569 & 0.01163580 & 0.01160280 & 0.01099575 & 0.01086539 & 0.01111255 & 0.00636816 \\
\hline & $\mathrm{a} 0$ & 0 & 0.00001440 & 0.00001724 & 0.00001732 & 0.00001618 & 0.00001582 & 0.00001648 & 0.00000831 \\
\hline \multirow{6}{*}{$y_{6}$} & a5 & 1 & 0 & 0 & 0 & 0 & 0 & 0 & 0 \\
\hline & $\mathrm{a} 4$ & 0.11480197 & 0 & 0 & 0 & 0 & 0 & 0 & 0 \\
\hline & a3 & 0.00286161 & 1 & 0 & 0 & 0 & 0 & 0 & 0 \\
\hline & $\mathrm{a} 2$ & 0.00002125 & 0.12008429 & 1 & 1 & 1 & 1 & 1 & 1 \\
\hline & a1 & 0.00000003 & 0.00425409 & 0.06397040 & 0.21872143 & 0.04051925 & 0.03956747 & 0.04214022 & 0.25722627 \\
\hline & $\mathrm{a} 0$ & 0 & 0.00000714 & 0.00011094 & 0.00038591 & 0.00006576 & 0.00006407 & 0.00006928 & 0.00026217 \\
\hline \multirow{6}{*}{$y_{7}$} & a5 & 0 & 0 & 0 & 0 & 0 & 0 & 0 & 0 \\
\hline & $\mathrm{a} 4$ & 0 & 0 & 0 & 0 & 0 & 0 & 0 & 0 \\
\hline & a3 & 0 & 1 & 0 & 0 & 0 & 0 & 0 & 1 \\
\hline & $\mathrm{a} 2$ & 1 & 0.10828070 & 1 & 1 & 1 & 1 & 1 & 1.42914642 \\
\hline & a1 & 0.03846274 & 0.01387072 & 0.76201213 & 0.38478220 & 0.62306946 & 0.53563021 & 0.79758883 & 0.03383366 \\
\hline & $\mathrm{a} 0$ & 0.00025870 & 0.00020105 & 0.01085486 & 0.00452169 & 0.01114221 & 0.00949230 & 0.01438864 & 0.00008011 \\
\hline \multirow{6}{*}{$y_{8}$} & a5 & 0 & 0 & 0 & 0 & 0 & 0 & 0 & 0 \\
\hline & a4 & 0 & 0 & 0 & 0 & 0 & 0 & 0 & 0 \\
\hline & a3 & 1 & 0 & 0 & 0 & 0 & 0 & 0 & 0 \\
\hline & $\mathrm{a} 2$ & 0.01125410 & 1 & 1 & 1 & 1 & 1 & 1 & 1 \\
\hline & a1 & 0.00000920 & 0.06213124 & 0.53034874 & 0.04302060 & 0.21497917 & 0.21378488 & 0.21402466 & 0.02060087 \\
\hline & $\mathrm{a} 0$ & 0.00000010 & 0.00063978 & 0.00506432 & 0.00034658 & 0.00225555 & 0.00221773 & 0.00225956 & 0.00005659 \\
\hline \multirow{6}{*}{$y_{9}$} & a5 & 0 & 1 & 0 & 0 & 0 & 0 & 0 & 0 \\
\hline & a4 & 0 & 0.14695206 & 0 & 0 & 0 & 0 & 0 & 0 \\
\hline & a3 & 1 & 0.00245709 & 0 & 0 & 0 & 0 & 0 & 0 \\
\hline & $a 2$ & 0.00941951 & 0.00001660 & 1 & 1 & 1 & 1 & 1 & 1 \\
\hline & a1 & 0.00000759 & 0.00000008 & 0.01011649 & 0 & 0.00924142 & 0.00907954 & 0.00885333 & 0.00381538 \\
\hline & $\mathrm{a} 0$ & 0 & 0 & 0.00001189 & 0.00000028 & 0.00001081 & 0.00001079 & 0.00001083 & 0.00000434 \\
\hline \multirow{6}{*}{$y_{10}$} & a5 & 0 & 0 & 0 & 0 & 0 & 0 & 0 & 0 \\
\hline & a4 & 0 & 0 & 0 & 0 & 0 & 0 & 0 & 0 \\
\hline & a3 & 0 & 0 & 0 & 0 & 0 & 0 & 0 & 0 \\
\hline & $\mathrm{a} 2$ & 1 & 1 & 1 & 1 & 1 & 1 & 1 & 1 \\
\hline & a1 & 0.02159305 & 0.09591554 & 0.01254107 & 0.00100962 & 0.71853627 & 0.66029395 & 0.74081993 & 0.02447674 \\
\hline & $\mathrm{a} 0$ & 0.00008602 & 0.00120310 & 0 & 0 & 0.00755120 & 0.00696994 & 0.00783462 & 0.00011159 \\
\hline \multirow{6}{*}{$y_{11}$} & a5 & 0 & 1 & 0 & 0 & 0 & 0 & 0 & 0 \\
\hline & $\mathrm{a} 4$ & 0 & 0.39354906 & 0 & 0 & 0 & 0 & 0 & 0 \\
\hline & a3 & 1 & 0.04280596 & 0 & 0 & 0 & 0 & 0 & 0 \\
\hline & $\mathrm{a} 2$ & 0.06007570 & 0.00107141 & 1 & 1 & 1 & 1 & 1 & 1 \\
\hline & a1 & 0.00077089 & 0.00000320 & 0.01523808 & 0.01041895 & 0.01915250 & 0.01859453 & 0.01913125 & 0 \\
\hline & $\mathrm{a} 0$ & 0.00000113 & 0 & 0.00002304 & 0.00001148 & 0.00003061 & 0.00002825 & 0.00002939 & 0.00000028 \\
\hline
\end{tabular}


Table 23 - Plant 3 - Transfer Function Numerator Parameters

\begin{tabular}{|c|c|c|c|c|c|c|c|c|c|}
\hline P3 & $\mathrm{N}$ & $u_{1}$ & $u_{2}$ & $u_{3}$ & $u_{4}$ & $u_{5}$ & $u_{6}$ & $u_{7}$ & $d_{1}$ \\
\hline \multirow{6}{*}{$y_{1}$} & b5 & -0.00161290 & 0.00008043 & 0 & 0 & 0 & 0 & 0 & 0 \\
\hline & b4 & -0.00001942 & 0.00000408 & 0 & 0 & 0 & 0 & 0 & 0 \\
\hline & b3 & 0.00039506 & -0.00012489 & -0.00054449 & 0 & 0 & 0 & 0 & 0 \\
\hline & b2 & 0.00002450 & 0.00007704 & -0.00884968 & 0 & 0 & 0 & 0 & 0 \\
\hline & b1 & 0.00000033 & 0.00000079 & -0.00001294 & 0.02024509 & 0.00112454 & 0.00153559 & 0.00096894 & -0.00026309 \\
\hline & b0 & 0 & 0 & 0.00000782 & 0.00025084 & -0.00013057 & -0.00016574 & -0.00011712 & -0.00000148 \\
\hline \multirow{6}{*}{$y_{2}$} & b5 & -0.00218441 & 0 & 0 & 0 & 0 & 0 & 0 & 0 \\
\hline & b4 & 0.01040511 & 0 & 0 & 0 & 0 & 0 & 0 & 0 \\
\hline & b3 & -0.00799950 & 0.01532212 & 0 & 0 & 0 & 0 & 0 & 0 \\
\hline & b2 & 0.00252486 & -0.01151497 & 0 & 0 & 0 & 0 & 0 & 0 \\
\hline & b1 & 0.00013528 & 0.00299862 & 0.00201796 & 0.03148049 & -0.00191849 & -0.00234263 & -0.00156138 & -0.00157588 \\
\hline & b0 & 0.00000094 & 0.00001234 & 0.00000927 & 0.00033056 & -0.00000605 & -0.00000564 & -0.00000660 & -0.00000764 \\
\hline \multirow{6}{*}{$y_{3}$} & b5 & 0 & 0 & 0 & 0 & 0 & 0 & 0 & 0 \\
\hline & b4 & 0 & 0 & 0 & 0.00019890 & 0 & 0 & 0 & 0 \\
\hline & b3 & 0.01095129 & 0.02010699 & 0 & 4.58116571 & 0 & 0 & 0 & 0 \\
\hline & b2 & -0.02483896 & -0.01335635 & 0 & 2.98617164 & 0 & 0 & 0 & 0 \\
\hline & b1 & 0.00820037 & 0.00363976 & -0.09200937 & 0.44223885 & 0.02100254 & 0.02610078 & 0.01658650 & -0.00424600 \\
\hline & b0 & 0.00009258 & -0.00004286 & 0.01091960 & 0.00440818 & -0.00213627 & -0.00259634 & -0.00170705 & -0.00001550 \\
\hline \multirow{6}{*}{$y_{4}$} & b5 & 0 & 0 & 0 & 0 & 0 & 0 & 0 & 0 \\
\hline & b4 & 0.00682135 & 0 & 0 & 0 & 0 & 0 & 0 & 0 \\
\hline & b3 & -0.00816945 & 0.01036191 & 0 & 0 & 0 & 0 & 0 & 0 \\
\hline & b2 & 0.00188302 & -0.00820750 & 0 & 0 & 0 & 0 & 0 & 0 \\
\hline & b1 & 0.00021007 & 0.00225827 & -0.11009818 & 0.14720726 & 0.01300538 & 0.01604061 & 0.01049799 & -0.00349863 \\
\hline & b0 & 0.00000160 & -0.00003513 & 0.00871862 & 0.00214211 & -0.00164513 & -0.00194120 & -0.00133927 & -0.00002119 \\
\hline \multirow{6}{*}{$y_{5}$} & b5 & 0 & 0 & 0 & 0 & 0 & 0 & 0 & 0 \\
\hline & b4 & 0 & 0 & 0 & 0 & 0 & 0 & 0 & 0 \\
\hline & b3 & 0 & 0 & 0 & 0 & 0 & 0 & 0 & 0 \\
\hline & b2 & -0.00192037 & 0 & 0 & 0 & 0 & 0 & 0 & 0 \\
\hline & b1 & 0.00018122 & 0.00856747 & -0.03127132 & 0.11498499 & 0.00505431 & 0.00604381 & 0.00407196 & -0.01213690 \\
\hline & b0 & 0.00015405 & -0.00014082 & 0.00214370 & 0.00263465 & -0.00050539 & -0.00058055 & -0.00041487 & -0.01756267 \\
\hline \multirow{6}{*}{$y_{6}$} & b5 & -0.00075758 & 0 & 0 & 0 & 0 & 0 & 0 & 0 \\
\hline & b4 & 0.00575897 & 0 & 0 & 0 & 0 & 0 & 0 & 0 \\
\hline & b3 & -0.00520181 & 0.01155428 & 0 & 0 & 0 & 0 & 0 & 0 \\
\hline & b2 & 0.00169798 & -0.00744122 & 0 & 0 & 0 & 0 & 0 & 0 \\
\hline & b1 & 0.00009839 & 0.00187977 & -0.03313173 & 0.11388954 & 0.00958047 & 0.01191169 & 0.00748200 & -0.00189899 \\
\hline & b0 & 0.00000047 & -0.00001988 & 0.00321106 & 0.01219921 & -0.00088504 & -0.00107870 & -0.00070246 & -0.00000632 \\
\hline \multirow{6}{*}{$y_{7}$} & b5 & 0 & 0 & 0 & 0 & 0 & 0 & 0 & 0 \\
\hline & b4 & 0 & 0 & 0 & 0 & 0 & 0 & 0 & 0 \\
\hline & b3 & 0 & 0.00121229 & 0 & 0 & 0 & 0 & 0 & 0 \\
\hline & b2 & 0 & -0.00178098 & 0 & 0 & 0 & 0 & 0 & 0 \\
\hline & b1 & 0.00267801 & 0.00049209 & -0.25565186 & 0.03173403 & 0.03292907 & 0.02799681 & 0.04160051 & 9.39293016 \\
\hline & b0 & 0.00002573 & -0.00000964 & -0.00193167 & 0.00041422 & -0.00074972 & -0.00078082 & -0.00072543 & -0.05676959 \\
\hline \multirow{6}{*}{$y_{8}$} & b5 & 0 & 0 & 0 & 0 & 0 & 0 & 0 & 0 \\
\hline & b4 & 0 & 0 & 0 & 0 & 0 & 0 & 0 & 0 \\
\hline & b3 & 0.09205938 & 0 & 0 & 0 & 0 & 0 & 0 & 0 \\
\hline & b2 & 0.00620517 & 0 & 0 & 0 & 0 & 0 & 0 & 0 \\
\hline & b1 & 0.00003672 & 0.00437241 & -0.00013281 & 0.00005851 & 0.00585489 & 0.00675517 & 0.00442002 & -0.00165014 \\
\hline & b0 & 0.00000002 & -0.00012916 & 0.00005328 & -0.00000002 & -0.00062297 & -0.00075644 & -0.00046743 & -0.00000489 \\
\hline \multirow{6}{*}{$y_{9}$} & b5 & 0 & -0.00059059 & 0 & 0 & 0 & 0 & 0 & 0 \\
\hline & b4 & 0 & 0.00041521 & 0 & 0 & 0 & 0 & 0 & 0 \\
\hline & b3 & -0.00598629 & -0.00010556 & 0 & 0 & 0 & 0 & 0 & 0 \\
\hline & b2 & 0.00318122 & 0.00004742 & 0 & 0 & 0 & 0 & 0 & 0 \\
\hline & b1 & 0.00036420 & -0.00000031 & 0.00005921 & -0.00001364 & -0.00059423 & -0.00076368 & -0.00045953 & -0.00069813 \\
\hline & b0 & 0.00000240 & 0 & 0.00000100 & 0.00000055 & -0.00000009 & -0.00000020 & -0.00000115 & -0.00000529 \\
\hline \multirow{6}{*}{$y_{10}$} & b5 & 0 & 0 & 0 & 0 & 0 & 0 & 0 & 0 \\
\hline & b4 & 0 & 0 & 0 & 0 & 0 & 0 & 0 & 0 \\
\hline & b3 & 0 & 0 & 0 & 0 & 0 & 0 & 0 & 0 \\
\hline & b2 & 0 & 0 & 0 & 0 & 0 & 0 & 0 & 0 \\
\hline & b1 & 0.00310277 & 0.00173269 & 0.00004467 & 0.00000963 & 0.00963113 & 0.00705205 & 0.00904038 & -0.00051427 \\
\hline & b0 & 0.00001797 & -0.00006038 & -0.00000001 & 0.00000041 & -0.00066352 & -0.00058644 & -0.00048999 & -0.00000358 \\
\hline \multirow{6}{*}{$y_{11}$} & b5 & 0 & 0 & 0 & 0 & 0 & 0 & 0 & 0 \\
\hline & b4 & 0 & 0.00007460 & 0 & 0 & 0 & 0 & 0 & 0 \\
\hline & b3 & -0.00037588 & 0.00003362 & 0 & 0 & 0 & 0 & 0 & 0 \\
\hline & b2 & 0.00267446 & 0.00003877 & 0 & 0 & 0 & 0 & 0 & 0 \\
\hline & b1 & 0.00050490 & -0.00000037 & -0.00002233 & -0.00004926 & 0.00224305 & 0.00415768 & 0.00181170 & -0.00058252 \\
\hline & b0 & 0.00000265 & -0.00000009 & 0.00001965 & 0.00000297 & -0.00030195 & -0.00051715 & -0.00023705 & -0.00000194 \\
\hline
\end{tabular}


Table 24 - Plant 3 - Transfer Function Denominator Parameters

\begin{tabular}{|c|c|c|c|c|c|c|c|c|c|}
\hline P3 & $\mathrm{D}$ & $u_{1}$ & $u_{2}$ & $u_{3}$ & $u_{4}$ & $u_{5}$ & $u_{6}$ & $u_{7}$ & $d_{1}$ \\
\hline \multirow{6}{*}{$y_{1}$} & a5 & 1 & 1 & 0 & 0 & 0 & 0 & 0 & 0 \\
\hline & a4 & 0.60616366 & 0.26006504 & 0 & 0 & 0 & 0 & 0 & 0 \\
\hline & a3 & 0.04003953 & 0.04040114 & 1 & 0 & 0 & 0 & 0 & 0 \\
\hline & a2 & 0.00083667 & 0.00285457 & 0.67672652 & 1 & 1 & 1 & 1 & 1 \\
\hline & a1 & 0.00000422 & 0.00004973 & 0.02802616 & 0.34553608 & 0.39651350 & 0.41650526 & 0.43808977 & 0.01669236 \\
\hline & $\mathrm{a} 0$ & 0 & 0.00000023 & 0.00019015 & 0.00290484 & 0.00327246 & 0.00341690 & 0.00367504 & 0.00004821 \\
\hline \multirow{6}{*}{$y_{2}$} & a5 & 1 & 0 & 0 & 0 & 0 & 0 & 0 & 0 \\
\hline & a4 & 0.73338773 & 0 & 0 & 0 & 0 & 0 & 0 & 0 \\
\hline & a3 & 0.30252888 & 1 & 0 & 0 & 0 & 0 & 0 & 0 \\
\hline & $\mathrm{a} 2$ & 0.03196168 & 0.13151790 & 1 & 1 & 1 & 1 & 1 & 1 \\
\hline & a1 & 0.00055550 & 0.01411709 & 0.02875371 & 0.37612675 & 0.01499550 & 0.01383684 & 0.01664834 & 0.02144646 \\
\hline & $\mathrm{a} 0$ & 0.00000227 & 0.00014080 & 0.00010432 & 0.00342748 & 0.00003450 & 0.00002557 & 0.00004824 & 0.00005771 \\
\hline \multirow{6}{*}{$y_{3}$} & a5 & 0 & 0 & 0 & 0 & 0 & 0 & 0 & 0 \\
\hline & a4 & 0 & 0 & 0 & 1 & 0 & 0 & 0 & 0 \\
\hline & a3 & 1 & 1 & 0 & 30.57056552 & 0 & 0 & 0 & 0 \\
\hline & $\mathrm{a} 2$ & 0.48323275 & 0.12222426 & 1 & 12.99983459 & 1 & 1 & 1 & 1 \\
\hline & a1 & 0.02350459 & 0.01333325 & 1.15251139 & 1.24281174 & 0.34386181 & 0.34402516 & 0.33617157 & 0.02582544 \\
\hline & $\mathrm{a} 0$ & 0.00015477 & 0.00024303 & 0.02663119 & 0.01061586 & 0.00711138 & 0.00704405 & 0.00710418 & 0.00006556 \\
\hline \multirow{6}{*}{$y_{4}$} & a5 & 0 & 0 & 0 & 0 & 0 & 0 & 0 & 0 \\
\hline & a4 & 1 & 0 & 0 & 0 & 0 & 0 & 0 & 0 \\
\hline & a3 & 0.24682552 & 1 & 0 & 0 & 0 & 0 & 0 & 0 \\
\hline & a2 & 0.02911368 & 0.10920344 & 1 & 1 & 1 & 1 & 1 & 1 \\
\hline & a1 & 0.00052769 & 0.01078674 & 1.16076062 & 0.35068568 & 0.40412591 & 0.39464666 & 0.40185801 & 0.02103288 \\
\hline & $\mathrm{a} 0$ & 0.00000218 & 0.00010262 & 0.01295549 & 0.00313854 & 0.00409003 & 0.00396640 & 0.00413259 & 0.00006688 \\
\hline \multirow{6}{*}{$y_{5}$} & a5 & 0 & 0 & 0 & 0 & 0 & 0 & 0 & 0 \\
\hline & $\mathrm{a} 4$ & 0 & 0 & 0 & 0 & 0 & 0 & 0 & 0 \\
\hline & a3 & 1 & 0 & 0 & 0 & 0 & 0 & 0 & 0 \\
\hline & $\mathrm{a} 2$ & 0.19728464 & 1 & 1 & 1 & 1 & 1 & 1 & 1 \\
\hline & a1 & 0.03373426 & 0.04940690 & 0.37952403 & 0.46431275 & 0.17888196 & 0.17098216 & 0.17959950 & 9.65369794 \\
\hline & $\mathrm{a} 0$ & 0.00021699 & 0.00033173 & 0.00289534 & 0.00343470 & 0.00123983 & 0.00117319 & 0.00126066 & 0.05530446 \\
\hline \multirow{6}{*}{$y_{6}$} & a5 & 1 & 0 & 0 & 0 & 0 & 0 & 0 & 0 \\
\hline & $\mathrm{a} 4$ & 0.65957664 & 0 & 0 & 0 & 0 & 0 & 0 & 0 \\
\hline & a3 & 0.22269097 & 1 & 0 & 0 & 0 & 0 & 0 & 0 \\
\hline & $\mathrm{a} 2$ & 0.02250019 & 0.13949382 & 1 & 1 & 1 & 1 & 1 & 1 \\
\hline & a1 & 0.00051595 & 0.01467835 & 0.86032882 & 1.07844538 & 0.28219311 & 0.28348602 & 0.27530588 & 0.02936023 \\
\hline & $\mathrm{a} 0$ & 0.00000196 & 0.00033151 & 0.02757344 & 0.06770926 & 0.00773520 & 0.00766312 & 0.00773408 & 0.00007096 \\
\hline \multirow{6}{*}{$y_{7}$} & a5 & 0 & 0 & 0 & 0 & 0 & 0 & 0 & 0 \\
\hline & a4 & 0 & 0 & 0 & 0 & 0 & 0 & 0 & 0 \\
\hline & a3 & 0 & 1 & 0 & 0 & 0 & 0 & 0 & 0 \\
\hline & $\mathrm{a} 2$ & 1 & 0.08725273 & 1 & 1 & 1 & 1 & 1 & 1 \\
\hline & a1 & 0.03312328 & 0.01017913 & 0.73885014 & 0.33524447 & 0.94763699 & 0.75232933 & 1.26428402 & 276.11678502 \\
\hline & $\mathrm{a} 0$ & 0.00018177 & 0.00012047 & 0.00858640 & 0.00288218 & 0.01360218 & 0.01074938 & 0.01827899 & 2.39957106 \\
\hline \multirow{6}{*}{$y_{8}$} & a5 & 0 & 0 & 0 & 0 & 0 & 0 & 0 & 0 \\
\hline & $\mathrm{a} 4$ & 0 & 0 & 0 & 0 & 0 & 0 & 0 & 0 \\
\hline & a3 & 1 & 0 & 0 & 0 & 0 & 0 & 0 & 0 \\
\hline & a2 & 0.01057821 & 1 & 1 & 1 & 1 & 1 & 1 & 1 \\
\hline & a1 & 0.00004423 & 0.06299703 & 0.25806181 & 0.00496132 & 0.19510464 & 0.19640850 & 0.19460712 & 0.01029938 \\
\hline & $\mathrm{a} 0$ & 0.00000001 & 0.00052878 & 0.00205527 & 0 & 0.00163304 & 0.00162326 & 0.00163627 & 0.00001868 \\
\hline \multirow{6}{*}{$y_{9}$} & a5 & 0 & 1 & 0 & 0 & 0 & 0 & 0 & 0 \\
\hline & $\mathrm{a} 4$ & 0 & 0.26640812 & 0 & 0 & 0 & 0 & 0 & 0 \\
\hline & a3 & 1 & 0.04281507 & 0 & 0 & 0 & 0 & 0 & 0 \\
\hline & a2 & 0.17198260 & 0.00341701 & 1 & 1 & 1 & 1 & 1 & 1 \\
\hline & a1 & 0.00250734 & 0.00006023 & 0.05626396 & 0.07767206 & 0.00599493 & 0.00616802 & 0.00885345 & 0.02489282 \\
\hline & $\mathrm{a} 0$ & 0.00000701 & 0.00000019 & 0.00025698 & 0.00041129 & 0 & 0.00000066 & 0.00001368 & 0.00006095 \\
\hline \multirow{6}{*}{$y_{10}$} & a5 & 0 & 0 & 0 & 0 & 0 & 0 & 0 & 0 \\
\hline & $\mathrm{a} 4$ & 0 & 0 & 0 & 0 & 0 & 0 & 0 & 0 \\
\hline & a3 & 0 & 0 & 0 & 0 & 0 & 0 & 0 & 0 \\
\hline & a2 & 1 & 1 & 1 & 1 & 1 & 1 & 1 & 1 \\
\hline & a1 & 0.01741610 & 0.10980178 & 0.00917391 & 0.02118988 & 0.83101837 & 0.55217599 & 0.83145014 & 0.01907029 \\
\hline & $\mathrm{a} 0$ & 0.00005770 & 0.00117154 & 0 & 0.00013578 & 0.00700103 & 0.00471769 & 0.00707324 & 0.00004898 \\
\hline \multirow{6}{*}{$y_{11}$} & a5 & 0 & 1 & 0 & 0 & 0 & 0 & 0 & 0 \\
\hline & a4 & 0 & 0.28079827 & 0 & 0 & 0 & 0 & 0 & 0 \\
\hline & a3 & 1 & 0.04833173 & 0 & 0 & 0 & 0 & 0 & 0 \\
\hline & $\mathrm{a} 2$ & 0.19768300 & 0.00445632 & 1 & 1 & 1 & 1 & 1 & 1 \\
\hline & a1 & 0.00353637 & 0.00019380 & 0.54226850 & 0.13936678 & 0.33816196 & 0.46152256 & 0.34547457 & 0.01687142 \\
\hline & $\mathrm{a} 0$ & 0.00001309 & 0.00000247 & 0.00646498 & 0.00174467 & 0.00440564 & 0.00595548 & 0.00453658 & 0.00003605 \\
\hline
\end{tabular}


Table 25 - Plant 4 - Transfer Function Numerator Parameters

\begin{tabular}{|c|c|c|c|c|c|c|c|c|c|}
\hline $\mathrm{P} 4$ & $\mathrm{~N}$ & $u_{1}$ & $u_{2}$ & $u_{3}$ & $u_{4}$ & $u_{5}$ & $u_{6}$ & $u_{7}$ & $d_{1}$ \\
\hline \multirow{6}{*}{$y_{1}$} & b5 & 0.00043288 & -0.00042324 & 0 & 0 & 0 & 0 & 0 & 0 \\
\hline & b4 & -0.00012997 & -0.00014724 & 0 & 0 & 0 & 0 & 0 & 0 \\
\hline & b3 & -0.00005906 & 0.00026506 & -0.00038292 & 0 & 0 & 0 & 0 & 0 \\
\hline & b2 & 0.00001985 & -0.00000808 & -0.01085542 & 0 & 0 & 0 & 0 & 0 \\
\hline & b1 & 0.00000636 & -0.00000001 & 0.00022437 & 0.02584718 & 0.00039002 & 0.00060525 & 0.00019113 & 0.00001456 \\
\hline & b0 & 0.00000014 & 0 & 0.00000524 & 0.00050729 & -0.00004249 & -0.00006348 & -0.00002933 & -0.00001560 \\
\hline \multirow{6}{*}{$y_{2}$} & b5 & 0.00748264 & 0 & 0 & 0 & 0 & 0 & 0 & 0 \\
\hline & b4 & -0.00732130 & 0 & 0 & 0 & 0 & 0 & 0 & 0 \\
\hline & b3 & 0.00149776 & -0.15270749 & 0 & 0 & 0 & 0 & 0 & 0 \\
\hline & b2 & 0.00045168 & 0.03307379 & 0 & 0 & 0 & 0 & 0 & 0 \\
\hline & b1 & 0.00006083 & 0.00011826 & 0.00307673 & 0.05870695 & 0.00739350 & 0.01995306 & 0.00341213 & 0.00109194 \\
\hline & b0 & 0.00000106 & -0.00003475 & 0.00003781 & 0.00145047 & -0.00062422 & -0.00151898 & -0.00036390 & -0.00016813 \\
\hline \multirow{6}{*}{$y_{3}$} & b5 & 0 & 0 & 0 & 0 & 0 & 0 & 0 & 0 \\
\hline & b4 & 0 & 0 & 0 & 0.04816343 & 0 & 0 & 0 & 0.08076511 \\
\hline & b3 & -0.06485669 & -0.32827011 & 0 & 0.19584792 & 0 & 0 & 0 & -0.00619779 \\
\hline & b2 & 0.00937300 & 0.06612935 & 0 & 0.00986393 & 0 & 0 & 0 & 0.00016669 \\
\hline & b1 & 0.00381077 & -0.00016854 & -0.12703380 & 0.00076320 & 0.03543603 & 0.05761104 & 0.028853866 & -0.00002911 \\
\hline & b0 & 0.00003582 & -0.00007007 & 0.02879057 & 0.00000912 & -0.00354053 & -0.00578245 & -0.00335789 & -0.00000026 \\
\hline \multirow{6}{*}{$y_{4}$} & b5 & 0 & 0 & 0 & 0 & 0 & 0 & 0 & 0 \\
\hline & b4 & -0.01084749 & 0 & 0 & 0 & 0 & 0 & 0 & 0 \\
\hline & b3 & -0.00401664 & -0.16106886 & 0 & 0 & 0 & 0 & 0 & 0 \\
\hline & b2 & 0.00261844 & 0.02277745 & 0 & 0 & 0 & 0 & 0 & 0 \\
\hline & b1 & 0.00005947 & 0.00144328 & -0.16795744 & 0.27042419 & 0.00991176 & 0.01836889 & 0.00481536 & 0.00309991 \\
\hline & b0 & 0.00000018 & -0.00007981 & 0.02239373 & 0.00698732 & -0.00115866 & -0.00199521 & -0.00079413 & -0.00048102 \\
\hline \multirow{6}{*}{$y_{5}$} & b5 & 0 & 0 & 0 & 0 & 0 & 0 & 0 & 0 \\
\hline & b4 & 0 & 0 & 0 & 0 & 0 & 0 & 0 & 0 \\
\hline & b3 & 0 & 0 & 0 & 0 & 0 & 0 & 0 & 0 \\
\hline & b2 & -0.00636195 & 0 & 0 & 0 & 0 & 0 & 0 & 0 \\
\hline & b1 & 0.00175894 & 0.05291509 & -0.07136034 & 0.32368719 & 0.00342056 & 0.00736602 & 0.00163129 & 0.00260190 \\
\hline & b0 & 0.00004425 & -0.00164852 & 0.00816364 & 0.01561208 & -0.00063047 & -0.00090142 & -0.00045527 & -0.00029303 \\
\hline \multirow{6}{*}{$y_{6}$} & b5 & 0.00882574 & 0 & 0 & 0 & 0 & 0 & 0 & 0 \\
\hline & b4 & -0.00787106 & 0 & 0 & 0 & 0 & 0 & 0 & -0.04571486 \\
\hline & b3 & 0.00089650 & -0.15479664 & 0 & 0 & 0 & 0 & 0 & -0.00599902 \\
\hline & b2 & 0.00075197 & 0.03394245 & 0 & 0 & 0 & 0 & 0 & -0.00088396 \\
\hline & b1 & 0.00006102 & -0.00006556 & 0.00507948 & 0.14924099 & 0.01770524 & 0.02990788 & 0.01561167 & -0.00003584 \\
\hline & b0 & 0.00000050 & -0.00003599 & 0.00070214 & 0.00585941 & -0.00169702 & -0.00283140 & -0.00172972 & 0 \\
\hline \multirow{6}{*}{$y_{7}$} & b5 & 0 & 0 & 0 & 0 & 0 & 0 & 0 & 0 \\
\hline & b4 & 0 & 0 & 0 & 0 & 0 & 0 & 0 & 0 \\
\hline & b3 & 0 & -0.05924095 & 0 & 0 & 0 & 0 & 0 & 0 \\
\hline & b2 & 0 & 0.01000714 & 0 & 0 & 0 & 0 & 0 & 0 \\
\hline & b1 & 0.00765392 & 0.00032933 & -0.29734723 & 0.065888804 & 0.01448028 & 0.01965943 & 0.01441531 & 0.02723419 \\
\hline & b0 & 0.00005839 & -0.00002389 & -0.00325086 & 0.00177505 & -0.00077638 & -0.00118539 & -0.00071736 & -0.00061958 \\
\hline \multirow{6}{*}{$y_{8}$} & b5 & 0 & 0 & 0 & 0 & 0 & 0 & 0 & 0 \\
\hline & b4 & 0 & 0 & 0 & 0 & 0 & 0 & 0 & 0 \\
\hline & b3 & -0.00909860 & 0 & 0 & 0 & 0 & 0 & 0 & 0 \\
\hline & b2 & 0.00234450 & 0 & 0 & 0 & 0 & 0 & 0 & 0 \\
\hline & b1 & 0.00292877 & 0.07580051 & 0.00115489 & -0.00218057 & 0.01005627 & 0.01786067 & 0.00945423 & 0.00490081 \\
\hline & b0 & 0.00006589 & -0.00255974 & -0.00002838 & 0.00008663 & -0.00064841 & -0.00119367 & -0.00056697 & -0.00030644 \\
\hline \multirow{6}{*}{$y_{9}$} & b5 & 0 & 0.03765047 & 0 & 0 & 0 & 0 & 0 & 0 \\
\hline & b4 & 0 & 0.01481212 & 0 & 0 & 0 & 0 & 0 & 0 \\
\hline & b3 & -0.01369149 & 0.00089190 & 0 & 0 & 0 & 0 & 0 & 0 \\
\hline & b2 & 0.00543157 & -0.00003713 & 0 & 0 & 0 & 0 & 0 & 0 \\
\hline & b1 & 0.00117493 & 0.00000018 & 0.00052760 & 0.00034975 & 0.01515417 & -0.00307455 & 0.02016082 & 0.00450260 \\
\hline & b0 & 0.00001611 & -0.00000001 & -0.00001931 & -0.00000022 & -0.00061802 & -0.00041995 & -0.00082775 & -0.00022896 \\
\hline \multirow{6}{*}{$y_{10}$} & b5 & 0 & 0 & 0 & 0 & 0 & 0 & 0 & 0 \\
\hline & b4 & 0 & 0 & 0 & 0 & 0 & 0 & 0 & 0 \\
\hline & b3 & 0 & 0 & 0 & 0 & 0 & 0 & 0 & 0 \\
\hline & b2 & 0 & 0 & 0 & 0 & 0 & 0 & 0 & 0 \\
\hline & b1 & 0.01940940 & 0.04746217 & 0.00066295 & -0.00163601 & 0.01669626 & 0.02623611 & 0.01140498 & 0.01031737 \\
\hline & b0 & 0.00014096 & -0.00185563 & -0.00002042 & 0.00008011 & -0.00096968 & -0.00182250 & -0.00067438 & -0.00035287 \\
\hline \multirow{6}{*}{$y_{11}$} & b5 & 0 & 0 & 0 & 0 & 0 & 0 & 0 & 0 \\
\hline & b4 & 0 & -0.00687319 & 0 & 0 & 0 & 0 & 0 & 0 \\
\hline & b3 & -0.00668084 & 0.00186677 & 0 & 0 & 0 & 0 & 0 & 0 \\
\hline & b2 & 0.00224987 & -0.00008524 & 0 & 0 & 0 & 0 & 0 & 0 \\
\hline & b1 & 0.00042371 & -0.00000002 & 0.00005242 & -0.00032051 & -0.00083542 & -0.00114122 & -0.00063267 & 0.00011694 \\
\hline & b0 & 0.00000125 & 0 & -0.00000261 & 0.00001386 & -0.00000780 & -0.00000831 & -0.00000504 & -0.00001677 \\
\hline
\end{tabular}


Table 26 - Plant 4 - Transfer Function Denominator Parameters

\begin{tabular}{|c|c|c|c|c|c|c|c|c|c|}
\hline P4 & D & $u_{1}$ & $u_{2}$ & $u_{3}$ & $u_{4}$ & $u_{5}$ & $u_{6}$ & $u_{7}$ & $d_{l}$ \\
\hline \multirow{6}{*}{$y_{1}$} & a5 & 1 & 1 & 0 & 0 & 0 & 0 & 0 & 0 \\
\hline & a4 & 0.23296424 & 0.07863813 & 0 & 0 & 0 & 0 & 0 & 0 \\
\hline & a3 & 0.08208049 & 0.00430576 & 1 & 0 & 0 & 0 & 0 & 0 \\
\hline & $\mathrm{a} 2$ & 0.00758918 & 0.00005046 & 0.73141937 & 1 & 1 & 1 & 1 & 1 \\
\hline & a1 & 0.00027850 & 0.00000006 & 0.02366003 & 0.41143877 & 0.06512178 & 0.08150280 & 0.05821337 & 0.04183427 \\
\hline & $\mathrm{a} 0$ & 0.00000193 & 0 & 0.00016993 & 0.00508471 & 0.00064549 & 0.00082135 & 0.00056422 & 0.00038562 \\
\hline \multirow{6}{*}{$y_{2}$} & a5 & 1 & 0 & 0 & 0 & 0 & 0 & 0 & 0 \\
\hline & $\mathrm{a} 4$ & 0.23708439 & 0 & 0 & 0 & 0 & 0 & 0 & 0 \\
\hline & a3 & 0.06591165 & 1 & 0 & 0 & 0 & 0 & 0 & 0 \\
\hline & a2 & 0.00480229 & 0.05239921 & 1 & 1 & 1 & 1 & 1 & 1 \\
\hline & a1 & 0.00020995 & 0.00222654 & 0.03984005 & 1.27894468 & 0.10426372 & 0.20629258 & 0.08168182 & 0.05338634 \\
\hline & $\mathrm{a} 0$ & 0.00000144 & 0.00001732 & 0.00098598 & 0.01041943 & 0.00109114 & 0.00224910 & 0.00083210 & 0.00051304 \\
\hline \multirow{6}{*}{$y_{3}$} & a5 & 0 & 0 & 0 & 0 & 0 & 0 & 0 & 0 \\
\hline & a4 & 0 & 0 & 0 & 1 & 0 & 0 & 0 & 1 \\
\hline & a3 & 1 & 1 & 0 & 0.43818533 & 0 & 0 & 0 & 0.06735221 \\
\hline & $\mathrm{a} 2$ & 0.09688217 & 0.05125249 & 1 & 0.02483772 & 1 & 1 & 1 & 0.00416706 \\
\hline & a1 & 0.00555968 & 0.00273708 & 0.92474185 & 0.00141974 & 0.24707656 & 0.31464865 & 0.30851965 & 0.00010757 \\
\hline & $\mathrm{a} 0$ & 0.00003700 & 0.00003040 & 0.05252992 & 0.00001331 & 0.00481350 & 0.00663588 & 0.00583065 & 0.00000058 \\
\hline \multirow{6}{*}{$y_{4}$} & a5 & 0 & 0 & 0 & 0 & 0 & 0 & 0 & 0 \\
\hline & a4 & 1 & 0 & 0 & 0 & 0 & 0 & 0 & 0 \\
\hline & a3 & 0.11694337 & 1 & 0 & 0 & 0 & 0 & 0 & 0 \\
\hline & a2 & 0.00578770 & 0.05029504 & 1 & 1 & 1 & 1 & 1 & 1 \\
\hline & a1 & 0.00006439 & 0.00278323 & 1.22673630 & 0.50232417 & 0.10301513 & 0.14395772 & 0.09151188 & 0.07403663 \\
\hline & $\mathrm{a} 0$ & 0.00000016 & 0.00002836 & 0.02585690 & 0.00679653 & 0.00125117 & 0.00182626 & 0.00108903 & 0.00085042 \\
\hline \multirow{6}{*}{$y_{5}$} & a5 & 0 & 0 & 0 & 0 & 0 & 0 & 0 & 0 \\
\hline & a4 & 0 & 0 & 0 & 0 & 0 & 0 & 0 & 0 \\
\hline & a3 & 1 & 0 & 0 & 0 & 0 & 0 & 0 & 0 \\
\hline & $\mathrm{a} 2$ & 0.10104154 & 1 & 1 & 1 & 1 & 1 & 1 & 1 \\
\hline & a1 & 0.00379845 & 0.04898411 & 0.43152954 & 0.79996287 & 0.06432041 & 0.06504308 & 0.05700327 & 0.04468609 \\
\hline & $\mathrm{a} 0$ & 0.00002731 & 0.00043381 & 0.00587453 & 0.00937255 & 0.00053787 & 0.00059174 & 0.00047837 & 0.00037514 \\
\hline \multirow{6}{*}{$y_{6}$} & a5 & 1 & 0 & 0 & 0 & 0 & 0 & 0 & 0 \\
\hline & $\mathrm{a} 4$ & 0.21972097 & 0 & 0 & 0 & 0 & 0 & 0 & 1 \\
\hline & a3 & 0.06659150 & 1 & 0 & 0 & 0 & 0 & 0 & 0.05154411 \\
\hline & $\mathrm{a} 2$ & 0.00442000 & 0.05491795 & 1 & 1 & 1 & 1 & 1 & 0.01303801 \\
\hline & a1 & 0.00019950 & 0.00300581 & 0.08144899 & 0.73302066 & 0.24001571 & 0.30546798 & 0.32850680 & 0.00021094 \\
\hline & $\mathrm{a} 0$ & 0.00000132 & 0.00003921 & 0.00422347 & 0.01847968 & 0.00588219 & 0.00835938 & 0.00771513 & 0 \\
\hline \multirow{6}{*}{$y_{7}$} & a5 & 0 & 0 & 0 & 0 & 0 & 0 & 0 & 0 \\
\hline & $\mathrm{a} 4$ & 0 & 0 & 0 & 0 & 0 & 0 & 0 & 0 \\
\hline & a3 & 0 & 1 & 0 & 0 & 0 & 0 & 0 & 0 \\
\hline & $\mathrm{a} 2$ & 1 & 0.05272244 & 1 & 1 & 1 & 1 & 1 & 1 \\
\hline & a1 & 0.04281203 & 0.00313944 & 1.01082640 & 0.55796812 & 0.26007935 & 0.31059134 & 0.32064062 & 0.45341583 \\
\hline & $\mathrm{a} 0$ & 0.00024024 & 0.00003952 & 0.02169965 & 0.00805290 & 0.00431904 & 0.00543513 & 0.00522737 & 0.00718090 \\
\hline \multirow{6}{*}{$y_{8}$} & a5 & 0 & 0 & 0 & 0 & 0 & 0 & 0 & 0 \\
\hline & $\mathrm{a} 4$ & 0 & 0 & 0 & 0 & 0 & 0 & 0 & 0 \\
\hline & a3 & 1 & 0 & 0 & 0 & 0 & 0 & 0 & 0 \\
\hline & $\mathrm{a} 2$ & 0.12351264 & 1 & 1 & 1 & 1 & 1 & 1 & 1 \\
\hline & a1 & 0.00484536 & 0.05383596 & 0.02844148 & 0.06383918 & 0.04919364 & 0.08293772 & 0.05958454 & 0.04818289 \\
\hline & $\mathrm{a} 0$ & 0.00003158 & 0.00045160 & 0.00035422 & 0.00041686 & 0.00041126 & 0.00068875 & 0.00048580 & 0.00037573 \\
\hline \multirow{6}{*}{$y_{9}$} & a5 & 0 & 1 & 0 & 0 & 0 & 0 & 0 & 0 \\
\hline & $\mathrm{a} 4$ & 0 & 0.03660570 & 0 & 0 & 0 & 0 & 0 & 0 \\
\hline & a3 & 1 & 0.00286174 & 0 & 0 & 0 & 0 & 0 & 0 \\
\hline & $\mathrm{a} 2$ & 0.10169929 & 0.00002187 & 1 & 1 & 1 & 1 & 1 & 1 \\
\hline & a1 & 0.00410603 & 0.00000033 & 0.06022577 & 0.00394322 & 0.14853279 & 0.14177080 & 0.30637232 & 0.16425635 \\
\hline & $\mathrm{a} 0$ & 0.00001749 & 0 & 0.00049377 & 0 & 0.00102863 & 0.00074390 & 0.00197746 & 0.00093493 \\
\hline \multirow{6}{*}{$y_{10}$} & a5 & 0 & 0 & 0 & 0 & 0 & 0 & 0 & 0 \\
\hline & $\mathrm{a} 4$ & 0 & 0 & 0 & 0 & 0 & 0 & 0 & 0 \\
\hline & a3 & 0 & 0 & 0 & 0 & 0 & 0 & 0 & 0 \\
\hline & a2 & 1 & 1 & 1 & 1 & 1 & 1 & 1 & 1 \\
\hline & a1 & 0.03593174 & 0.08606306 & 0.03073281 & 0.12177017 & 0.19248980 & 0.29722990 & 0.18198729 & 0.14743131 \\
\hline & $\mathrm{a} 0$ & 0.00015891 & 0.00097574 & 0.00057639 & 0.00097667 & 0.00208356 & 0.00329687 & 0.00192737 & 0.00151117 \\
\hline \multirow{6}{*}{$y_{11}$} & a5 & 0 & 1 & 0 & 0 & 0 & 0 & 0 & 0 \\
\hline & $\mathrm{a} 4$ & 0 & 0.25190654 & 0 & 0 & 0 & 0 & 0 & 0 \\
\hline & a3 & 1 & 0.01188150 & 0 & 0 & 0 & 0 & 0 & 0 \\
\hline & $\mathrm{a} 2$ & 0.09248629 & 0.00035869 & 1 & 1 & 1 & 1 & 1 & 1 \\
\hline & a1 & 0.00401231 & 0.00000029 & 0.01313662 & 0.04725453 & 0.01729359 & 0.01863502 & 0.01702169 & 0.02135520 \\
\hline & $\mathrm{a} 0$ & 0.00001712 & 0 & 0.00068071 & 0.00179233 & 0.00017796 & 0.00016128 & 0.00015325 & 0.00075203 \\
\hline
\end{tabular}


Table 27 - Manipulated Variables Positive Uncertainty Parameters

\begin{tabular}{|c|ccccccccccc|}
\hline $\mathrm{B}_{u}^{+}$ & $\mathrm{y}_{1}$ & $\mathrm{y}_{2}$ & $\mathrm{y}_{3}$ & $\mathrm{y}_{4}$ & $\mathrm{y}_{5}$ & $\mathrm{y}_{6}$ & $\mathrm{y}_{7}$ & $\mathrm{y}_{8}$ & $\mathrm{y}_{9}$ & $\mathrm{y}_{10}$ & $\mathrm{y}_{11}$ \\
\hline $\mathrm{u}_{1}$ & 0.0192 & 0.0270 & 0.0618 & 0.0762 & 0.0797 & 0.0403 & 0.0721 & 0.0435 & 0.0217 & 0.0836 & 0.0745 \\
$\mathrm{u}_{2}$ & 0.1299 & 0.0150 & 0.0499 & 0.0379 & 0.0305 & 0.0371 & 0.0148 & 0.0039 & 0.0230 & 0.0182 & 0.0250 \\
$\mathrm{u}_{3}$ & 0.0060 & 0.0116 & 0.0098 & 0.0084 & 0.0181 & 0.0085 & 0.0129 & 0.0116 & 0.0254 & 0.0857 & 0.0973 \\
$\mathrm{u}_{4}$ & 0.0040 & 0.0235 & 0.0195 & 0.0181 & 0.0298 & 0.0049 & 0.0510 & 0.0458 & 0.0911 & 0.0773 & 0.0977 \\
$\mathrm{u}_{5}$ & 0.0384 & 0.0187 & 0.0304 & 0.0404 & 0.0738 & 0.0299 & 0.0259 & 0.0152 & 0.0472 & 0.0284 & 0.0572 \\
$\mathrm{u}_{6}$ & 0.0276 & 0.0169 & 0.0225 & 0.0372 & 0.0658 & 0.0273 & 0.0232 & 0.0822 & 0.0323 & 0.0264 & 0.0346 \\
$\mathrm{u}_{7}$ & 0.0162 & 0.0103 & 0.0025 & 0.0074 & 0.0404 & 0.0019 & 0.0178 & 0.1024 & 0.0304 & 0.0134 & 0.0119 \\
\hline
\end{tabular}

Table 28 - Disturbance Positive Uncertainty Parameters

\begin{tabular}{|c|ccccccccccc|}
\hline $\mathrm{B}_{d}^{+}$ & $\mathrm{y}_{1}$ & $\mathrm{y}_{2}$ & $\mathrm{y}_{3}$ & $\mathrm{y}_{4}$ & $\mathrm{y}_{5}$ & $\mathrm{y}_{6}$ & $\mathrm{y}_{7}$ & $\mathrm{y}_{8}$ & $\mathrm{y}_{9}$ & $\mathrm{y}_{10}$ & $\mathrm{y}_{11}$ \\
\hline $\mathrm{d}_{1}$ & 0.0301 & 0.0764 & 0.0091 & 0.0470 & 0.0266 & 0.0209 & 0.0391 & 0.0119 & 0.0151 & 0.0278 & 0.0838 \\
\hline
\end{tabular}

Table 29 - Manipulated Variables Positive Uncertainty Parameters

\begin{tabular}{|c|c|c|c|c|c|c|c|c|c|c|c|}
\hline $\mathrm{B}_{u}^{-}$ & $\mathrm{y}_{1}$ & $\mathrm{y}_{2}$ & $\mathrm{y}_{3}$ & $\mathrm{y}_{4}$ & $\mathrm{y}_{5}$ & $\mathrm{y}_{6}$ & $y_{7}$ & $\mathrm{y}_{8}$ & $\mathrm{y}_{9}$ & $\mathrm{y}_{10}$ & $\mathrm{y}_{11}$ \\
\hline & -0.0934 & -0.1023 & -0.0433 & -0.0881 & -0.0659 & -0.0604 & -0.0128 & -0.0040 & -0.2949 & -0.0166 & -0.0724 \\
\hline & -0.1072 & -0.0035 & -0.0312 & -0.0202 & -0.0054 & -0.0179 & -0.0159 & -0.0096 & -0.0058 & -0.0161 & -0.0003 \\
\hline & -0.0057 & -0.0137 & -0.0096 & -0.0088 & -0.0194 & -0.0043 & -0.0082 & -0.0031 & -0.0694 & -0.1224 & -0.0288 \\
\hline & -0.0168 & -0.0139 & -0.0219 & -0.0106 & -0.0312 & -0.0023 & -0.0398 & -0.1082 & -0.0435 & -0.0476 & -0.2217 \\
\hline & -0.0665 & -0.0543 & -0.0352 & -0.0301 & -0.0323 & -0.0307 & -0.0366 & -0.0493 & -0.0777 & -0.0388 & -0.0217 \\
\hline & -0.0511 & -0.0477 & -0.0375 & -0.0345 & -0.0216 & -0.0289 & -0.0469 & -0.0278 & -0.1124 & -0.0466 & -0.0258 \\
\hline & -0.0166 & -0.0177 & -0.0060 & -0.0070 & -0.0252 & -0.0036 & -0.0369 & -0.0375 & -0.0565 & -0.0392 & -0.0139 \\
\hline
\end{tabular}

Table 30 - Disturbance Negative Uncertainty Parameters

\begin{tabular}{|c|ccccccccccc|}
\hline $\mathrm{B}_{d}^{-}$ & $\mathrm{y}_{1}$ & $\mathrm{y}_{2}$ & $\mathrm{y}_{3}$ & $\mathrm{y}_{4}$ & $\mathrm{y}_{5}$ & $\mathrm{y}_{6}$ & $\mathrm{y}_{7}$ & $\mathrm{y}_{8}$ & $\mathrm{y}_{9}$ & $\mathrm{y}_{10}$ & $\mathrm{y}_{11}$ \\
\hline $\mathrm{d}_{1}$ & -0.0982 & -0.0788 & -0.0688 & -0.0885 & -0.0830 & -0.0377 & -0.0438 & -0.0406 & -0.0421 & -0.0468 & -0.0440 \\
\hline
\end{tabular}

